\title{
The unified theory of repression
}

\author{
Matthew Hugh Erdelyi \\ Department of Psychology, Brooklyn College and the Graduate School, \\ The City University of New York, Brooklyn, NY 11210-2889 \\ iyledre@patmedia.net
}

\begin{abstract}
Repression has become an empirical fact that is at once obvious and problematic. Fragmented clinical and laboratory traditions and disputed terminology have resulted in a Babel of misunderstandings in which false distinctions are imposed (e.g., between repression and suppression) and necessary distinctions not drawn (e.g., between the mechanism and the use to which it is put, defense being just one). "Repression" was introduced by Herbart to designate the (nondefensive) inhibition of ideas by other ideas in their struggle for consciousness. Freud adapted repression to the defensive inhibition of "unbearable" mental contents. Substantial experimental literatures on attentional biases, thought avoidance, interference, and intentional forgetting exist, the oldest prototype being the work of Ebbinghaus, who showed that intentional avoidance of memories results in their progressive forgetting over time. It has now become clear, as clinicians had claimed, that the inaccessible materials are often available and emerge indirectly (e.g., procedurally, implicitly). It is also now established that the Ebbinghaus retention function can be partly reversed, with resulting increases of conscious memory over time (hypermnesia). Freud's clinical experience revealed early on that exclusion from consciousness was effected not just by simple repression (inhibition) but also by a variety of distorting techniques, some deployed to degrade latent contents (denial), all eventually subsumed under the rubric of defense mechanisms ("repression in the widest sense"). Freudian and Bartlettian distortions are essentially the same, even in name, except for motive (cognitive vs. emotional), and experimentally induced false memories and other "memory illusions" are laboratory analogs of self-induced distortions.
\end{abstract}

Keywords: avoidance; Bartlett; defense; denial; distortion; Ebbinghaus; false-memories; Freud; inhibition; repression; suppression

\section{Introduction}

Repression has been a puzzle for scientific psychology. It is not clear to many, even at this date, whether repression is best regarded as an obvious fact of mental life or an outright (and even dangerous) myth. In this article I sketch out a theory of repression that integrates the largely dissociated data of the clinic and the laboratory into a unified framework that is simple, rich - and right.

The article is organized into four sections besides this Introduction. First, in a historical analysis (sect. 2), I show that the classic conception of repression, from Herbart to Freud, is consistent with modern laboratory research, but that confusion has resulted from a semantic distortion introduced, ironically, by Anna Freud, who insisted that repression needed to be an unconscious process, its conscious counterpart being "suppression." Sigmund Freud, actually, used repression and suppression interchangeably and insisted on "the unity of mental life" across the conscious-unconscious continuum, so that "repression" could be both conscious and unconscious. The historical analysis is thought to be important because it dissolves much of the controversy surrounding repression. Building on this historical foundation, the third section articulates the unified theory of repression that is being proposed. Repression, conceived of as a class of consciousness-lowering processes, is divided into two subclasses, inhibitory and elaborative processes. Inhibitory (or simple) repression involves cognitive avoidance (notthinking) of some target material and leads to loss of accessible memory. Some of the lost memory may, however, express itself indirectly and may be partially recovered with subsequent retrieval effort. Elaborative repression distorts the original memory through a variety of transformations and false additions. These two subclasses of memory degradation subsume most of the classic clinical manifestations of repression and, critically, are extensively buttressed, as shown in Sections 4 (on inhibition) and 5 (on elaborative distortions), by the experimental literature. Consequently, a viable unified framework for repression is afforded.

\section{History and definition of repression, including distortions of the concept}

Although traditionally associated with Freud, the term as well as basic concept of repression was introduced into psychology more than half a century before the advent of psychoanalysis by one of the founders of scientific

Matthew Erdelyi was born in Budapest, Hungary, lived in Venezuela for several years, and finally settled in the United States, where he obtained his Ph.D. at Yale. He speaks no language without an accent. He is Professor of Psychology and formerly the Stern Professor of Humor at Brooklyn College, City University of New York. Erdelyi has pursued experimental and theoretical work on subliminal perception, the recovery of inaccessible memories, and psychodynamics. Along with many articles, he has published two books, Psychoanalysis: Freud's Cognitive Psychology (1985) and The Recovery of Unconscious Memories: Hypermnesia and Reminiscence (1996). He has been a visiting scholar at various universities and was a Fellow at the Center for Advanced Study in the Behavioral Sciences in Stanford. 
psychology, Johann Herbart (1824-1825), to designate the inhibition of ideas by other ideas. Herbart's "repression" was not a defensive repression. It is important to distinguish between the mechanism and the defense (Anderson \& Green 2001; Erdelyi 1993). The mechanism is the basic process; the defense is the use to which the mechanism is put - and there could be many uses other than defense. For Herbart, repression was a necessary consequence of the limited capacity of consciousness. By being aware of one idea, we necessarily foreclose being aware of another idea. According to Herbart, the inhibited ideas do not cease to exist but pass into a "state of tendency" - the philosophically correct term of those days for "unconscious" - and fall below the "threshold of consciousness." The repressed ideas can, with changed circumstances or in recombinations, overthrow the ideas currently in consciousness and repress them in turn.

Freud, who was influenced by Herbart, at least indirectly (see Sand 1988), also maintained that repressed ideas do not cease to exist. Later in this article I examine some experimental data that bear on this controversial assumption. Even before the creation of psychoanalysis, while he was still tinkering with hypnotherapy and catharsis, Freud already espoused the notion of defensive repression (though "repression" was not the first term he used), hewing to a model of mind reminiscent of Herbart's dynamic model of consciousness. Freud parted with the reigning philosophical assumption of his day that the mind was coterminous with consciousness - as we know, consciousness became for Freud, as it has now become for scientific psychology, a mere tip of the psychological iceberg - and conceived of the conflict unfolding on a deeper plane: Psychological subsystems, not all accessible to consciousness, were in conflict with each other and tried to inhibit the operation and accessibility of the other.

Some years before The Interpretation of Dreams (Freud $1900 / 1953$ ), which for many marks the beginning of psychoanalysis, Freud (1892-1893/1966) published a two-part case history of a patient who was having problems breast-feeding her baby. Freud sketched out a dynamic formulation of the woman's problem. She was conceived to be in conflict - without being aware of the conflict: She both wished and did not wish to breast-feed her child. Because awareness of her reluctance would have been painful to her, she "inhibited the antithetic idea" (the reluctance to breast-feed) and "dissociated" it from her awareness. The antithetic idea does not thereby get abolished but "establishes itself, so to speak, as a 'counter-will', while the patient is aware with astonishment of having a will which is resolute but powerless" (p. 122).

The terms dissociated and inhibited were soon joined by repression and suppression in Breuer and Freud's (1893/ 1955) "Preliminary Communications," which became the first section of their 1895 classic, Studies on Hysteria (in my view, the effective starting point of psychoanalysis). In this work Freud and Breuer write:

It was a question of things which the patient wished to forget, and therefore intentionally repressed from his conscious thought and inhibited and suppressed. (Breuer \& Freud $1895 / 1955$, p. 10)

Some 20 years later, in his article "Repression," Freud (1915a/1957/1963) provided a straightforward definition of repression: "the essence of repression lies simply in the function of rejecting and keeping something out of consciousness" (p. 147, emphasis in the original).

It should be noted that there is no mention in either the 1895 formulation or the 1915 definition that repression - the process - is itself unconscious. Yet, introductory texts in psychology or psychoanalysis treat as axiomatic that the defenses, including repression, are unconscious processes. The conscious counterpart of repression, it is averred, is "suppression." Yet, as I have been suggesting for some time (e.g., Erdelyi 1990; 1993; 2001a), this rendering of repression as a necessarily unconscious process, and its distinction from suppression, constitutes a "grand-Bartlett effect" - a reconstructive memory distortion operating at the level of the field. Although Freud's half-century of psychological writing on repression is not without some ambiguities and even contradictions, the overwhelming textual evidence is that Freud used repression and suppression interchangeably, from his earliest writings (e.g., 1893, as we have seen) to his last (e.g., An Outline of Psycho-Analysis, Freud 1940/1964).

It was Anna Freud (1936/1937), trying to tidy up her father's often messy work, who introduced in her book, The Ego and the Mechanisms of Defence, the diktat that repression was unconscious and suppression was its conscious counterpart. This was, by the way, a retrograde tack, since her father had pursued a Gordian knotcutting strategy of insisting on the continuity of mental life, whether for "thought," "resistance," "guilt," or other mental processes. Thus, "it would be unjustifiable and inexpedient to make a break in the unity of mental life for the sake of propping up a definition" (Sigmund Freud 1938/1964, p. 286). Complex mental processes could be conscious or unconscious, according to Freud père; they did not become something else just because they crossed some hypothetical threshold of consciousness. (For the view that repression can be conscious or unconscious, see also Brewin [2003] and McNally [2003]).

I make a fuss about this semantic point because if the unconscious-rendering process, repression, can be both conscious and unconscious, then there is no controversy. If repression $=$ suppression, then everybody believes in repression. The myth morphs into the obvious.

Let us return to Sigmund Freud's first use of "repression" (Breuer \& Freud 1895/1955, p. 10). Sensing a problem here, the later psychoanalytic establishment deployed the following explanatory footnote:

This is the first appearance of the term "repressed" ('verdrängt') in what is to be its psycho-analytic sense.... The word "intentionally" merely indicates the existence of a motive and carries no implication of conscious intention. (Footnote 1, in Breuer \& Freud [1895/1955], Studies on Hysteria, p. 10)

Although I have left out some of the arguments in the long, full footnote, it is hard to reconcile with the case studies making up the Studies on Hysteria. The patients discussed are often deliberately and consciously pushing out some memory or thought from consciousness. Indeed, at one point, Freud uses the expression, "conscious rejection" (p. 134). In his already-cited article "Repression," Freud (1915a/1957/1963) speaks of the "censorship of the conscious" (pp. 149-150) and actually feels obliged to warn the reader against assuming that repression is always a conscious undertaking: "it is a mistake to emphasize only 
the repulsion which operates from the direction of the conscious upon what is to be repressed" (p. 148). In the Case of the Psychotic Dr. Schreber, Freud states that repression "emanates from the highly developed systems of the ego - systems which are capable of being conscious" (Freud 1911/1958, p. 67). In the Case of the "Rat Man" (Freud 1909/1955), thought-avoidance is effected by conscious thought-distraction: "His original intention ... had been repressed by his praying" (p. 193). In a footnote added in 1914 to The Interpretation of Dreams, Freud remarks, "In any account of the theory of repression it would have to be laid down that a thought becomes repressed as a result of the continued influence upon it of two factors: It is pushed from the one side (by the censorship of Cs.) and from the other (by the Ucs.)" (1900/1953, p. 547). In his article, "The Unconscious," Freud (1915b/1957) states, "Consciousness stands in no simple relation ... to repression (p. 192).

There are occasional materials tending to point in the opposite direction. For example, in an 1896 paper, Freud places at one point a parenthetical "unconscious" in front of the term "defense" - "(unconscious) defence" (Freud 1896a/1962). In The Interpretation of Dreams (1900/1953), Freud suggests that repression "lays more stress" than suppression "upon the fact of attachment to the unconscious" (p. 606). But such materials are isolated and even ambiguous tidbits in a massive corpus.

In psychiatry and clinical psychology of the last two decades, the conscious-unconscious issue in repression and defense has played out in contradictory trends. In the DSM-III-R (American Psychiatric Association 1987), the treatment of the material is in the revisionist vein. Defense, which had been conceived of by Freud as a voluntary ("intentional," "motivated") act, is rendered as "relatively involuntary" (p. 393). In a formulation that touches neither on the question of intention nor the conscious or unconscious nature of the process, repression is defined as "a mechanism in which the person is unable to remember or be cognitively aware of disturbing wishes, feelings, thoughts, or experiences" (p. 394). Completing the revisionist arc, Phebe Cramer (1998; 2000) disallows both consciousness and intentionality in defense mechanisms; when they are present, the processes are recast - and forcefully distinguished from defense mechanisms - as coping mechanisms. The DSM-IV-TR (American Psychiatric Association 2000) significantly backtracks, however: Defense mechanisms and "coping styles" are treated as equivalent (p. 807), and defense mechanisms are described as processes of which "individuals are often unaware (p. 807, emphasis added), even if it is suggested, somewhat self-contradictorily, that "Defense mechanisms (or coping styles) are automatic psychological processes" (p. 807). It is no longer clear what, if any, substantive distinction is retained between repression ("expelling disturbing wishes, thoughts, or experiences from conscious awareness") and suppression ("intentionally avoiding thinking about disturbing problems" [p. 813]). Recently, George Vaillant (1998) has urged "a way to circumvent the false dichotomy of conscious/unconscious" (p. 1156).

In a strict psychophysical sense, true subliminality is problematical because there may be no true limen (see Erdelyi 1986; 2004a; 2004b; Macmillan 1986; Merikle \&
Reingold 1991) and therefore the conscious-unconscious distinction may not be categorical but polar, representing opposite ends on a grainy continuum - much like the distinctions between child and adult, day and night, perception and memory (Erdelyi 1986; 1996; 2004a; 2004b). Freud himself, in his later writings, began to sense this problem. An inspection of his 1923 and 1933 diagrams of the structural model (Freud 1923/1961, p. 24; 1933/ 1964 , p. 78) suggests that there is no limen or threshold separating consciousness from unconsciousness, but a fuzzy region in which the two merge. Thus, even if one sought it, a crisp, categorical distinction between suppression and repression may be unrealizable. ${ }^{1}$ Laboratory evidence shows (see sect. 4) that conscious memory waxes and wanes over time. Consciousness for the past, therefore, is not either-or but an integral (as in calculus) over time, varying with the time at which testing begins and the temporal window over which it is evaluated (Erdelyi 1996; 2004a; 2004b; Ionescu \& Erdelyi 1992).

It is noteworthy that none of the experiments in the laboratory literature evaluated by David Holmes (1974; 1990) in his influential reviews, which caused him to suggest the abandonment of repression for want of laboratory confirmation, ever sought to establish that the variable investigated (repression) was unconscious. Thus, none of the evaluated experiments satisfied Holmes's own criterion for repression. It should be noted also that the trait approach to repression, in which "repressiveness" is assessed by personality tests (e.g., Byrne 1961; Weinberger 1990; Weinberger et al. 1979), cannot establish whether the putative repressors are doing their repressing unconsciously. Not surprisingly, in a recent review of defense mechanism measures, Davidson and MacGregor (1998) found that no instrument succeeded in establishing the unconscious criterion for defense mechanisms (see their Table 1, p. 984).

In recent years, the idea of repression as inhibition or suppression of selected mental contents has increasingly moved into the mainstream of psychology. An important development in the experimental literature on memory has been the recognition of "retrieval inhibition" as a "missing link" in intentional forgetting (Bjork 1989; Geiselman et al. 1983). The influence of neuroscience has pushed the field past pat computer models to more realistic biological conceptualizations and has led to an appreciation of the evolution of the brain yielding inhibition by higher-order regions of more primitive ones (e.g., Bjork 1989). In a recent book on repression, Michael Billig (1999) suggests that normal language development involves the mastering and automatization of skills for both expressing and repressing ideas. Evolutionary psychology (e.g., Badcock 2000; Wright 1994) has pointed to the importance of repression - and self-deception in general - for various adaptive purposes, including the more effective deception of others. Anderson and his colleagues (e.g., Anderson 2001; Anderson \& Green 2001; Levy \& Anderson 2002) have experimentally underscored the "adaptive" function of retrieval inhibition for the purpose of avoiding potentially interfering or otherwise unwanted memories. In recent neuroscience research, Anderson and colleagues (Anderson et al. 2004) identify a neurological circuit involved in intentional retrieval-inhibition and consequent forgetting: Activation of prefrontal cortical areas (associated with "executive 
control functions") results in the inhibition of hippocampal activity, with resultant declines in accessible memory. Some convergence is thus in evidence.

A final task remains in this historical overview: to differentiate between Freud's repression in the narrow sense and repression in the general sense. In Freud's earliest writings, he meant by repression the simple inhibition or exclusion from consciousness of some idea or impulse or memory. Almost immediately, as his clinical experience forced him to be more nuanced about his material, he noticed that the process of repression could be partial. In 1894, for example, he noted the phenomenon of isolation (or intellectualization): Certain patients seemed to dissociate the factual and affective components of an idea and repress only the affective part. Such individuals could evidence endless conscious knowledge of the facts and yet be deeply out of touch with the emotional aspects of these facts. (Laboratory psychology, in its experimental assays, has ignored such variations on the basic process.) With further clinical experience, Freud was able to identify a whole basketful of such partial repressions, including, projection, reaction formation, displacement, symbolization, rationalization, and even representation by triviality. At first, Freud treated all these inhibitions or distortions of reality as "repression," but by 1926, he felt compelled to simplify for fear of losing his original simple concept of repression (inhibition, suppression, etc.). And so he made a distinction between repression in the narrow (original) sense and "repression in the widest sense" (Freud 1937/1964, p. 257), which subsumed all the defensive mechanisms, including repression in the narrow sense, and renamed the whole set defense processes or defense mechanisms. (For example: "It is of advantage to distinguish the more general notion of 'defence' from 'repression.' Repression is only one of the mechanisms which defence makes use of" [Freud 1926a/1959, p. 114].)

\section{The unified theory of repression}

The theory is unified in that it integrates largely dissociated traditions of the clinic and laboratory. It builds substantially on the just-reviewed work of Sigmund Freud (with some emendations) and harmonizes this material with well-established experimental knowledge derived from the psychological laboratory and, to some extent, from neuroscience.

\subsection{Conceptualization of repression}

Repression, essentially, is a consciousness-lowering process. It consists of a class of operations that reduces the accessibility to consciousness of some target material. Repression, further, is instrumental in bringing about a discernible goal - for example, preventing the span of consciousness from being breached or preventing some intolerable psychological material from entering consciousness.

The null hypothesis is adopted that repression is the same mechanism whether it is used for defense or some other purpose. Only when the goal of repression is to defend against psychological distress is the mechanism of repression conceived of as a mechanism of defense (see also Erdelyi 1990; 1993). Thus, the mechanism of repression can be probed in the laboratory irrespective of its goal.

In the unified theory, repression is divided into two subclasses: (1) inhibitory or subtractive processes (e.g., degrading the "signal"), and (2) elaborative or additive processes (e.g., adding "noise" to the signal). This division aligns with the psychological development of the concept both in Freudian and experimental psychology. As noted in the preceding historical review, Freud's initial conception of "repression" was of the inhibitory or subtractive variety ("suppression," "inhibition," "dissociation") followed in short order by a number of elaborative "transformations" ("constructions," "distortions"), among them, rationalization, projection, reversal, displacement, symbolization. Experimental psychology, similarly, began with subtractive processes - for example, cognitive avoidance that results in forgetting (Ebbinghaus) - followed gradually, as the field began to deal with more complex stimuli (short stories instead of lists of nonsense syllables or words), with constructive/reconstructive processes (e.g., Bartlett). This two-part division of repression is easily accommodated by Bartlett since one of his basic "reconstructions" is omission (e.g., Bartlett 1932, p. 66, pp. 99-102, pp. 125-126), with the other operations comprising standard elaborative reconstructions (see sect. 5.1). ${ }^{2}$

\subsection{Inhibitory or subtractive processes}

In inhibitory repression the consciousness-lowering operation is readily conceived of as some type of psychological subtraction that results in lower consciousness (e.g., we subtract attentional allocation from a channel, we reduce or eliminate thinking about some material).

3.2.1. Simple inhibition. In simple inhibition, about which a huge amount of research literature has accumulated (see sect. 4), some specific target (a list of nonsense syllables, some dreadful event, a taboo desire) is inhibited or subtracted out from consciousness. The classic clinical literature, dissociated from laboratory research for most of its history, did not assimilate the Ebbinghausian tradition and conflated the inhibitory operation with the resulting effects on memory. In the present work, the two are separated: Inhibition of consciousness for some target material results, over time, in the progressive degradation of accessible memory for the target material. Thus, remove the inhibition, and the material - at least some of it - will have been forgotten. Contrary to Ebbinghaus, however, forgetting does not imply decay - permanent loss - of the original material. With retrieval effort ("retrieval time" [Roediger \& Thorpe 1978]; "the work of remembering" [e.g., Breuer \& Freud 1895/1955]), the forgotten partially returns: With retrieval effort, memory is hypermnesic (enhanced), just as it is amnesic (diminished) with retrieval inhibition. Thus, Ebbinghaus represents only one side of what happens to memory over time.

Here is a clinical fragment, The Case of B., which illustrates concretely some of the foregoing proposals and which points to several additional considerations that bear on inhibitory repression:

B., a young man who sought therapy for a pathological grief reaction to the death of his brother five years earlier, noted in his intake interview that he avoids thinking of the traumatic 
event. (For example: "I clearly avoided to know anything; everything I know bothers me" [1st Evaluation Interview]; "I couldn't metabolize-digest the event. It makes me still sad - don't feel comfortable discussing" [1st Therapy Session]; "I don't think about many details of things that happened. I don't remember too many different situations about him, I just remember him ... like smoke .... It's blurry, it's not a very clear view" [3rd Therapy Session]. But, by the fourth therapy session, B. states: "I am [now] thinking more [of my brother] - I remember him more."

For years, B. had avoided potential reminders of his brother's death. He could not bear to attend the funeral and later, to avoid the dreaded topic, would lie to acquaintances who inquired after the brother, leading them to believe that the brother was alive. The whole-scale repression of the brother complex was not altogether successful. The repressed brother returned to haunt him in a variety of ways. Past the midpoint of his therapy, when he was complaining of sexual problems, B. reported that the image of his brother's face appeared to him as he was attempting intercourse with his (B.'s) girlfriend. (Adapted from Erdelyi 1993, pp. 137-138, with permission from Prentice Hall)

Although there is no independent evaluation of B.'s ongoing recall level for his brother up to and through his therapy, it is noteworthy that the alleged amnesia and subsequent hypermnesia resemble the effects obtained in the laboratory (see sect. 4): Thought-avoidance yields amnesia and the subsequent refocusing of retrieval effort, hypermnesia. Also worth underscoring is that the material being avoided by $\mathrm{B}$. was a major emotional event and not some innocuous laboratory stimulus. Finally, B. seemed to be conscious of his efforts to avoid thinking of his dead brother.

I now consider some nuances regarding the interpretation of empirical data on defensive inhibition.

\subsubsection{Inaccessibility associated with emotionality need not} imply defense. Consider the experiment on "cognitive masking” by Erdelyi and Appelbaum (1973). Observant Jewish students (pilot work had shown weak effects with mixed groups of students) were flashed a slide with a swastika or a window (the swastika with arms extended at right angles to form a square framing perpendicular cross-bars) in the center, and neutral symbols circularly arrayed around the central element. No sensitivity $\left(d^{\prime}\right)$ differences were found for the swastika versus the window, but there was a $d^{\prime}$ diminution for the surround items arrayed around the swastika. Was this a case of perceptual defense/repression not being powerful enough to stave off the perception of the center swastika, but sufficient to defend against its peripheral surround? There was a third, identically treated group that was flashed a stimulus with a different central item, a Star of David, which also, like the swastika center, produced a diminution of $d^{\prime}$ for the surround items. On the assumption that the Jewish students did not have a propensity to defend against a Star of David, the conclusion reached was that attention had been captured by either of the emotionally significant center items (swastika or Star of David), resulting in diminished processing resources for the surround items. This type of phenomenon has been persistently observed in the experimental literature, under various labels (e.g., "narrowing of attention," "weapon focus," "mental shock"): Central items are well processed, but the peripheral items are poorly perceived or recalled (see Easterbrook 1959; Loftus \&
Burns 1982; Schooler \& Eich 2000). The amnesia that results from cognitive masking can be retrograde (Loftus \& Burns 1982) or anterograde (Erdelyi \& Blumenthal 1973) - or it may be amnesia for contemporaneous details (as in Erdelyi \& Appelbaum 1973). The relevance of cognitive masking for any putative defense involving decrements in performance with emotional stimuli is that diminished performance may be due to nondefensive allocation of processing resources to other items or channels. Thus, it cannot be automatically assumed that poorer performance in connection with emotional stimuli is a consequence of defensive repression. For this reason it is particularly convenient to have clinical cases (like the Case of B.) in which the patient explicitly asserts a conscious effort to avoid thinking about the disturbing material because of the distress that the material would provoke.

The phenomenon of cognitive masking has significant implications for the contentious area of memory for trauma (for far-ranging overviews, see Brewin 2003; Brown et al. 1998; Brown et al. 1999; Freyd 1996; Kihlstrom 2006; McNally 2003; Pope et al. 1998; Terr 1993; van der Kolk et al. 1996). Does emotionality enhance or degrade memory? The answer, actually, seems to be yes and yes. There is widespread consensus that emotionality enhances memory for central elements of stimuli but degrades memory for peripheral items. This simple formulation is readily linked to laboratory studies where the stimulus is relatively simple. In the Erdelyi and Appelbaum (1973) experiment, for example, there is, literally, a central item (e.g., a swastika) and an array of items surrounding the central item. In real-life settings, where the "stimulus" may last minutes, hours, days, and even years, it is not so obvious what is "central" and what is "peripheral," except by post-hoc definition. The simple central-versus-peripheral distinction, in any case, yields an ambiguous answer for overall memory, which would be made up of central and peripheral items (however defined). Since, as the current consensus holds, emotionality has opposite effects on central and peripheral items, it is not difficult to conceive of different experiments yielding different conclusions about overall memory for emotional stimuli on the basis of different framings of what constitutes "central" and "peripheral" components.

Perhaps a more basic question is what constitutes "memory," which it would be a mistake to conceive of as some homogenous mass about which a sweeping generalization is possible. Memory, in many significant respects, is a heterogeneous construct, and what might apply to one aspect of memory might not apply to another (see, particularly, Brewin 2003; Erdelyi 1990; 1996; Schooler \& Eich 2000; van der Kolk 1994; van der Kolk et al. 1996). Consider the Case of B. Let us assume, for the sake of argument, that what B. states is roughly accurate and that his memory for his dead brother is "blurry," "not ... very clear," "like smoke." Is B. actually suffering psychogenic amnesia for his brother - or the opposite, hypermnesia? The answer, as already suggested, is both. For some five years, B. has avoided thinking, talking, or being reminded of his brother. Not surprisingly, his memory for his brother has become sketchy, though, of course, he has not outrightly forgotten his brother. Yet, in significant respects, B.'s problem, as in posttraumatic stress disorder 
(PTSD) (or kindred disorders from the past, such as "traumatic hysteria," or "neurosis"), is that B. remembers abnormally too much. After five years, most of us would have moved past our bereavement for a dead brother, along with its memorial entanglements. Such "fixation" on the past, the failure to move on, is what essentially defined neurosis for Freud. Similarly, for Pierre Janet (1889), "fixed ideas" played out as hysterical phenomena.

3.2.1.2. Temporal dynamics. One phenomenon that is not in dispute in the disputatious literature on traumatic memory is that patients vigorously try to avoid thinking of traumatic materials (e.g., Brewin 2003; Horowitz 1986; McNally 2003; DSM-IV 2000). What is not sufficiently emphasized, however, is that this effort need not immediately succeed. The effort at repression may eventually yield the desired result, but it may take time - days, weeks, or months - and so success at inhibitory repression may vary over time. It is also possible that the protracted effort at repression fails - there is no guarantee that any defense will work - and the patient may never succeed at inhibiting the traumatic material. Further, even if success at inhibitory repression is eventually achieved, resulting in diminution of accessible memory over time, there is no guarantee that at a later juncture there might not be a "return of the repressed" (e.g., Freud 1900/ 1953; 1917/1961/1963) or a "spontaneous recovery" (Pavlov 1927) of some of the material (see also Schacter 1996).

Thus, it may prove critical to consider temporal dynamics in responses to emotional stimuli because time may obscure shifts from hypermnesia to amnesia. Failure of repression at Time 1 may no longer be a failure at Time 2 - or vice versa. The possibility of response pattern reversals over time has only recently begun to be probed (e.g., Calvo \& Eysenck 2000; Erdelyi 2004a; 2004b; Mogg \& Bradley 1998; Shane \& Peterson 2004a).

3.2.1.3. Individual and stimulus differences. A serious flaw in laboratory efforts to demonstrate repression is that they fail to take individual differences into account. As noted already, a few trait measures of "repressiveness" have been introduced that, however, depend on arguable assumptions (e.g., that repressiveness is indexed by the intersection of low anxiety and high social desirability). What would be germane for the present treatment of repression is a straightforward test of ability to repress (not-think) about some target-material, irrespective of reason. Pilot work in my laboratory, in which subjects were asked to avoid thinking or free-associating about a provocative stimulus, suggests, as shown by on-line freeassociative fantasy, that subjects differ substantially in the extent to which - and for how long - they can maintain inhibition for the target material (e.g., Moore 2004). Further, as already intimated, there are likely to be substantial individual differences in the techniques of repression favored by different individuals. Thus, experiments using only one type of inhibition or distortion may lack content validity.

Further still, different stimulus materials are not equally likely to trigger defensive repression. On the one hand, the innocuous stimuli of the laboratory may not be sufficient for the purpose and may yield only cognitive masking effects. Traumatic events in the real world, on the other hand, may overwhelm efforts at defensive repression. Finally, stimulus intensity cannot be considered a simple dimension. Different traumatic stimuli - for example, a car accident versus a rape, or a rape by a stranger versus a rape by a caretaker (Freyd 1996) - may have quite different impacts. In sum, a serious effort at scientifically probing defenses requires a more complex approach than has been typical heretofore.

3.2.2. Structured inhibition. Just as a fig leaf is not plastered randomly on the body of a censored nude, defensive repression may be deployed in a nuanced, meaningfully structured way to subtract-out only the most problematic elements of an overall target. Isolation (or intellectualization), in which a person dissociates - or "isolates" - the factual aspects of an emotional memory from its emotional aspects and inhibits the emotional aspects, is one example of structured inhibition, and it has already been briefly mentioned.

In this section, I focus on denial, which is a ubiquitous form of structured repression - and is arguably the most neglected phenomenon of repression in experimental psychology. Denial has been defined in a number of different ways, some overlapping, some conflicting (e.g., Brenner 1973; A. Freud 1936/1937). The present treatment follows the conceptualization of denial as a defensive failure of insight (Erdelyi 1985). This conceptualization is broadly consistent with mainstream clinical use as well as everyday parlance (e.g., being "in denial"). The individual in denial does not fail visually to "see" some event but fails to "see" the deeper meaning or significance of the event.

A formalization of denial can be realized by making the classic Freudian distinction between the manifest (surface) and latent (deep) semantic content of stimuli. Experimental psychology often treats this vertical type of dissociation as suspect, probably because examples are usually taken from the realm of dreams or symptoms, which often yield disagreements about the interpreted latent contents. A methodologically more fruitful venue is the study of jokes (Erdelyi 1985; 1999), to which Freud $(1905 / 1958)$ devoted a whole book to show how jokes essentially captured the proposals he had set forth for dreams a few years earlier (Freud 1900/1953). In the case of jokes, it is obvious - to anyone who "gets" a joke - that below the surface meaning resides a different meaning. Without interpretation of the latent content there is no "getting" a joke - the interpretation is the getting. The interpretation of latent contents is not chancy. Subjects claiming to get a joke usually come up with the same interpretation. Application of signal detection theory (SDT) techniques (ROC curves, $d^{\prime}$ ) to measuring the sensitivity of subjects to latent contents of jokes reveals substantial individual differences (with split-half reliabilities easily exceeding $r=.60$ ) (Bergstein 2006; Bergstein \& Erdelyi, in preparation; Nishioka \& Kozbelt, in preparation). Thus, the interpretation of latent contents can be reliable and valid, even if some of us are more sensitive to latent contents than others (see also, Erdelyi 2001b).

Freud realized early on that context was critical for interpretation. For example: "dream-symbols ... frequently have more than one or even several meanings, and, as with Chinese script, the correct interpretation 
can only be arrived at on each occasion from the context" (Freud 1900/1953, p. 531); "mistakes have meaning [which] can be guessed or confirmed from the attendant circumstance" (Freud 1917/1961/1963, p. 51); "representation by the opposite," as in "ironic jokes" or sarcasm, which can often pose the "danger of being misunderstood," yields its "true meaning" by the speaker's "tone of voice" or by "some accompanying gesture" (Freud $1905 / 1958$, p. 174); and so on. The basic idea seems straightforward enough.

Even so, ambiguities and semantic drifts pervade the subject, both in the current literature (see, e.g., the variegated commentaries on Malcolm Macmillan's [2001] article on interpretation) and in Freud's own writings. Freud routinely defines the dream work as the set of techniques by which the latent content is transformed into the manifest content (e.g., Freud 1900/1953; 1917/ 1961/1963). In his book on jokes, Freud (1905/1958) demonstrates that jokes and dreams employ the "same psychical processes" (p. 88) and that "the joke work is doing the same thing as the dream work," namely (in the case of "tendentious jokes," involving sex or hostility) transforming the "forbidden" or "hidden meaning" into "the outer shell, the façade of the joke" (p. 55), that is, its "surface meaning" (Freud 1917/1961/1963, p. 103). Yet, Freud does not explicitly apply the terminology of "manifest-latent" to jokes, and in other works typically treats the "latent content" as unconscious, which it would not be in jokes, sarcasm, puns, and other figurative modes of expression. Thus, a crisp formalization of the manifest versus latent content distinction is desirable for a scientific treatment of multilayered linguistic materials (see also, Erdelyi 1985; 1999):

Let $\varepsilon$ be an event (an utterance, a dream, a mistake), and let $[\varepsilon]$ be some context, that is, a set of other events, external or internal (other utterances, free associations, attendant circumstances, known facts) which the subject brings to bear on the target event, $\varepsilon$. The manifest-latent content distinction arises from the conditionalization of the event, $\varepsilon$, on the context, $[\varepsilon]$, and is defined by the inequality,

$$
\varepsilon \neq \varepsilon \mid[\varepsilon]
$$

Thus, an isolated event may not mean the same thing as the self-same event when it is evaluated in a given context. The latent content, $\varepsilon \mid[\varepsilon]$, may be conscious (as in jokes or sarcasm) or unconscious (as in some dreams and errors).

Unduly reductionistic psychology has perennially focused on the surface, manifest content of psychological events, $\varepsilon$, while ignoring the underlying, latent meaning of the psychological event, $\varepsilon \mid[\varepsilon]$, which arises from the interaction of the event with its context. Radical behaviorism, in effect, ensconced a superficial conception of the stimulus in psychology, which was permitted to deal only with palpable ("observable") manifest phenomena. The cognitive revolution may not have fully moved past this phase in regard to repression, restricting its study largely to surface events, when the real action often unfolds at the deeper layer of latent contents, conscious or unconscious.

Thus, denial is not a perceptual failure in the sense that the perceiver fails to "see" $\varepsilon$, or even $[\varepsilon]$, but is a failure of insight, a failure to see - or to remember - the latent content, $\varepsilon \mid[\varepsilon]$.

\subsection{Elaborative or additive processes}

Denial richly partakes of both inhibitory and elaborative aspects of repression. It is inhibitory repression in the sense that $\varepsilon \mid[\varepsilon]$ is inhibited, but it is elaborative or constructive in that the person must decide on what constitutes the operative context, $[\varepsilon]$. (The context includes not only external events but also internal knowledge bearing on the target event.) A person "in denial" will not argue the facts (the $\varepsilon$ 's) but will argue about what constitutes its relevant context, $[\varepsilon]_{\mathrm{a}}$ versus $[\varepsilon]_{\mathrm{b}}$, and thus fails to extract the latent content or extracts a different latent content.

For research purposes, the issue may be framed as a question of weights assigned to the elements of the context, $[\varepsilon]: w_{1} \varepsilon_{1}, w_{2} \varepsilon_{2}, w_{3} \varepsilon_{3}, \ldots w_{i} \varepsilon_{i}, \ldots, w_{n} \varepsilon_{n}$. In effect, different weightings, including zero weightings, of each of the contextual elements, yields a different context, and therefore a different latent content. Determining the weights assigned by different subjects, implicitly or explicitly, would be an easy laboratory project, as would be the change over time of these weights (with the concomitant changes in the operative context and latent meanings).

If the weights can be thought of as values on an inhibition-excitation continuum, the underweighting of unwelcome contextual elements may be understood as another implementation of inhibition. It is quite clear from clinical or everyday examples of denial - see the Case of $\mathrm{N}$., in the next paragraph - that contextual elements are being weighted differently and tendentiously, with drastic changes in the meaning of target events. Thus, not only is $\varepsilon \neq \varepsilon \mid[\varepsilon]$, but $\varepsilon\left|[\varepsilon]_{\mathrm{a}} \neq \varepsilon\right|[\varepsilon]_{\mathrm{b}}$. Different operative contexts, which the person selects from both the external world and from internal knowledge structures, impart different meanings to particular target events. Thus, even without the massive repression of some event, biased forgetting and remembering of contextual elements can sculpt drastically different versions of the reality accessible to consciousness.

Another clinical fragment, The Case of N., is now considered, which illustrates concretely some of the points just discussed and makes a full transition to elaborative types of distortions that are the hallmark of a large swath of defensive processes.

This patient continually rails against homosexuals, whom he detests with a violent passion. After a brief, unsuccessful marriage, followed by impotence, he began to experience delusions of persecution, according to which the CIA and the FBI were continually observing him with the ultimate purpose of getting him to submit to the sexual advances of Richard Nixon. He gave up all attempts at heterosexual sex, because he would "not make love in public," that is, in front of the lurking agents. He soon came to understand also that his impotence had been imposed on him, via laser rays, by Nixon's agents. Satellites specifically sent up for the purpose began to bombard him with homosexual messages. Finally, he constructed a special protective metal hat, fitted with a complex electrical jamming device. He wore this hat continually, at home and in public places, including restaurants and work (he was soon dismissed). Even so, the messages that he should submit to Nixon increased in intensity and began to "penetrate" at times. Around this period he took all his jackets to a tailor and had the tailor sew up the slits (or flaps) in the back of the jackets. He implored his male acquaintances to do likewise, lest they be taken for "slot-jacket ass panderers." 
He deplored tight dungarees because they revealed buttocks too openly and therefore constituted a disgraceful invitation to sodomy. He complained that the CIA was spreading rumors that he was a homosexual; indeed they had contrived to find "doubles" for himself and a friend and photographed them - the doubles - in "disgusting" homosexual acts, all for the purpose of blackmail, so that he might submit to the homosexual importunings of the "anarcho-communist sodomite" Gerald Ford, the new President, who, as he now came to realize, was really "behind" the conspiracy. (Nixon, it turned out, was just a "front"). N. consciously hated homosexuals; indeed, the extent of his hatred was worrisome, for he began to mutter darkly about destroying them, seeing himself an innocent heterosexual victim of homosexual persecution. (Adapted from Erdelyi \& Goldberg 1979, p. 372, with permission)

$\mathrm{N}$. is in laborious denial of what seems to be a transparent homosexual disposition. At the manifest level, he presents himself as a heterosexual who hates homosexuals, but at the latent level, he is homosexually charged. Clearly, most of us would weight contextual elements differently than $\mathrm{N}$.

There are numerous elaborative transformations that are presumably intended to reduce N.'s consciousness for his underlying homoeroticism. Two notable examples are projection (in which the subject and object of a proposition are transposed, e.g., from "I love him" to "He loves me") and reaction formation (in which the predicate is reversed into its opposite, e.g., from "I love you" to "I hate you") (Erdelyi 1985; Freud 1909/1955). N. uses another elaborative distortion, rationalization (also emphasized by Bartlett), to explain his impotence: He cannot make love to women while being spied upon by the FBI and the CIA agents, and, moreover, Nixon's satellite-launched beams impede his functioning. $\mathrm{He}$ does not consider - or, gives zero weight to - the alternate notion that a homosexually inclined man might not be sexually aroused by women. He explains the "homosexual messages" he experiences as emanating from Nixon's satellites - in short, it is not he but Nixon who is the homosexual. His lurid homosexual fantasies are displaced upon the CIA-concocted "doubles." There are "rumors" about his being a homosexual but these are attributed to Ford. Apparently, zero weight is given to other reasons for such rumors. Symbolization is used copiously by $\mathrm{N}$. He constructs a mechanical defense mechanism, an impressive electronic helmet (he had been an engineer before his breakdown). This defense mechanism, however, fails him, for the homosexual messages get more intense and penetrate at times. He sews up the flaps on the back of his jackets, as if to say, "No entry!"

These strange elaborations or additions are presumably deployed by $\mathrm{N}$. to diminish consciousness for his own homoerotic cravings. He is too disturbed to manage the camouflage effectively, and so his efforts are sadly transparent to most observers. His psychological defenses, like his electronic helmet, are not working properly.

Is $\mathrm{N}$. ever conscious of his homoeroticism? Currently, we have no effective methodology for settling such a question in a given case. If we asked N., he would violently deny it, but it is likely that, as with other appetitive drives, his urges wax and wane, as might his degree of awareness for his cravings. Thus, in the case of unbearable drives, the degradation of the target material (by simple inhibition or more complex reconstructions) is not likely to lead to long-lasting amnesia.

\section{What happens to repressed (inhibited, suppressed) memories?}

If we instruct a subject to "reject and keep something out of consciousness," can the subject do this, and if yes, what happens to the "something"? Do we have laboratory data on this question? Of course, and in large quantities.

Dan Wegner (1989) has, for example, shown in programmatic research that instructing subjects not to think of a WHITE BEAR succeeds in keeping the thought out of consciousness (though not perfectly, since the idea does seep into ongoing thought to some extent). What happens if now the subject is freed from the task of repression (Wegner calls it "suppression")? Wegner claims, based on some of his data, that a rebound effect results - a return of the repressed/suppressed phenomenon - in which the subject becomes exaggeratedly involved - "obsessed" - with white bears. The experimental literature, actually, is mixed on this rebound effect, and some recent laboratory work has failed to obtain it (e.g., Anderson \& Green 2001; Gildston 1998). It is likely that different stimuli and different subjects will produce different outcomes. Also, different tactics for "keeping something out of consciousness" will likely be more or less effective. Not-thinking about some material may be made easier by else-thinking, that is, thinking of something else (e.g., the Rat Man's repression-by-prayer in sect. 2). As shown experimentally (e.g., Smith et al. 2003), exposure to massive interference can produce massive disruptions ("memory blocking") of target items, which, however, remain available, as shown by memory cuing.

There has accumulated, further, a considerable experimental literature on "intentional" or "directed" forgetting. Though there have been different explanatory stances taken toward the phenomenon, including selective rehearsal, selective search, and retrieval inhibition (I suspect all of them are partly right and that the effect is multiply determined), there is little question that forgetting can be intentionally induced (e.g., Epstein 1972; Geiselman et al. 1983; Roediger \& Crowder 1972; Weiner 1968). Ironically, this may be one of the most classic yields of laboratory psychology. In 1885, the founder of the experimental study of memory, Hermann Ebbinghaus, addressed the question of the fate of perfectly mastered memory materials (lists of nonsense syllables, in this case) when this material was "left to itself," that is, not attended to, thought of, and so on - an easy task with nonsense syllables (Ebbinghaus 1885/1964). The well-known Ebbinghaus "curve of forgetting" (never actually plotted by Ebbinghaus) shows that retention of material intentionally excluded from consciousness declines over time (and, apparently, without a rebound effect). Note that the forgetting is the result of the thought-avoidance, since diminished accessibility is evident when the repression is discontinued at testing. This inaccessibility, or forgetting, does not necessarily mean permanent loss or unavailability of the forgotten. Indeed, the obvious results of Ebbinghaus (as he himself viewed them) may be conceptually refractory, even misleading (Erdelyi 1996). Consider the following two questions: (1) What if memory were tested 
differently - or, to put it in starker theoretical terms what if there were no one memory but a multiplicity of memory systems, then would the single Ebbinghaus function describe the temporal dynamics of all memory systems? (2) What if repression (suppression, thought avoidance) were lifted and the opposite cognitive process instituted, namely, conscious effort to retrieve the previously avoided "something"? With this "work of remembering" (Breuer \& Freud 1895/1955; Freud 1914a/ 1958), can the forgotten be recovered? Or, put differently, can the Ebbinghaus curve reverse course and actually rise with time?

Much research has accumulated on both of these questions, and it has become clear that inaccessibility need not be general, nor permanent (e.g., Ballard 1913; Erdelyi 1996; Payne 1987; Roediger \& Thorpe 1978; Tulving 1983; 1987; Tulving \& Pearlstone 1966; Tulving \& Schacter 1990). It has become commonplace in modern cognitive psychology and neuroscience to posit the existence of multiple memory systems (e.g., "explicit," "implicit," "procedural") which produce contradictory or "dissociated" outputs. Clinical neurology had long since pointed to such memory dissociations, as in the classic report of Claparède (1911) of an amnesic patient unable to recognize Claparède from a previous encounter and yet able in some sense to remember Claparède, or at least his nasty behavior (shaking her hand while holding a pin between his fingers). As a former neuroscientist and the author of an 1891 book on aphasia, Freud recognized kindred phenomena in the psychological clinic, and he advanced the view that hysteric symptoms were "reminiscences" of materials inaccessible to consciousness (Breuer \& Freud 1895/1955). In modern times, huge neurological literatures have accumulated establishing the reality of neurologically based memory dissociations (e.g., Cohen \& Squire 1980; Corkin 1968; Parkin 1987; Tulving 1983). Further, the modern neuroscience literature has demonstrated that some amnesic patients show part-availability for what appears inaccessible: Despite their failure to remember events consciously, they nevertheless evidence unconscious priming effects (e.g., Weiskrantz et al. 1974) or produce errors that are semantically meaningful (Werner 1956).

Vast laboratory literatures have also accumulated on normal subjects that show similar unconscious priming effects with subliminal presentations: The subjects respond differently or with different reaction times to subsequent supraliminal test stimuli (e.g., Bargh \& Chartrand 1999; Bornstein \& Pittman 1992; Fisher 1956; Jacoby \& Witherspoon 1982; Marcel 1983a; 1983b; Pötzl 1917). In their influential paper, Jacoby and Witherspoon refer to such unconscious memory effects as "remembering without awareness."

Thus, different measures, or perhaps different memory systems, yield different levels of memory at different times, and so the Ebbinghaus function, though reliable, is circumscribed in its generality. The multisystem view implies an alternative to Freud's conversion hypothesis (which holds that the materials that are repressed from consciousness are converted into alternative modes of remembering, such as dreams, slips, symptoms, etc.). A simpler hypothesis (Erdelyi 1990; 1993; van der Kolk 1994 ) is that the alternative modes of remembering are not conversions of the repressed memories but spared memories. By this view, repression knocks out declarative (conscious) memories, but other memory systems (e.g., procedural ones, as in symptoms) are not similarly affected and continue to reflect remembering. (For relevant brain research on the dissociability of declarative memories from their emotional counterparts, see Bechara et al. 1995.) Freud may still turn out to be right, and psychological research may yet show that, once the repressed memory is recovered into the conscious channel, the memory stops expressing itself through the less direct channels. This is a researchable question.

The experimental literature does corroborate Freud's claim (Breuer \& Freud 1895/1955) that with "concentration" and repeated recall effort, the inaccessible may eventually be recovered into consciousness. In general, multiple testing produces memory increases (hypermnesia) over time. For example, in two studies reported by Erdelyi and Kleinbard (1978), subjects' recall of a list of pictures was repeatedly tested for a period of a week. Memory improved by more than $50 \%$ after the week of protracted testing. (To control for shifts in response bias, subjects were required to produce the same number of recalls in each trial, guessing if necessary.) The ErdelyiKleinbard retention curves look like upside-down versions of the Ebbinghaus function, with retention increasing rather than decreasing over time. Interestingly, the corresponding multi-trial tests of memory for words failed to produce memory improvement.

There are two discernible reasons for the difference between the Ebbinghaus and the Erdelyi-Kleinbard functions. The first, and most interesting for the repression issue, is that Ebbinghaus intentionally avoided thinking of the to-be-remembered materials (nondefensively repressed them), whereas the subjects in the Erdelyi and Kleinbard study did the opposite, namely, concentrated on the target material and tried repeatedly to retrieve the material. The second relevant factor is the type of stimulus involved: nonsense syllables in Ebbinghaus's case and pictures in Erdelyi and Kleinbard's successful tests. An overview of the laboratory literature by Payne (1987) suggests that there is a stark stimulus effect operating in the laboratory studies: Virtually all $(96 \%)$ of the studies using pictorial stimuli produced hypermnesia, whereas fewer than half $(46 \%)$ produced it with wordlists learned in the laboratory. The percentage of successful studies with nonsense syllables, moreover, is about $0 \%$ (Erdelyi 1996). This simple stimulus factor caused laboratory psychology, which was fixated on verbal and mostly meaningless materials, to doubt the existence of upward trending memory for half a century (see Erdelyi 1996). Obviously, the clinical literature invariably deals with highly meaningful materials and diverges in this respect from the artificial stimuli so standard in the older laboratory literature.

Actually, the recovery of inaccessible memories has been part of scientific psychology for more than a century (see Erdelyi 1996). The phenomenon was probed by P. B. Ballard in his 1913 monograph, "Oblivescence and Reminiscence." In most of his studies, Ballard used two recall trials, separated usually by one or two days' retention interval. In this two-trial situation, the following outcomes can (and do) occur. Some stimulus items are never recalled, that is, they are recalled neither in the first nor in the second trial. Another possible outcome is 
that some items are recalled on the first trial but forgotten on the second. Ballard designated such forgetting as oblivescence. The opposite outcome, or reminiscence, is observed when initially unrecalled items are recovered in the second trial. Finally, initially recalled items may be recalled again, that is, an item may be recalled on the first trial and then on the second test as well.

Ballard had the foresight to carry out his work with different types of stimuli, including poetry and nonsense syllables. Regardless of the stimulus used, Ballard found that both oblivescence and reminiscence occurred from first to second trial, but that the balance between the two varied with the stimulus. With poetry, for example, reminiscence exceeded oblivescence, resulting in an overall "improvement" of recall (which is today referred to as hypermnesia); with nonsense syllables, on the other hand, oblivescence exceeded reminiscence, for an overall decrement of memory (amnesia). Thus, Ballard was able to obtain Ebbinghausian results - amnesic memory when he used Ebbinghausian stimuli (nonsense syllables). When the stimuli become less artificial (e.g., poetry), the opposite, hypermnesic memory, was observed. Oblivescence may be ubiquitous, but so is reminiscence, and in this we see the paradoxical side of memory: Over time, memory simultaneously becomes better and worse, hence Ballard's title, "Oblivescence and Reminiscence." Thus, regardless of the stimulus used, there is always an up-trend and a down-trend in memory over time, a most convenient feature for a memory system involved in selectively enhancing or degrading memory.

Despite the beautiful simplicity of Ballard's discovery - that both reminiscence and oblivescence are fundamental to memory over time, and that their balance depends in part on the type of stimulus used - another grand-Bartlett effect imposed itself, this time in experimental psychology (see Erdelyi 1996). The memory literature following Ballard almost immediately began to confuse reminiscence with improvement (hypermnesia) and, attempting to replicate Ballard's improvement effect - typically with nonsense syllables - often failed in the effort. Researchers began to worry about the reliability of memory improvement, even though they were actually replicating Ballard's failure to get improvement with nonsense syllables. With the use of ineffective stimuli and the confusion of reminiscence with improvement, the field drifted into disarray. Claude Buxton, in his influential review of 1943, concluded that "reminiscence" was "a now-you-see-it-now-you-don't" phenomenon. Buxton, however, was no longer talking about reminiscence but of Ballard's "improvement" (what I call hypermnesia). In fact, reminiscence is a rock-solid phenomenon, on par with forgetting, and it is improvement, as Ballard had clearly shown, that may be chancy when the wrong stimulus is used.

Recent laboratory work (Erdelyi 1996, Ch. 8) has documented hypermnesic recall beyond a few days' retention interval, including an unexpected jump in recall level after periods of months. The multi-trial recall performance over some nine months of testing of a $9 \frac{1}{2}$-year-old child, Karina, for Bartlett's story, "The War of the Ghosts" (332 words long), was probed. Repeated recall effort in the first three months (atypically) failed to produce hypermnesia but after a six-months' fallow interval (in which there was no testing), a dramatic increase in recall level (by more than 200\%) was observed. False recalls, however, increased even more after the six-months interval. A Criterion-Controlled Free Recall (CCFR) measure for narrative recall (Erdelyi 1996, appendix), in which hits (true recalls) are conditionalized on a pre-set false-recall level, still showed a substantial jump in correct recall (of about $75 \%$ ) after the six-months interval. Thus, with false-recall level controlled, a big jump in recall level was still observed. Later research on Karina's $6 \frac{1}{2}-$ year-old sister, Maya, essentially replicated Karina’s long-term delayed recovery of memory, both with raw and controlled recall measures.

If we can trust such long-term but small-sample findings (though other laboratories have begun reporting hypermnesia effects over weeks, e.g., Bahrick \& Hall 1991; 1993; Herrmann et al. 1987), it would seem that the recovery of memories is a standard feature of repeated recall over time, both in short-term single-laboratory probes as well as over periods of weeks and months. It is true that false recalls are also churned up over time, but one phenomenon does not invalidate the other. Both occur. Roediger et al. (1997) focus on this "paradox" of memory, emphasizing that repeated recall enhances "accurate retention while sometimes leading to increased recall of wrong information" (p. 119). Signal detection theory (e.g., Macmillan \& Creelman 2004; Swets 1964) resolves this surface paradox by parlaying the functional relation between hit rates and false alarm rates into a single isosensitive function, the ROC curve. In the clinic, it is difficult, maybe impossible, to distinguish true recalls from false recalls because we don't have the original event against which to check the recalls. The laboratory, however, helps us cinch the phenomenon of recovery of (true) memories and of overall memory improvement, hypermnesia, because of the availability of the original stimulus.

Just as the laboratory has methodological advantages, so too it has its shortcomings. Especially from the standpoint of defensive repression, stimulus insufficiency is a problem, for we can hardly duplicate the kinds of "intolerable" or "unbearable" (Freud 1894/1962; 1896a/1962) stimuli that are the purview of real-life and the clinic. Moreover, it is clear from the clinical literature that what is "intolerable" varies vastly from person to person (just consider the two case histories discussed in Section 2: In the Case of B., the death of a brother; in the Case of N., homosexual cravings). Another refractory problem is the question of stimulus source. In the laboratory, the experimenter can control how many times and when the subject is exposed to the stimulus. In real life, the stressful events may be intermittently reproduced or cued. The Ebbinghaus curve would look different if intermittent re-exposures to the stimulus list occurred. Even more problematic is the reality that many of the "stimuli" that are repressed come from within and cannot be neatly manipulated, let alone controlled by an experimenter. In most clinical examples of defensive repression, including those of Freud, the crucial stimuli are biological irruptions of unbearable drives - possibly cued by environmental events - that require, as in the Myth of Sisyphus, a repeated renewal of effort. Thus, a substantial swath of the laboratory literature fails to sample the real-life situation and so suffers from questionable content validity.

With practice, the repressive process, like typing or driving, might be automatized and may not be particularly 
Erdelyi: The unified theory of repression

salient to consciousness (hence the long-standing assumption that repression is unconscious). When an increase in task difficulty occurs, as in the case of some significant new threat, conscious control may be partly reinstituted (as when a sudden problem arises in a driving situation). The task, also, may prove too difficult and repression may fail or partially fail. For example, repression may break down intermittently and yield oscillations of consciousness for the stressful events (DSM-IV; Horowitz 1986). "The return of the repressed" may also be observed for systemic reasons, as when the subject is disinhibited because of depleted "cognitive resources" due to other life stressors like illness, intoxication, or old age, or because of psychological state (e.g., sleep).

\section{Distortion by reconstructions and false additions}

On the strength of informal laboratory work, Bartlett (1932) forced experimental psychology to accept the reconstructive (and therefore distorting) character of memory. According to Bartlett, mental "schemas," reflecting cultural habits, biases, and logical expectations, intrude upon and distort memory. One of the "distortions" Bartlett emphasized was "omission." The clinical and laboratory data show, as we have seen, that at least this Bartlettian distortion can be partially reversed (in this respect, falsifying Bartlett's radical revisionist hypothesis that no memory trace for the original events exists beyond the current overwritten material). The work of remembering diminishes "omissions" but, as just noted, falls far short of eliminating them. Yet, just as nature abhors a vacuum, so psyche abhors gaps and actively tends to fill-in these gaps in a "fundamental effort after meaning" (Bartlett 1932, p. 227). In trying to recall degraded versions of the past, the subject not only recovers some of this material, but also schematically augments the degraded accessible memories for meaning. Schematic augmentation fills in the blind-spots of memory - just as it does our blindspots in perception. With both recovery and schematic augmentation unfolding over time, it is not surprising that both true recalls and false recalls are observed.

Now, here is the intersection between Freud and Bartlett: The constructions and reconstructions of Freud and Bartlett are the same but for motive. The concept of "schema," popular as it is, is fuzzy. Bartlett emphasized intellectual considerations, cultural habits, logical expectations, and so on. Such intellective schemas do not, however, preclude perfectly obvious emotional schemas (wishes, emotional attitudes, defensive needs). It is no accident that the terms are often overlapping, if not identical (see Table 1). Even on the point of motive the differences are ones of emphasis, due no doubt to differences in the stimuli. Bartlett, actually, points occasionally in the direction of defensive distortion, as when he states, "if the affect is displeasing, distortions are more likely to occur" (Bartlett 1932, p. 91). It strikes me as obvious that the different emphases of Bartlett and Freud of the kinds of schemas that structure distortions - intellective versus emotional schemas - arise from availability heuristic biases. The stimuli encountered in the clinic are substantially more emotional than those dealt with in the laboratory.
Table 1. Some overlaps between Bartlettian and Freudian distortions $^{a}$

\begin{tabular}{|c|c|}
\hline Bartlett (1932) & Freud (various) \\
\hline omission & omission, censorship, ellipsis \\
\hline rationalization & rationalization \\
\hline elaboration & secondary elaboration \\
\hline change in emphasis & displacement of accent \\
\hline $\begin{array}{l}\text { condensation, } \\
\text { importations, } \\
\text { transference }\end{array}$ & $\begin{array}{l}\text { condensation, displacement, } \\
\text { smuggled-in items }\end{array}$ \\
\hline items are symbolized & symbolization \\
\hline constructions & constructions \\
\hline interpretation & interpretation \\
\hline bias in recall & tendentious recall \\
\hline $\begin{array}{l}\text { imposed links of } \\
\text { connection, striving } \\
\text { after associative links }\end{array}$ & $\begin{array}{l}\text { false connections, } \\
\text { compulsion to associate }\end{array}$ \\
\hline distortion & distortion, falsification \\
\hline transformations & transformations \\
\hline $\begin{array}{l}\text { reversals (“opinions and } \\
\text { conclusions are } \\
\text { reversed” p. 128) }\end{array}$ & reversal, reaction-formation \\
\hline imaging & $\begin{array}{l}\text { imaging, plastic word } \\
\text { representation }\end{array}$ \\
\hline $\begin{array}{l}\text { curious perseveration } \\
\text { of the trivial (p. 184) }\end{array}$ & representation by triviality \\
\hline
\end{tabular}

${ }^{\mathrm{a}}$ Actual terms are set in boldface.

Although distortion and bias in information-processing are standard fare in cognitive psychology (Erdelyi 1974; 1985), motive is a difficult problem for science (e.g., Wegner 2002). Still, contemporary social-personality psychology is rife with motivated biases, including egotistical biases (e.g., Greenwald 1980; Paulhus \& John 1998), moralistic biases (Paulhus \& John 1998), trivialization (e.g., Simon et al. 1995), temporal bracketing, minimization (Baumeister et al. 1998), illusion of unique invulnerability (Perloff \& Fetzer 1986), illusion of control, positive illusions (Taylor \& Brown 1988; Taylor 1989), and the like. Worth noting also is a trend toward schema-like formulations of motivated biases. In a laboratory evaluation of the status of defense mechanisms, Baumeister et al. (1998) conceptualize defenses as efforts by individuals to sustain "preferred views of themselves" (p. 1082). In a similar vein, McAdams (1998) focuses on how we "make sense" (p. 1127) of our identities or life stories. Defense mechanisms are "viewed as narrative strategies that shape how a life is told" (p. 1127), and an "affinity between stories and human life" (p. 1125) is emphasized. It no longer seems such a chancy leap from "The War of the Ghosts" to the psychopathology of everyday life.

Martin Conway (1997b) presents a modern cognitive treatment of narrative constructions and reconstructions that are implemented by a "central cognitive processor" in which activation and inhibition occur at different substrates of autobiographical memory. As Conway notes, the narrative approach to autobiographical memory is not new. For example, the psychoanalyst Ernst Kris (1956/1975) advanced the concept of the personal myth, which 
individuals to a greater or lesser extent edit over time, omitting details that are emotionally inconsistent with the myth.

The one major defense mechanism which the present constructivist treatment does not obviously accommodate is regression, a defense involving a developmental retreat to more primitive styles of acting and thinking. Work in modern social-cognitive psychology provides a simple conduit to this defense in schema terms. The basic idea, though Bartlettian terminology is not typically used, is that the overall cognitive system is made up of multiple, often overlapping schemas (structures, systems, selves, states) that might differentially structure current inputs and that, depending on circumstances, different schemas might be deployed by the overall system (e.g., Horowitz 1988; Markus \& Nurius 1986).

Dan Gilbert (1991), in an analysis of cognitive belief systems, suggests multiple levels of truth-value processing, the more primitive system (the first phase of the "Spinozan" system) being a credulous one - as in Freud's "primary process thinking," it knows no no and believes whatever it apprehends or imagines. The more advanced informationprocessing system - as in Freud's “secondary process thinking" - is a critical system that performs "a secondary psychological act" (Gilbert 1991, p. 108): It consciously and effortfully evaluates the output of the earlier credulous system and either accepts or "unaccepts" the current belief. Gilbert shows that when the overall cognitive system is stressed or cognitively overloaded, there is a tendency for the more sophisticated system to drop out and the more primitive belief-system to prevail. In effect, Gilbert is proposing a regression from a more advanced to a more primitive mode of analysis as a result of cognitive stress or depletion. In this vein, Gilbert also emphasizes the paradox of facilitation through disinhibition, a leitmotif of psychoanalysis and neuroscience (e.g., Damasio 1994; Freud 1900/1953; 1917/1961/1963; 1920/1955; Gazzaniga 1985; LeDoux 1996; Pavlov 1927; Pötzl 1917). As in psychoanalysis, when the executive system (the ego) is inhibited or undermined (by overload, sleep, intoxication, therapy, brain damage, old age), the more primitive system becomes disinhibited, and the output of the overall system is infused with the content and style of the primitive system. As both Pavlov and Freud emphasized, disinhibition yields recovery of memory - "spontaneous recovery" of extinguished responses (Pavlov 1927) or "the return of the repressed" in dreams or indirect behavior channels such as symptoms (Freud 1900/1953; 1917/1961/1963; 1920/1955).

Similar themes can be discerned in terror management theory, which focuses specifically on defenses against the terror of death (Pyszczynski et al. 1999; 2000). Research suggests that when our mortality is in focal consciousness, we tend to use proximal defenses, which basically distort the facts in question. When mortality issues are primed but are subliminal or at the "fringe" of consciousness, distal defenses are deployed, which, in effect, operate on schemas themselves - for example, on one's "cultural world view," which becomes exaggerated and less nuanced.

The modern experimental literature on memory has extended traditional interference manipulations that had led to an emphasis of retroactive and proactive interference in forgetting (e.g., Jenkins \& Dallenbach 1924; McGeoch 1932; for overviews, see Baddeley 1976; Crowder 1976; Erdelyi 1996) and merged them with the Bartlettian constructivist theme that memories are malleable and subject to a multiplicity of distorting effects, including "memory illusions" or outright memory delusions (e.g., Ceci et al. 1996; Conway 1997a; Jacoby et al. 1989a; 1989b; Loftus 1979; Loftus \& Ketcham 1994; Neisser 1982; Roediger et al. 1997; Schacter 1999).

Thus, in highly influential research by Elizabeth Loftus and her colleagues (e.g., Loftus 1979; Loftus \& Palmer 1974; Loftus et al. 1978), memory distortions were experimentally induced through the use of suggestive querying ("post-event information"). When subjects were asked, for example, "Did another car pass the red Datsun while it stopped at the stop sign?" in a scene that actually had no stop sign, they were more likely to remember the phantom sign. Similarly, if a question were phrased alternatively, "About how fast were the cars going when they smashed/hit each other?" different speed estimates were generated as a function of the wording; also, subjects exposed to the "smashed" version of the query, were more likely to report seeing "broken glass," even though none had been present. Roediger and his colleagues (e.g., Roediger \& McDermott 1995; Roediger et al. 1997) have similarly demonstrated that confabulatory memories can be created through associative implication. Employing a paradigm introduced by James Deese (1959), they have shown that a false memory could be induced by presenting items that semantically implied a common target that was never actually presented. For example, a list containing the items bed, rest, awake, tired, dream, wake, and snooze tends to yield the false remembering of the semantically implied (but never presented) item, sleep. As already noted, Roediger and his colleagues have also emphasized that the very act of repeated retrieval often produces the effect of increasing both true memories as well as false memories. Also, repeatedly thinking about a "non-event" (i.e., engaging in fantasy) often yields false remembering of the non-event (Ceci et al. 1996). These experimental demonstrations provide laboratory analogs of self-induced memory distortions, since, presumably, whatever the experimenter can induce in the subject, the subject can also induce in the subject.

What has been tellingly omitted from most of this literature is the extent to which Freud's conception of mental processes, including memory, both anticipated and extended this narrower constructivist tradition. The elemental point of Freud's psychology is that what is accessible to consciousness is never the true, unfettered information, but highly worked-over reconstructions (brought about, for example, by the "dream work," "defense," the "joke work," and similar transformational techniques). Thus: "Re-activated memories ... never re-emerge into consciousness unchanged" (Freud 1896a/1962, p. 170); recall is an "amalgam" of fact and fiction; we "construct memories almost like works of fiction" and therefore "there is no general guarantee of the data produced by our memory" (Freud 1899/1962, p. 315); what returns in the "return of the repressed" are often "false recollections" (e.g., Freud 1901/1960, p. 6) or "substitute formations" (e.g., Freud 1915a/1957/ 1963, p. 111); and so on.

For this reason, though it seems not to be appreciated in the modern literature, the recovered memory movement that has so energized the scientific community (e.g., Banks \& Pezdek 1994-1995; Kihlstrom 1997; Loftus \& Ketcham 1994) is antithetical to Freud's psychological 
stance that the "historical truth" (e.g., Freud 1937/1964) of recovered memories cannot (nor should) be ascertained by the therapist. The originators and perpetuators of the memory recovery movement (e.g., Bass \& Davis 1994) were not psychoanalysts - often not psychologists. Their program, which fundamentally violated psychoanalytic technique, would have been rejected by Freud, who, early on, underscored the "growing recognition of the untrustworthiness of statements made by witnesses," even when testifying about themselves (Freud 1906b/ 1959 , pp. 103-113). Although Freud had originally proposed his "infantile seduction" theory of hysteria from the problematical recollections of some of his early patients, for which he has been much criticized (e.g., Crews 1995), Freud retracted his erroneous proposal within a decade (e.g., Freud 1906a/1953). Thereafter, Freud emphasized the role of fantasy in such recollections: "If hysterical patients trace back their symptoms to traumas that are fictitious, then the new fact that emerges is precisely that they create such scenes in phantasy" (Freud 1914b/1958, p. 17). Such recollections tend to be, even if they are not invariably, "imaginary memories" (Freud 1906a/1953, p. 274). Ironically, if Freudian tenets had not been so muffled in modern psychology, much of the miscarriage of concept and practice might have been averted and the corrective programs by experimental psychologists, such as Beth Loftus, rendered less necessary.

\section{Conclusion}

Psychology has become pervasively constructivist (as neuroscience and psychoanalysis have always been). It is by now the standard view that memory is not strictly veridical and is subject to wide-ranging and ongoing distortions. The source of these distortions - omissions, elaborations - varies from situation to situation, and one type of distortion need not be incompatible with another: Sometimes the distortions come from intrusions from other lists; other times, from implicit suggestions in the querying process; other times, from long-established internal structures, which sometimes are intellectual in character (Bartlett) and other times emotional (Freud). All these distortions can be exacerbated by the subject himself who, in the process of thinking about and retrieving information, may inhibit memories or amplify errors of previous constructions in a process akin to succumbing to one's own propaganda and the creation of myth.

As scientists, we should resist such trends in our own work and should not ignore the historical facts. Progress by euphemistic relabelling or tendentious silences, though ubiquitous in the real world, is not scientific progress. It does not matter from a scientific standpoint if we labelled the process "repression," "suppression," "retrieval inhibition," "dissociation," "cognitive avoidance" - or "Mary" - though such untrammelled synonymy, without the anchor of viable theory, drifts, over time, into dissociated baronies of discourse and brings upon us a selfinflicted curse of Babel: We do not understand each other; we misunderstand each other; we impose distinctions where none are justified; we fail to make distinctions that are obligatory; we argue about the wrong issues and fail to deal with the real issues; we dissociate ideas that belong together; we suffer amnesia and consign ourselves endlessly to rediscover what we already have mastered.
The laboratory and the clinic have converged on a simple but fundamental insight: Cognition, from perception to memory, is pervasively constructive. We structure our fragmentary reality by omitting from and elaborating on our meager scraps of information. We inhibit and augment our reality by different techniques and for different reasons. We try to make sense of our reality, intellectually as well as emotionally.

\section{ACKNOWLEDGMENTS}

For helpful comments on earlier drafts of this article, I thank George Bonanno, Martin Conway, Richard Lazarus, Beth Loftus, Neil Macmillan, Laraine McDonough, Arthur Reber, Mark Solms, and Diane Zizak. Many thanks are due also to Michael Anderson and Stephen Ceci for their extensive feedback in their capacity as reviewers of earlier drafts of the submitted manuscript. Finally, my special thanks to Robert Viscusi, poet and magician of prose, for occasional guidance in the underworld of irony from ancient times to the present.

This work was supported by a Fellowship Leave Award from Brooklyn College and by PSC-CUNY grants from the Research Foundation of the City University of New York.

\section{NOTES}

1. Anna Freud might have agreed. In a telling conversation with Joseph Sandler (Sandler \& A. Freud 1985), published posthumously, she anticipates one of Daniel Holender's (1986) criticisms of experimental psychology's efforts to demonstrate nonconscious priming: The prime might have been fleetingly conscious but forgotten by the time the experimenter tests for it. Here is how Anna Freud treats the conundrum in an interchange on the defense mechanism of reaction formation:

Anna Freud: Heinz Hartmann would say that it can become automatic.

Joseph Sandler:... I still think that there must be an awareness of the impulse to evoke the response.

Anna Freud: Hartmann and I discussed it at the time, in 1936 and 1937. There must be a momentary awareness. (Sandler \& Freud 1985, pp. 22-23)

Charles Eriksen, the great critic of subliminal perception, later proposed also the idea of "automatization" of highly practiced behaviors (Eriksen \& Pierce 1968), now a common notion in cognitive psychology.

2. A foreshadowing of the present twofold organization of repression can be found in Freud's The Interpretation of Dreams (1900/1953): "There can be no doubt that the censoring agency, whose influence we have so far recognized in limitations and omissions in the dream-content, is also responsible for interpolations and additions to it" (Freud 1900/1953, p. 489).

\section{Open Peer Commentary}

\section{Encouraging the nascent cognitive neuroscience of repression}

Michael C. Anderson and Benjamin J. Levy

Department of Psychology, University of Oregon, Eugene, OR 97403.

mcanders@uoregon.edu http://memorycontrol.uoregon.edu

blevy@uoregon.edu http://memorycontrol.uoregon.edu 
Abstract: Repression has remained controversial for nearly a century on account of the lack of well-controlled evidence validating it. Here we argue that the conceptual and methodological tools now exist for a rigorous scientific examination of repression, and that a nascent cognitive neuroscience of repression is emerging. We review progress in this area and highlight important questions for this field to address.

To mainstream experimental psychologists, repression has always had the scent of an untestable theory, too exotic to be true, based too much on clinical anecdote. Given the importance of this construct in the realm of clinical observation for more than a century, however, one is compelled to ask why more empirical progress has not been made. In his target article, Erdelyi hints at two conceptual errors that have marginalized repression and limited scientific progress. First, the characterization of repression as a specialized psychological defense mechanism hindered investigators from distinguishing between the cognitive mechanisms that might underlie repression (e.g., inhibition) and the use to which those mechanisms are put (e.g., defense). Second, the historically enforced distinction between suppression and repression insists that repression is unconscious, rendering it difficult to study scientifically. Erdelyi's target article persuasively argues, however, that Freud never intended repression to be exclusively unconscious, and that it is entirely reasonable to study mechanisms separate from the reasons for engaging them. If so, the necessary tools to understand repression are readily available in the armamentarium of cognitive psychology and cognitive neuroscience. The conclusion couldn't be more different from what mainstream experimentalists have supposed: Repression is, in fact, a scientifically tractable problem.

We strongly agree (Anderson 2006; Anderson \& Green 2001; Anderson \& Levy 2002). We disagree, however, with Erdelyi's conclusion that if suppression = repression, then much of the controversy surrounding repression "dissolves." This seems premature. One can accept that suppression (repression) exists without believing that this process could be responsible for cases of memory recovery of the sort addressed in the recovered memory debate, around which much controversy revolves. Although the extension of work on inhibition to recovered memories is plausible, it is not yet proven. In fact, we would like to suggest that the scientific study of repression is in a place not unlike where false memory research was in the early 1990s (e.g., Loftus 1993; Loftus \& Ketcham 1994).

Loftus and colleagues had successfully demonstrated that misleading post-event suggestions can alter what subjects believe they remember (e.g., Loftus et al. 1978). Whereas these demonstrations generated useful evidence regarding the fallibility of eyewitnesses, they concerned fairly trivial details of an experience (e.g., whether a car had passed a stop sign or a yield sign) that should never be regarded as evidence for "memory implantation." Indeed, early claims that misinformation effects could potentially generate entirely false memories of traumatic experiences were appropriately criticized (e.g., Harvey \& Herman 1994; Olio 1994; Pezdek et al. 1997). However, programmatic research on false memory has gradually extended suggestibility findings in ways that render this claim more plausible (Loftus \& Pickrell 1995; Mazzoni \& Loftus 1998; Mazzoni et al. 2001). Many investigators now consider it possible that some instances of memory recovery result from suggestion. These important results were not accomplished without patiently building an evidence base with progressively greater ecological validity.

As in the early 1990s, cognitive psychology now has several model paradigms through which to study memory control, including the think/no-think paradigm (Anderson \& Green 2001), directed forgetting (e.g., Bjork \& Bjork 2003), and retrieval-induced forgetting (Anderson 2001; Anderson et al. 1994; Bjork et al. 1998; for reviews, see Anderson 2003; Levy \& Anderson 2002). Consider the think/no-think paradigm. Subjects view reminders to previously encoded memories and, while focusing on each reminder, do one of two tasks: either retrieve the memory associated to the reminder (respond trials), or exclude the memory from consciousness (suppression trials). As Figure 1 illustrates, whereas retrieving the associated memory improves later retention, excluding traces from consciousness impairs memory relative to baseline items. Thus, when people are inclined to be reminded, reminders enhance memory as nearly everyone might guess; but when people desire not to be reminded, the reminders not only fail to enhance memory, they set the occasion for processes that impair memory. This reversal of the normal positive influence of reminders is a product of executive control mechanisms mediated by

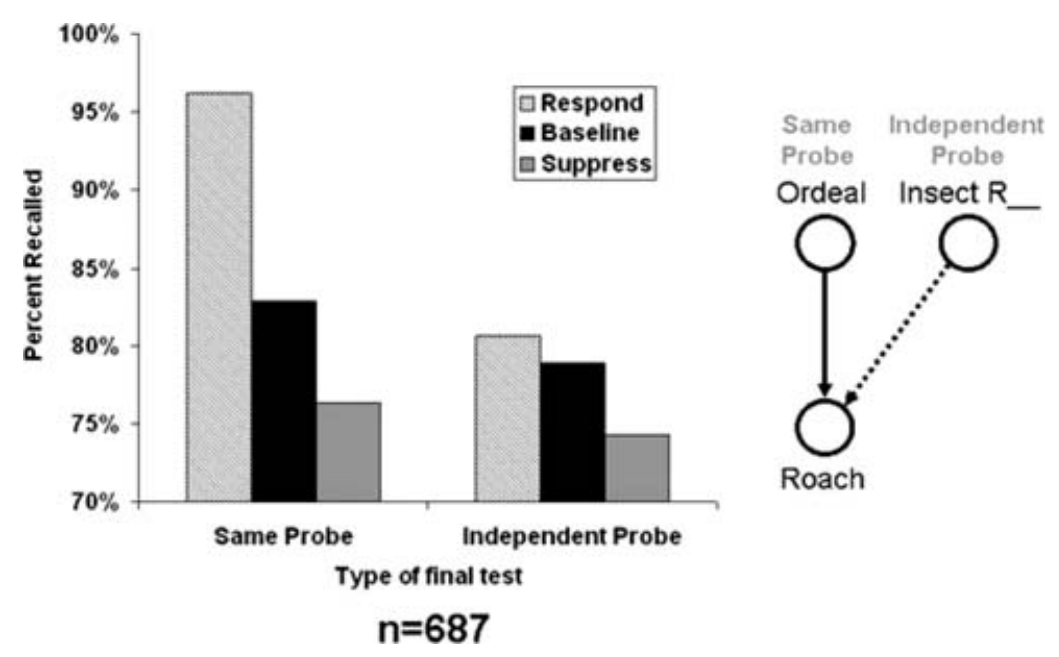

Figure 1 (Anderson \& Levy). Final recall in the TNT procedure from a meta-analysis of 687 subjects. The graph shows recall on the final test as a function of whether subjects recalled the item (Respond), suppressed the item (Suppress), or had no reminders to the item (Baseline) during the think/no-think phase. The left side shows recall to the originally trained cue (i.e., the Same Probe); the right side shows recall to a novel, extra-list category cue (i.e., the Independent Probe). The large difference between the respond and suppression conditions reflects the total memory control effect, of which positive control (facilitation of respond items above baseline due to subjectinitiated retrieval) and negative control (inhibition of suppression items below baseline due to subject terminated retrieval) are components. Note that even if below-baseline inhibition did not occur, tendentiously avoiding retrieval when reminders are present clearly deprives a memory of reactivations (due to intentional retrieval or to spontaneous reminding) that otherwise would preserve and enhance more desirable traces, as Erdelyi discusses. 
dorsolateral prefrontal cortex modulating activation in the hippocampus (Anderson et al. 2004). Anderson and colleagues have argued that these findings provide an existence proof of mechanisms that could underlie repression (Anderson 2006; Anderson \& Green 2001; Anderson et al. 2004). Freud (1915a/1963) defined repression as "simply the function of rejecting and keeping something out of consciousness" (p. 147), which is precisely what we asked our subjects to do. Thus, it simply is no longer reasonable to say that there is no way that repression could occur.

However, to conclude, based on these findings, that inhibitory control underlies traumatic memory repression, though plausible to some (certainly to us and perhaps Erdelyi), is as warranted as concluding that false memories of abuse can be implanted based on misremembering a yield sign. Several issues remain to be addressed to build more ecologically valid support for this hypothesis. First, can inhibition suppress complex multi-modal memories for emotionally arousing events thought to be central to repression? Second, can inhibition endure for an extended time, or does it need to be continually reinstated? Third, what triggers recovery, and what are a memory's characteristics, once recovered? Fourth, might suppressed memories exert unconscious influence on behavior, through priming or conditioning? Finally, might inhibition progress from being intentional to being unintentional? The development of habitual diversionary thoughts through many experiences with avoiding a memory may render exclusion so routine that the original purpose of the diversions may be forgotten. Might the resulting retrieval-induced forgetting recurrently reinstate inhibition (Anderson 2001; Anderson \& Green 2001) without people's awareness?

Fortunately, significant progress has already been made. For instance, memory inhibition in the think/no-think paradigm can be obtained with emotionally negative stimuli (Anderson \& Kuhl 2004; Joorman et al. 2005), even when those stimuli are aversive photographs (e.g., car accidents; Depue et al. 2006). Interestingly, both retrieval-induced forgetting (Barnier et al. 2004; Wessel \& Hauer 2006) and directed forgetting have now been observed with autobiographical memories (Barnier et al., in press), even when the memories are recorded over multiple weeks in a diary and contain emotionally significant events (Joslyn \& Oakes 2005). Others have demonstrated that inhibition in the think/no-think paradigm affects explicit, but not implicit, memory (Kawaguchi et al. 2006), showing persisting influence of inhibited information outside of awareness (see also, Bjork \& Bjork 2003). Although some reports indicate that retrievalinduced forgetting dissipates after 24 hours (MacLeod \& Macrae 2001; Saunders \& MacLeod 2002), others have now observed these effects, undiminished, after a week (Storm et al., in press; see also Migueles \& Garcia-Bajos, submitted). Importantly, we have found that people with more extensive history of trauma (of any sort) show enhanced memory inhibition (Anderson \& Kuhl 2004), establishing a clear connection between these processes and those likely to be used to control unwanted remindings in daily life. These findings validate the point, stressed by Erdelyi, that individual differences will turn out to be crucial. All that it takes for inhibition to be a reasonable model of repression is for there to be some individuals who can wield it effectively. Indeed, although the overall size of the inhibition effect in the think/no-think procedure is modest $(7-10 \%)$, some individuals show effects as large as $60 \%$, even though the total time spent suppressing is only a little over one minute. Why? Understanding this variation is a vital goal in the coming years.

Although the science of repression is in its early stages, the burgeoning knowledge about the cognitive and neural basis of executive control, long-term memory, attention, and affect regulation will surely provide a powerful theoretical basis through which to understand how the human mind exerts control over unwanted memories or feelings. At this early stage, what is required most of all to transform the nascent cognitive neuroscience of repression is patience, a long-view of progress, and a skeptical stance regarding the overly damning and tendentious critiques of the most strident skeptics of repression. With any luck, Erdelyi's target article will entice a generation of talented researchers to understand how humans adapt memory in the aftermath of trauma, and experimental psychology will no longer sweep repression under the rug.

\section{Can repression become a conscious process?}

\section{Simon Boag}

Department of Psychology, Macquarie University, Sydney, NSW, 2109, Australia.

simon.boag@psy.mq.edu.au http://www.psy.mq.edu.au/staff/sboag/

Abstract: A major weakness in Erdelyi's account concerns the claim that repression can become conscious. A relational account of cognition demonstrates that if repression is successful, then the repressive act cannot become known. Additionally, "resistance" further distinguishes "repression" from "suppression." Rather than blurring the distinction between these processes, it is possible to recognise a series of defences. Suggestions are provided for alternative research avenues.

Erdelyi's target article provides an important contribution to understanding repression within modern psychological thinking. However, a major theoretical weakness in his account, involving the claim of a "false" distinction between repression and suppression, concerns whether repression can become conscious. Such an assertion necessitates a discussion of what is meant by "conscious" and "unconscious," but Erdelyi's reference to an "unconscious-conscious" continuum (comparing the issue to the arbitrary "child-adult" distinction), is uninformative since it is without reference to "knowing" (an issue similarly neglected by others within this debate - e.g., Cramer 2000). Subsequently, the conceptual coherency of Erdelyi's position remains unclear. A helpful direction to address this is a relational account of consciousness, where cognition (understood here in terms of acts of knowing, such as believing and remembering) is viewed as a relation between a cognising subject (a knower) and an independent object term (a situation, or state of affairs) that is known (Anderson 1927/1962; Maze 1983; Michell 1988). ${ }^{1}$ To be "known" is a relation entered into, rather than a quality of property of situations known, so for a mental act $p$ (where $p$ may be a desire or belief that $p$ ), to be conscious is simply for $p$ to be currently known (such that subject $S$ currently knows $p$ ), and to be unconscious means simply that $p$ is not currently known. The act of knowing is, itself, not automatically known but requires attending to for it to become conscious. That is, when $S$ knows (or wishes, etc.) of some situation $p$, the relation of knowing (or wishing, etc.) (call this relation $S R_{p}$ ) is itself unconscious and does not become conscious unless it becomes the object of a second mental act, such that $S$ knows $S R_{p}$. For example, at a specific time $S$ becomes aware of $p$, and then at a later time $S$ is prompted to pay attention to the fact of becoming aware of $p$ ( $S$ knows that $S$ knows $p$ ). This awareness of $p$ can now be called conscious, whereas previously it had been an unconscious mental act, or descriptively unconscious (Freud 1923/ 1961, p. 13). Furthermore, any process will remain unconscious if the causal antecedents necessary for its becoming conscious fail to occur. Thus Freud correctly recognised that "every psychical act begins as an unconscious one, and it may either remain so or go on developing into consciousness" (Freud 1912/1958, p. 264).

Repression, itself, can be conceptualised as the act of turning away from, and inhibiting, wishes and desires that are believed to engender threat (Freud 1915a/1957; 1926a/1959). On the foregoing analysis, for repression to become conscious would mean that it is capable of becoming the object of a second mental act. That is, when $S$ represses the wish for $p$ to be the case (again, a relation, $S R_{p}$ ), that repressive act can be known 
$\left(S\right.$ knows $S R_{p}$ ). However, a problem emerges here for Erdelyi's account, not because repression cannot ever be known by the repressing subject, but due to the consequences of repression, itself. As Freud notes, repression prevents more than just the target from becoming conscious, because not only is the "instinctual impulse ... in some way inhibited, its precipitating cause, with its attendant perceptions and ideas, is [also] forgotten" (Freud 1939/1964, p. 128). That is, if repression is successful, then the connection with the repressed material prevents the act itself from becoming conscious.

The reasoning for this is as follows: To know that same act of repression $\left(S R_{p}\right)$ requires knowing the terms involved in that act, which requires knowing $p$. However, if repression is successful, then it is no longer possible to know $p$, and so it would no longer be possible to know the repressive act, $S R_{p}$, until the repression is lifted (and even then it would not be automatically conscious but only so if becoming the object of a second mental act). Thus, even if the repressive act is known when repression first occurs, knowledge of this would not be possible after the act. Furthermore, the clinical phenomenon of "resistance," central to Freudian repression (cf. Madison 1961), and which Erdelyi fails to address, reveals that repression is more than simply ignorance that can easily be corrected (Freud 1910b/ 1957 , p. 225). Instead, resistance occurs despite $S$ 's "conscious" intention to know the repressed (Freud 1910a/1957, p. 30), indicating that the repressed is actively prevented from being known unconsciously (which raises certain theoretical questions concerning how this could occur - see Maze \& Henry 1996; Boag, in press). "Suppression," on the other hand, appears to involve a subject $S$ knowing the target $(p)$, suppressing knowledge of it (such that $p$ is no longer currently known), but both readily recalling $p$, and the act of suppression $\left(S R_{p}\right)$, itself (Anspaugh 1995; Juni 1997). Rather than blurring the distinction between repression and suppression, then, it may be more helpful to simply recognise the existence of a series of defensive operations, as Freud indicated:

Between repression and what may be termed the normal method of fending off what is distressing or unbearable, by means of recognising it, considering it, making a judgement upon it and taking appropriate action about it, there lie a whole series of more or less clearly pathological methods of behaviour on the part of the ego. (Freud 1936/1960, pp. $245-46)^{2}$

Erdelyi's general thesis is not incompatible with this position, although he appears to want to conflate repression with suppression to make the existence of repression indisputable. However, this is unnecessary because the existence of "inhibitory processes," of which repression is a species, is incontrovertible, and serves as an important avenue for empirical research of the motivated cognitive distortions that Erdelyi refers to. Freudian repression itself is best understood in terms of cognitive-behavioural inhibition (see Boag 2006), a conceptualisation which finds many parallels in contemporary psychology (e.g., Fox et al. 2005; Nigg 2000). Exploring the neural mechanisms of such processes provides a solid empirical platform for investigating psychodynamic processes, and of particular interest is the relationship among the prefrontal cortex, "behaviour inhibition," and the mediation of conflicting responses, as discussed within modern neuroscientific research (e.g., Cardinal et al. 2002; Ridderinkhof et al. 2004). Thus, while Erdelyi's proposal requires further conceptual development, his paper is an important contribution to bringing repression into the framework of contemporary empirical psychology.

\section{NOTES}

1. This viewpoint has a long history, spanning back to Aristotle (see Petocz 1999), through medieval scholasticism (see Pasnau 1997), and emerging in both the American new realists (e.g., E. B. Holt) and British realists (e.g., G. E. Moore, J. Laird, and Samuel Alexander; see Michell 1988). The position is also prominent in the Australian school of Andersonian Realism (see Anderson 1927/1962; 1930/1962; Baker 1986).
2. However, there is no clear difference in quality between unconscious and conscious processes, on the one hand, and normal and pathological behaviour, on the other (see Petocz 1999, p. 232).

\section{Motive and consequence in repression}

\section{Joseph M. Boden}

Christchurch Health and Development Study, Department of Psychological

Medicine, Christchurch School of Medicine and Health Sciences, Christchurch 8011, New Zealand.

joseph.boden@chmeds.ac.nz

Abstract: Erdelyi's unified theory of repression offers a significant advance in understanding the disparate findings related to repression. However, the theory de-emphasizes the role of motive in repression, and it is argued here that motive is critical to the understanding of repression as it occurs in the mental life of individuals.

Matthew Erdelyi's article, "The Unified Theory of Repression," presents a theoretical framework from which to view the vast array of findings on the phenomenon of repression. In this commentary I examine the implications of a unified view on our understanding of repression, particularly in terms of the role of repression in the ecology of the mental life of the individual.

Motive. One main feature of Erdelyi's unified theory is that it posits that the process of repression arises from the normal operation of attention and memory; it occurs as a function of human cognition due to the wide array of stimuli that may be impinging upon consciousness at any time. To support this argument, Erdelyi distinguishes between the process of repression itself, and the defensive purposes (the motive) repression frequently serves. The distinction between process and motive permits the use of evidence from a wide range of experimental literatures on attention and memory that had been previously thought to be unrelated to repression.

In separating process from defense, however, we may lose the concept of motive in our understanding of repression as it occurs in the ecology of mental life. While we have gained an understanding of how repression works, we are less equipped to understand why it occurs. It could be argued that motive is a critical aspect of repression; indeed, Freud referred to repression as inhibiting "unbearable" mental contents (Freud 1915a/1963), putting motive at the center of repression. A view of repression as the motivated forgetting or avoidance of unwanted mental contents gives us some purchase on the reasons why these processes may be employed, and allows us to link the behavior to informational threat in the environment.

Also, motive may also be a key in understanding why the use of repression becomes automatic (and therefore unconscious). Erdelyi shows that repression may be either conscious or unconscious, and there is extensive evidence for unconscious repression. As Baars (2002) points out, one of the apparent functions of consciousness is to facilitate learning, resulting in the automatizing of various processes. Once automatized, these processes are no longer under conscious control. If we assume that repression involves (in at least some instances) an automatic process, then the concept of motive allows us to link learning - a motivated acquisition of skills - with the automatizing of repression.

A further role for motive in the understanding of repression is a link to individual differences in the use of repression. A wellestablished line of research has examined individuals thought to use repression habitually; this individual difference is referred to as the repressive coping style (e.g., Weinberger 1990; Weinberger et al. 1979). Research on the repressive coping style has linked this to alterations in attention, memory for negative stimuli, autobiographical memory, and a range of other behaviours, generally elucidating the effects that repression may have on cognitive functioning (Derakshan \& Eysenck 1997; Weinberger 1990). 
Consequence. Erdelyi's unified theory offers a framework for understanding the myriad, and in many cases confusing and contradictory, findings regarding the consequences of repression. For example, although some studies have shown differences in memory for negative stimuli among repressors (e.g., Ashley \& Holtgraves 2003; Boden \& Baumeister 1997; Davis \& Schwartz 1987; Holtgraves \& Hall 1995), others have failed to demonstrate these differences (e.g., Boden \& Dale 2001; Brosschot et al. 1999; Schimmack \& Hartmann 1997). Although it is clear that the use of repressive defense has implications for later information processing, it is unclear what the specific consequences of repression are.

Erdelyi argues that it is possible that repression encompasses a wide variety of information processing biases, including attention biases, forgetting, elaboration, and other cognitive alterations. On this view, repression could be viewed as an informationprocessing bias, rather than a discrete cognitive function. It is quite clear that the use of a panoply of defenses would result in an increase in noise in experimental designs; for example, a study designed to assess the nature of memory deficits in repressors following an unpleasant stimulus might yield contradictory or null results, simply due to the fact that the repressors in the experiment are actually using different (defensive) means to accomplish the same ends.

If indeed repression is better described as a kind of bias across a series of cognitive functions, rather than a specific function itself, there are a number of implications for further research. First, it would be useful to study any covariation between the nature of threatening stimuli and the cognitive functions involved in repression; it may be that certain functions "work better" with certain kinds of stimuli. Second, it would also be useful to determine whether there are individual differences at work in the use of particular functions in the service of repression (e.g., Brosschot et al. 1999; Shane \& Peterson 2004b; Terry \& Burns 2001). Erdelyi and others (e.g., Boden \& Baumeister 1997) have argued that repression is not limited to repressors, but is probably a behavior that is common to all individuals; it is simply more easily measured under laboratory conditions in individuals who are thought to use it more habitually or with less provocation. It is unclear whether the kind of repression used to cope with, for example, thoughts of grief over the death of a loved one, is the same as the repression used to cope with everyday annoyances.

Summary. Erdelyi's unified theory of repression offers a significant advance in understanding the phenomenon of repression. It allows us to view repression as a bias in the operation of human cognitive functioning and, as such, allows us to better understand the disparate results of experimental studies of repression, and to proceed with further research that will aid in understanding the phenomenon. However, it is important to note that, while the process of repression and the (defensive) motive to repress must be disentangled for this advance to occur, the concept of motive is one that is critical to the understanding of the role of repression in the ecology of mental life.

\section{The illusion of repressed memory}

\section{George A. Bonanno}

Department of Counseling and Clinical Psychology, Teachers College, Columbia University, New York, NY 10027.

gab38@columbia.edu

Abstract: Erdelyi's unified theory includes the idea that traumatic memories can be unconsciously repressed so that they are enduringly inaccessible to deliberate recall. I argue here that clinical evidence for repressed memory is illusory, and illustrate this claim by examining previous studies of putative repressed memories and also recent research on nonverbal behaviors among survivors of childhood sexual abuse.

Erdelyi's attempt, in the target article, to integrate the variegated literature on motivated memory distortion and inhibition is impressive in its breadth and scholarly conviction. However, his fundamental thesis, that literally all manner of behaviors associated with the processing, distortion, and inhibition of traumatic memory are essentially the same, is both ineffective and hazardous - ineffective, because it blurs the rich interactions between motivation and mechanisms charted out over decades of experimental research; hazardous, because it seeks to lend credence to the fairy-tale belief that enduring repressed memories are possible. I will limit my remarks to the latter.

Let's not begin with Freud. Why quibble over what he did or didn't say? As Erdelyi acknowledges (a "grand-Bartlett effect"; sect. 2 , para. 5), both lay and professional theorists nearly uniformly associate the word repression with the idea that we can unconsciously bury traumatic memories so that they are unavailable to conscious recall for long periods of time. But, as myriad reviews have shown (e.g., Holmes 1973; McNally 2003), there is simply no solid evidence for this idea. What we can do - and here there is abundant evidence - is render traumatic memories relatively benign, not by unconsciously repressing them but through active inhibition of their emotional component (e.g., Foa et al. 1995b). When inhibition is effective, memories are still accessible, but they are less upsetting, and less gripping. Indeed, this is the core aim of exposure treatments (Foa \& Rothbaum 1998).

Conviction in the illusory reality of repressed memory is fueled, as it has been since Freud, by superficial clinical evidence. A recent compelling example being Williams' (1994) interviews of women with documented childhood sexual abuse (CSA), occurring approximately 15 years earlier. Remarkably, $38 \%$ seemed to have failed to recall the target CSA event. Had they repressed it? Closer inspection suggests more mundane factors (Loftus et al. 1994). For example, most (67\%) of those who did not report the target abuse did recall other abuse events; they simply failed to distinguish the target event from other repeated abuse experiences. Additionally, 9\% of Williams' sample was age 3 years or younger when the target abuse occurred, and thus would not have reliably encoded the event. Also, as Williams noted, an estimated $4 \%$ to $8 \%$ of CSA reports are fictitious (Everson \& Boat 1989). Finally, there is the possibility of deliberate nondisclosure. Unreported abuse was highest when the perpetrator was a family member. Because many CSA survivors remain in contact with family perpetrators, it is often easier to keep the past a private matter.

A study by Femina et al. (1990) even more poignantly illustrates the potential for misconstruing intentional nondisclosure as repression. These investigators interviewed another sample with documented abuse histories, and again a compelling subset $(26 \%)$ seemed to have failed to recall the index event. However, when Femina et al. invited the failed-recall participants for an additional "clarification" interview in which they first attempted to establish greater rapport and then confronted them with the discrepancies in their prior report - all participants acknowledged the index abuse. They each had a reasonable explanation for deliberately withholding the information. One participant hoped to avoid further familiar stress (e.g., "My father is doing well now. If I told now, I think he would kill himself"; Femina et al. 1990, p. 229); another wanted to keep the memory private because it was too upsetting, and she wanted to forget about it; still another stated she withheld disclosure simply because she didn't like the interviewer.

Erdelyi resurrects Freud's idea that repressed memories, buried in the unconscious, find expression in hidden (latent) meanings, as if memories themselves could function as autonomous, goal-directed beings; a conception he notes that "experimental psychology often treats ... as suspect" because of 
"disagreements about the interpreted latent contents" (sect. 3.2.2, para. 3). Rightly so; consider for example a recent study in which my colleagues and I (Bonanno et al. 2002) asked a sample of adolescent girls with documented CSA histories to describe in detail the most distressing events they had ever experienced. We made no mention in that interview of sexual abuse. However, we did follow with a second, more structured trauma interview in which for the first time we explicitly asked the girls about prior CSA. Consistent with other studies, one-third of the CSA survivors did not voluntarily disclose a past abuse experience. However, when we established a solid rapport and then later questioned them directly, all but two participants acknowledged the abuse.

What was particularly compelling was how much intentional nondisclosure seemed like repression. Nondisclosers exhibited greater nonverbal signs of shame. On the surface, this has the decidedly "psychoanalytic" feel of an unconscious expression of repressed abuse. Yet, shame has been shown to be an explicit gesture of appeasement - a nonverbal acknowledgment of a perceived moral transgression that the expresser is aware of, indeed painfully so, but would rather not talk about (Keltner \& Buswell 1997; Tangney 1999). If the memory were truly repressed, such feelings would have been kept at bay.

Nondisclosers also made more non-Duchenne smiles. Genuine (Duchenne) smiles are marked by contraction of the muscles around the eyes and associated with genuine positive emotion. Non-Duchenne smiles are not accompanied by an experience of positive emotion and do not involve contraction around the eyes. Rather non-Duchenne smiles are polite social signals and often mask other emotions (Duchenne de Bologne, 1862; Ekman \& Friesen 1982; Frank et al. 1993). But there is nothing unconscious about these expressions; whereas Duchenne smiles occur spontaneously, non-Duchenne smiles are best characterized as "deliberate attempts to influence the behavior or judgment of another person" (Bonanno et al. 2002, p. 97); in this case, to smooth over the intentional decision not to disclose a painful memory.

In the end, as Erdelyi notes, repression is just a word. But it is a word mired in imprecision, and unfortunately linked indelibly to the idea of the wholesale burial of traumatic memories. We can't forget the word, but we can frame it appropriately as a historical concept that should no longer hold spell over contemporary psychological theory.

\section{What Erdelyi has repressed}

\section{Frederick Crews \\ Department of English, University of California, Berkeley, CA 94720-1030. fredc@berkeley.edu}

Abstract: Erdelyi's "unified theory of repression" attempts to rehabilitate psychoanalytic doctrine by exaggerating its compatibility with the findings of cognitive science. In addition, Erdelyi treats Freud's writings as holy writ, any portion of which can be quoted to prove a point. He also relies on a long-discredited account of Freud's "seduction theory" and ignores important links between Freudian assumptions and our recent recovered memory movement.

Erdelyi's target article afforded him an opportunity to address the many grave objections that have dogged psychoanalytic knowledge claims, especially regarding memory. Instead, he has sought to define away the trouble. His "unified theory of repression" is not a theory but a transparent attempt to tuck one disputed tradition safely behind the skirts of a more scientifically respectable one. Ironically, his own concluding admonition constitutes the most appropriate verdict on his effort: "Progress by euphemistic relabelling or tendentious silences ... is not scientific progress" (sect. 6, para. 2).
Erdelyi's contention is that nothing substantive is at stake among quarreling memory theorists. They needn't worry, for example, whether repression - whose authenticity he treats as axiomatic - operates consciously or unconsciously, because support for each of those clashing views can be found somewhere in Freud's writings. Once Erdelyi has embraced this remarkably unscientific approach to repression and, further, has emptied the concept of its controversial psychodynamic content, repression supposedly proves to be the very thing that cognitive researchers have isolated in their laboratories.

Erdelyi cannot show a consistent belief on Freud's part that repression operates both consciously and unconsciously. Rather, he cites "ambiguous" and "self-contradictory" passages indicating that Freud was confused and careless, indeed downright incoherent, on this point. But Erdelyi, like a biblical fundamentalist, assumes that whatever the holy text declares at any juncture must be based in reality. If Freud, however opportunistically, asserted both $x$ and not-x, then the human mind itself must be so constituted as to enable both possibilities. QED.

Throughout Erdelyi's target article, we read of what Freud "discovered" and "realized" and "demonstrated" regarding memory. All such statements beg the question of how Freud corroborated his alleged findings. If he "demonstrated," for example, that dreams and jokes operate according to the same mechanisms, where did he take cognizance of other theories about those matters, and by what means did he show that his own explanations were more parsimonious and cogent than rival ones? How many subjects did he study experimentally, and what controls did he adopt against the risk of suggestion? Erdelyi contents himself with anecdotal single-case reports resembling Freud's own fast-and-loose, rhetorically coercive yarns.

Because Erdelyi reads Freud "biblically," he can overlook still another obstacle, namely, the difficulties the master encountered when applying his fatally vague concepts. Thus Erdelyi invests confidence in the theoretical apparatus of Breuer and Freud's Studies on Hysteria of 1895 without reflecting that, just a year later, Freud embraced a quite different conception of traumatic memory - a conception that he soon abandoned in favor of still another unsupported idea. Although Freud realized, serially, that his explanations had led to implausible corollaries and to therapeutic failure, he repeatedly clung to most features of the discredited theory and conjoined them with new, equally arbitrary, assertions about memory and trauma. At no point did he provide any empirical support for his shifting tenets. But Erdelyi endorses some of those tenets unquestioningly - being careful, however, to direct our gaze away from their idiosyncratic particulars.

Erdelyi takes pains to dissociate psychoanalytic doctrine from the modern recovered memory movement (even while quixotically citing some of its principal theorists as reliable experts on the nature of memory). Freud himself, according to Erdelyi, came to realize that the "problematical recollections of some of his early patients" (sect. 5, last para.) were merely fantasies. If only we had attended more carefully to Freud's warning against "the untrustworthiness of statements made by witnesses," Erdelyi writes, we could have spared ourselves our recent agony over false-memory accusations (sect. 5).

Erdelyi falls into several kinds of error here:

1. Modern recovered-memory cases have typically been generated not by "witnesses" whose recall may be faulty but by therapeutically induced convictions that such-and-such a misdeed must have been perpetrated long ago against the nonremembering patient, who then is coaxed into producing corresponding "memories" of the nonevent. This was precisely the reckless therapeutic style of Freud himself, both before and after he invented psychoanalysis.

2. Erdelyi overlooks important facts about Freud's "seduction" period that were established 20 years ago by a psychoanalytic investigator (Schimek 1987; see also Esterson 2001; Israëls \& Schatzman 1993). Freud lied or misremembered when claiming, 
much later, that his "seduction" patients' stories had been mere fantasies. His three relevant papers of 1896 show unmistakably that the "stories" were Freud"s own hypotheses, which he insistently tried to get his flabbergasted and protesting clients to corroborate. After that fiasco, he proceeded to build "psychoanalysis proper" on this very quicksand: the oedipal fantasies that he now purported to excavate were his own creation, largely recycled from his unsuccessful attempts to impose histories of early abuse on incredulous patients. From the outset, then, repression was nothing more than an excuse for misascribing Freud's own bizarre "stories" to other people's unconscious minds.

3. The psychoanalytic, as well as the prepsychoanalytic, Freud did lay claim to an ability to unearth veridical early memories through therapy. See, for example, the Wolf Man's alleged presence at a parental "primal scene" - an event that served a typically dramatic confirmatory function in Freud's case history, but that the patient himself later declared, convincingly, to have been Freud's implausible invention (Obholzer 1982).

4. Erdelyi overlooks a number of other traditional Freudian beliefs that were adapted by our recovered memory therapists: that repression explains why early experiences are inaccessible; that dredging up repressed traumas is the royal road to cure; that therapeutic suggestion can be safely discounted; that sexual experiences and thoughts are uniquely pathogenic; and that contemporary behaviors and dreams, once symbolically decoded, can reliably indicate the reality of otherwise unknown early events. Needless to say, all of this is sheer folklore, and very pernicious folklore, too.

The most rigorous studies of repression in its distinctively meaningful sense, as a mechanism that renders psychic contents inaccessible to consciousness, have found no believable evidence of its existence (e.g., Holmes 1990; Pope et al. 1999). Erdelyi's diluted and denatured version of the concept, however, would seem applicable to his own amnesia for obvious and often repeated objections to his views.

\section{Freud did not anticipate modern reconstructive memory processes}

\author{
Allen Esterson ${ }^{\mathrm{a}}$ and Stephen J. Ceci ${ }^{\mathrm{b}}$ \\ a $1 A$ Cromwell Grove, Hammersmith, London W6 7RQ, United Kingdom; \\ ${ }^{\mathrm{b}}$ Human Development, Cornell University, Ithaca, NY 14853. \\ allenesterson@ compuserve.com http://www.esterson.org/ \\ sjc9@cornell.edu
}

Abstract: In this commentary, we challenge the claim that Freud's thinking anticipated Bartlettian reconstructive theories of remembering. Erdelyi has ignored important divergences that demonstrate it is not the case that "The constructions and reconstructions of Freud and Bartlett are the same but for motive" (target article, sect. 5).

In his article, Erdelyi writes about what "Freud's clinical experience revealed" (target article, Abstract) with no indication that he is aware that Freud's reporting of his clinical experiences has been called into question (Esterson 1993, pp. 11-93; Webster 1995, pp. 136-167). A case in point is Erdelyi's alluding, in section 5, to patients' "recollections" of early childhood experiences that Freud later construed as "imaginary memories" (Freud 1906a/1953, p. 274). Modern scholarship has shown to be false the received story that in 1895-1897 most of Freud's patients told him they had been sexually abused in early childhood (Cioffi 1974/1998; Eissler 2001, pp. 94-117; Esterson 1993, pp. 11-31; 1998; 2001; 2006; Israëls \& Schatzman 1993; Scharnberg 1993, pp. 113-268; Schimek 1987). In this same sentence cited by Erdelyi, Freud writes that the repression of patients' childhood memories (of infantile masturbation) results in "phantasies (or imaginary memories)" being "produced during the years of puberty." So, on Freud's theory, (analytically inferred) unconscious "memories" of infantile masturbation metamorphose into (analytically reconstructed) "unconscious [sexual abuse] phantasies" at a specific age, a mysterious process that is scarcely consistent with Bartlettian reconstructive theories.

Erdelyi supports his claim of the Bartlettian nature of Freud's conceiving that "what is accessible to memory is never the true unfettered information" by quoting (in sect. 5, para. 10) as follows: "Re-activated memories ... never re-emerge into consciousness unchanged" (Freud 1896a/1962, p. 170). The remainder of Freud's sentence reads: "what becomes conscious as obsessional ideas and affects, and take the place of the pathogenic memories so far as conscious life is concerned, are structures in the nature of a compromise between the repressed ideas and the repressing ones." So what Freud is discussing are not Bartlettian distortions of memory, but the wholesale conversion of a supposedly repressed memory of an infantile sexual experience into something very different - obsessive actions and ideas that bear no obvious relationship to the experience. Two pages later Freud provides a clinical example: "A psychical analysis" (of obsessive behaviour) "shows that ... they can always be fully explained by being traced back to the obsessional memories which they are fighting against" (Freud 1896a/1962, p. 172). In a footnote Freud then outlines an analysis of the bedtime rituals of an obsessional patient, tendentiously interpreting each element in the rituals to be consistent with the patient's having been sexually abused in infancy by a servant girl, in line with his preconceived theory of obsessional neurosis at that time (see Esterson 1998, pp. 4-6; 2001, pp. 332-33; 2006). So, on Freud's theory, the "re-activated memories" that Erdelyi cites are manifested in the form of obsessive rituals, a process that can scarcely be said to illustrate Bartlettian reconstructive processes.

In the same paragraph (sect. 5, para. 10), Erldelyi alludes to Freud's notion of "substitute formations" (Freud 1915a/1957, p. 154) as illustrative of his Bartlettian credentials. The passage in question relates to the supposed repression of an idea that represents an instinct, with the resultant production of affect, such as anxiety (Freud 1915a/1957, p. 153). The example Freud provides immediately afterwards (p. 155), taken from the Wolf Man case history, involves an infant boy's "libidinal attitude towards the father" being "subjected to repression," resulting, by some mysterious process of "displacement along a chain of connections which is determined in a particular way," into phobic anxiety about wolves: "The result is fear of a wolf, instead of a demand for love from the father" (p. 155). The motive force for the repression was fear of castration, which Freud describes as "one of the commonest and strongest motives for repression" (1933/1960, p. 87). It is difficult to see much relation between this process and Bartlettian theories of memory. (See Esterson 2003, pp. 158-159.)

Another of Erdelyi's citations (sect. 5) purportedly demonstrating Bartlettian processes (Freud 1899/1962, p. 155) relates to Freud's theory of "retrogressive screen memories," which involves the repression of a contemporary idea and its projection back onto an early childhood memory trace to produce an enhanced memory (Esterson 2003, p. 157). However, the "screen memories" process as described by Freud depends heavily on the acceptance of his analytic interpretative procedure. An early memory of the yellow color of flowers has been enhanced by the repression of later sexual fantasies: "Taking flowers away from a girl means to deflower her" (1899/1962, p. 316); a memory of a childhood tooth extraction has been enhanced by the repression of memories of masturbation (1900/1953, pp. 390-391). Moreover, the memory is "formed" at a specific time (1899/1962, p. 322), in contrast with Bartlettian schematic modification of memory, in which each retelling further alters the features of the encoded event (Bruck et al. 2002).

The remaining citation from Freud's writings in the paragraph in question (sect. 5, para. 10), that pertaining to "false 
recollections" (Freud 1901/1960, p. 6), relates specifically to his theory of the forgetting of proper names. The quoted words concern a rather far-fetched analysis he has just given of the forgetting of a person's name and its (tentative) replacement by a different one. However, the "mechanism of false recollection" as described here requires a process of repression, not one of modification over time. Moreover, Timpanaro has examined this very example that Freud uses to exemplify his theory of the forgetting of names, and shown that it can be explained perfectly adequately without appealing to Freudian repression processes (Timpanaro 1976, pp. 63-65, 70-71, 75-77, 85-89).

Although Erdelyi is correct in noting the similarity between reconstructions based on emotional motives and those based on cognitive schemes, neither of these incremental reconstructive biases resemble the production of erroneous memories as described in the passages he cites. Of course it is possible to highlight certain elements in Freud's writings about memory that overlap with Bartlettian theories (e.g., his saying that "recall is an amalgam of fact and fiction"), but if the full context of Freud's accounts are taken into consideration, it is apparent that every one of the truncated quotations that Erdelyi cites in the paragraph in question relates to theories of memory that bear little relationship to Bartlettian reconstructive processes over time.

\section{The social psychology of cognitive repression}

\author{
Jennifer J. Freyd \\ Department of Psychology, 1227 University of Oregon, Eugene, OR \\ 97403-1227.
}

jjf@dynamic.uoregon.edu http://dynamic.uoregon.edu/

\begin{abstract}
Erdelyi identifies cognitive and emotional motives for repression, but largely neglects social motivations. Yet social pressure to not know, and implicit needs to isolate awareness in order to protect relationships, are common motives. Social motives may even trump emotional motives; the most painful events are sometimes the most difficult to repress. Cognitive repression may be impacted by social information sharing.
\end{abstract}

That people sometimes distort, omit, deny - repress ${ }^{1}$ - information is indeed obvious, as articulated in Erdelyi's insightful and timely target article. But why do individuals repress information? Erdelyi explicitly refers to only two categories of motive: cognitive and emotional. Erdelyi has neglected a third important category of motive for repression: social. Social motives include all the reasons individuals repress as a consequence of social pressures to not know and to doubt memories, and in response to an implicit need to isolate information from awareness in order to maintain a whole host of social (intimate, communal, political) relationships.

Campbell (2003) notes that "political struggles over memory are extraordinarily intense" (p. 52). Whether a struggle over memory for the events witnessed at Mai Lai (Freyd 2001) or the more mundane experience of discussing experiences of the day, social response to testimony will shape what is remembered (Fivush et al. 1997). Sometimes forgetting and unawareness are explicitly encouraged by others - for instance, when harmful events are perpetrated by one person or group of people on another with manipulations to reduce the subsequent disclosure and memory of those events (Veldhuis \& Freyd 1997).

Individuals are sometimes exposed to information that may endanger their well-being. In such cases, repression may be adaptive. Childhood abuse perpetrated by a caregiver is an example. Betrayal trauma theory (BTT; Freyd 1994; 1996; 2001; see Fig. 1) proposes that the way in which events are processed and remembered will be related to the degree to which a negative event represents a betrayal by a trusted, needed other (Sivers et al. 2002). Full awareness of such abuse may only increase the victim's risk by motivating withdrawal or confrontation with the perpetrator, thus risking a relationship vital to the victim's survival.

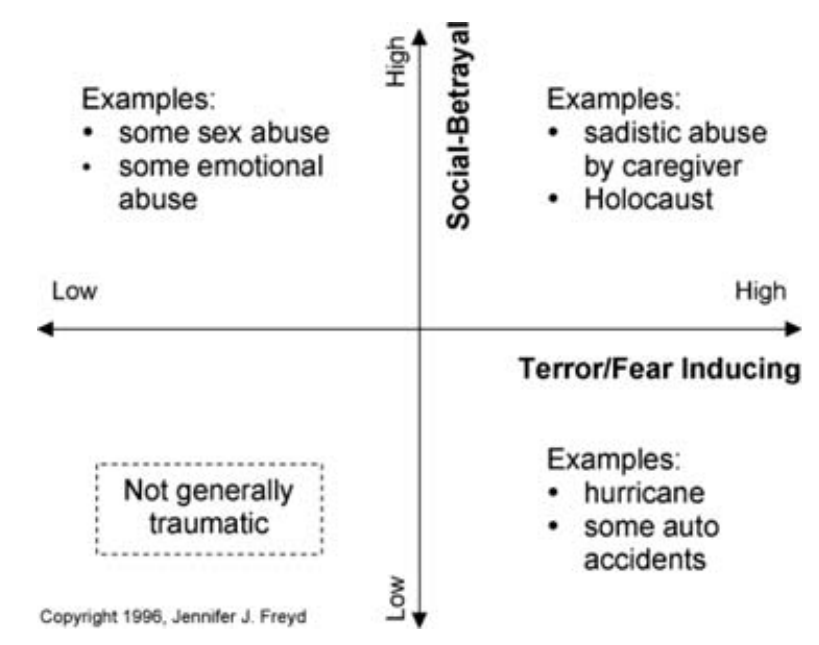

Figure 1 (Freyd). Two-Dimensional Model for Traumatic Events. Social betrayal is hypothesized to be associated with greater unawareness and reduced recall.

BTT posits that knowledge isolation is predicted by the threat the information poses to the individual's attachment system. Studies indicate reduced recall in the case of abuse by caregivers or close others (e.g., Edwards et al. 2001; Freyd et al. 2001; Schultz et al. 2003; Stoler 2001; Sheiman 1999; Williams 1995; for additional analyses see Freyd 1996). Freyd et al. (2001) found that physical and sexual abuse perpetrated by a caregiver was related to higher levels of self-reported less persistent memories of abuse compared to non-caregiver abuse (see Fig. 2).

Exposure to high-betrayal traumas is also associated with higher levels of dissociative tendencies (Chu \& Dill 1990; DePrince 2005; Plattner et al. 2003). Dissociation, like repression, may help keep betrayal information out of awareness. DePrince and Freyd (2004) found that under divided-attention, high dissociators have impaired memory for words associated with trauma (e.g., incest) but not for neutral words, as compared with low dissociators. Similarly, Becker-Blease et al. (2004) found that, under divided attention, abused children remembered fewer abuse-charged pictures relative to non-abused children.

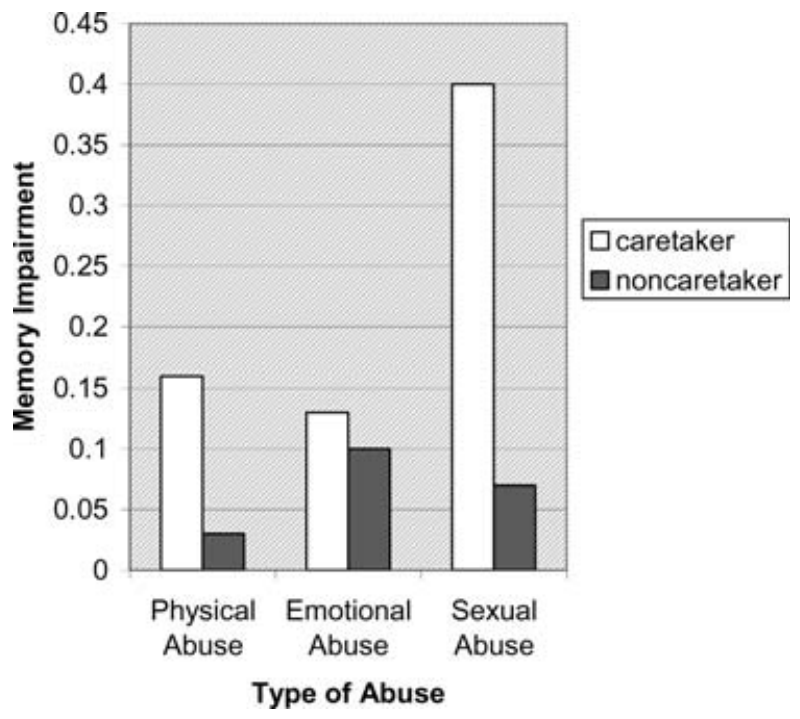

Figure 2 (Freyd). Average memory impairment (reduced recall range is 0 for excellent memory to 1.0 for very poor memory for abuse) for caretaker (high betrayal) and non-caretaker (lower betrayal) sexual, physical, and emotional abuse. (Results from Freyd et al. 2001.) 
What about betrayals, such as adultery, that do not meet criteria for abuse - are these also repressed? One striking phenomenon is when adultery is obvious to those outside the relationship, but the wronged partner is apparently unaware of the betrayal. I have conducted interviews with individuals who later discovered they had been in exactly this situation. In each case my informants described feeling dependent upon their partners. This appears to be betrayal blindness (Freyd 1996; 1999) - by not knowing about the adultery, a crisis is forestalled and a necessary relationship is "protected."

Awareness of betrayal is destabilizing. Zurbriggen (2005) has argued that a similar process can occur when people systematically remain unaware of observable betrayals by their own government. One motivation for remaining unaware is likely dependence upon the government for protection from threat. Shay (1994) also pointed to betrayal (particularly by commanding officers) as a key factor in soldiers' harm from war. War memories are among those must susceptible to forgetting (Elliott 1997; Grinker \& Spiegel 1945; Sargant \& Slater 1941; Thom \& Fenton 1920).

It is commonly stated that the primary motive for repression is avoidance of psychic pain. Yet pain is needed to motivate behavior, and lack of pain can be dangerous. Rather than avoidance of pain per se, I argue that repression is most likely when it serves a function such as protecting a necessary relationship. When betrayal is fully perceived, the resulting pain may motivate withdrawal or confrontation. In a betrayal trauma, this reaction could result in an even worse situation for the betrayed person; thus repression is adaptive. There is an analogous effect for physical pain - if mobility is sufficiently urgent for survival, even a serious injury may be ignored. The usual processes that produce severe pain and related unwillingness to move can be spontaneously suppressed in the right circumstances. This is not due to a desire to avoid pain, but a survival need that overrides the usual pain/immobility response. So, too, for psychic repression. Consistent with this, it is apparently more difficult to forget the death of one's own child than many other traumatic experiences (Elliott 1997).

Sharing or not sharing information about events has implications for the memorability of those events. A lack of social sharing of information may lead to decreased opportunities to rehearse information, encode it in more shareable and stable forms (Freyd 1983; 1990; 1996), and eventually impact recall (Freyd 1996; 2003). Consistent with this, child sexual abuse has a strikingly low disclosure rate and also has some of the highest rates of reduced recall (Elliott 1997; Freyd et al. 2005). Similarly, Foynes et al. (2006) found that non-disclosure of childhood abuse was predicted by the closeness of the perpetrator, just as reduced recall is so predicted (Freyd et al. 2001).

Although Erdelyi makes great progress toward a unified theory of repression, he largely neglects a crucial aspect of repression - the social psychological forces and motives. It is of course the case that individual nervous systems instantiate psychological repression, but a full understanding of the phenomena will not occur if the individual psyche is studied in a vacuum. Betrayal and other social forces must be part of a unified theory of repression.

\section{NOTE}

1. The words "repress" and "repression" are contested and often avoided, but in this context I follow Erdelyi's target article, which is defining and reclaiming these words.

\section{Forging a link between cognitive and emotional repression}

\author{
Esther Fujiwara $^{\mathrm{a}}$ and Marcel Kinsbourne ${ }^{\mathrm{b}}$

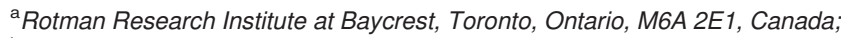 \\ ${ }^{\mathrm{b}}$ Department of Psychology, New School University, New York, NY 10003. \\ efujiwara@rotman-baycrest.on.ca kinsboum@newschool.edu
}

Abstract: Erdelyi distinguishes between cognitive and emotional forms of repression, but argues that they use the same general mechanism. His discussion of experimental memory findings, on the one hand, and clinical examples, on the other, does indeed indicate considerable overlap. As an in-between level of evidence, research findings on emotion in neuroscience, as well as experimental and social/personality psychology, further support his argument.

Erdelyi's unifying theory convincingly addresses the extensively used, yet frequently misunderstood, concept of repression. He shows the similarities between early definitions in psychodynamic and experimental psychology, literatures largely oblivious of each other. His key point is that repression should be understood as a mechanism regardless of its function, rather than as inherently defensive. Repression becomes a universal gatekeeper of the contents of consciousness, irrespective of underlying psychodynamic assumptions and of the nature of to-be-repressed information. Examples, mostly from the experimental memory literature, illustrate the similarity of distortions that render information inaccessible, whether for cognitive or emotional reasons. The question remains: If repression as a mechanism comprises suppression, inhibition, avoidance, and the like, why retain such an embattled concept? The justification reverts to psychodynamic traditions and therefore to defensive or emotional forms of repression.

Defensive repression is primarily a phenomenon of emotion, not memory. Repression of memory content is just one of many possible consequences. Under some circumstances, emotional information may be "repressed" on account of an anticipated or associated unwanted emotional state, rather than because the memory content per se is unwanted (or instructed not to be wanted). Erdelyi summons substantial support from the memory literature for the view that the means by which these forms of repression function are essentially the same. We propose to show that, in fact, the link between the experimental and the clinical findings is even closer than appears from the evidence presented in the target article.

Erdelyi cautions against inducing unbearable stress in the laboratory so as to mimic trauma and potentially trigger defensive repression, thereby questioning the validity of inferences from experimental to clinical findings. However, endocrinological and social psychology research that utilized various forms of stress induction found memory performance that is well in line with Erdelyi's assumptions. For example, experimental manipulations of stress-hormone levels show evidence for both memory enhancing and memory-impairing effects. Consistent with Erdelyi's comment about temporal shifts from hypermnesia to amnesia, delicate temporal dynamics of hormonal shifts seem to be a mediating variable. Administering adrenal glucocorticoid before memory retrieval generally impairs performance, whereas before memory encoding, it enhances performance (Het et al. 2005). This dissociation also obtains with psychosocial rather than direct hormonal stress manipulation (Kuhlmann et al. 2005).

Further, to forge the link between cognitive and emotional forms of repression, the extensive body of literature on the so-called repressive coping style is worth considering in more detail. Erdelyi questions the manner in which repressive coping style is usually established (by questionnaire measures: individuals with low self-reported anxiety and high social desirability). He prefers that individuals be selected by their ability to repress (not-think of) some target material, regardless of the reason. What would probably be achieved by such approach is the classification of individuals by their abilities in working memory, executive control functions, fluid intelligence, and so forth. This would provide a basis for personality differences in repressive abilities if, but only if, repression is studied as a general mechanism. However, for the emotional aspect of repression, the investigation of individual differences in defensive repression forms a critical link between experimental and clinical psychology, and this should not be underestimated. Is conventionally measured repressive coping style indeed associated with a higher capability for general (and not just defensive) repression, and is the mechanism the same in 
both cases? Research with individual differences in defensive repression allows more direct testing of the suggested similarity of basic experimental and clinical phenomena: Does naturally occurring repression (i.e., repressive coping style) correspond to (non-defensive) repressive abilities in the laboratory? Given a high natural motivation to repress negative affect, does implicitly probing for defensive repression parallel mechanisms of instructed, non-defensive repression? What situational variables are likely to trigger defensive repression? The latter question has been extensively studied in social psychology. The evidence supports processes of self-denial rather than just social impression management, which can be seen as a parallel to psychodynamic defenses (e.g., Myers \& Derakshan 2004). Moreover, in order to avoid the - by definition - biased self-reports of individuals with a repressive coping style, psychophysiological measures can be, and have been, used instead of questionnaires to identify individuals with higher or lower awareness of their own emotional state (e.g., Bonanno et al. 1995). Hence, such an approach parallels the assumption that repression is consciousness lowering, here operationalized as habitually lowered awareness for one's own emotions.

It may be more effective to use personality variables for approximating naturally occurring repression in the laboratory, than to equate the intensity of potentially to-be-repressed, traumatic laboratory stimuli. Further, assuming that unconsciousness is not a necessary condition for repression to occur, convergence between cognitive and emotional repression may be found in studies of emotion regulation. For example, Ochsner et al. (2004) reported that down-regulation of one's own emotions (i.e., emotion repression in a wider sense) may evoke brain activity that is very similar to that found in unemotional contexts such as behavioral inhibition and resolution of interference.

\section{Dialectical repression theory}

\author{
David H. Gleaves \\ Department of Psychology, University of Canterbury, Private Bag 4800, \\ Christchurch, 8140, New Zealand. \\ david.gleaves@canterbury.ac.nz \\ http://www.psyc.canterbury.ac.nz/people/gleaves.shtml
}

Abstract: Erdelyi's dialectical repression theory attempts to reconcile what appear to be incompatible perspectives in the contentious area of memory for trauma. He partially succeeds and makes a strong case that repression is "an empirical fact," but makes a weaker case that distortions and omissions are due to the same mechanism and that recovered memories are necessarily unreliable. Available data do not suggest that the return of the repressed is any less accurate than the return of the non-repressed.

Erdelyi's theory of repression in the target article purportedly "integrates the largely dissociated data of the clinic and the laboratory" (sect. 1), the same goal as of the recent paper by Gleaves et al. (2004). Many of his conclusions are similar to ours; others are not. We agree on the obvious, that "Repression has become an empirical fact" (target article, Abstract), and that "the recovery of inaccessible memories has been part of scientific psychology for more than a century" (sect. 4, para. 9). Gleaves et al. have also noted a wealth of experimental data that support the reality of what they term blocked and recovered memories. However, Erdelyi goes on to argue that the return of the repressed is necessarily associated with distortion, and that such memories shouldn't be trusted for accuracy. Here, disagreement arises (at least to a degree), which I address later in this commentary. The common theme in Erdelyi's theory is that omissions and distortions are both due to repression. Thus, if you believe in false memories, you apparently also believe in repression (and vice versa). Given the polarity of the field regarding false and repressed memory, Erdelyi's attempt might better be considered a dialectical theory of repression.

There are many dialectics in the field of memory for trauma such as Widiger and Sankis's (2000) position that "difficulty forgetting (or letting go of) a horrifying experience may simply be the opposite side of the same coin of difficulty remembering (accepting or acknowledging) a horrifying experience" (p. 391). A related dialectic, noted by Erdelyi, is how strong emotion both enhances and degrades memory. However, his discussion of central versus peripheral details only deals with the effects of moderate emotion. At higher levels of stress, amnesia and hypermnesia may occur for different modes of memory. As noted by Jacobs and Nadel (1998), "It [traumatic stress] can cause amnesia for the autobiographical context of the stressful event and hypermnesia for the emotional memories produced by them" (p. 1115). This effect is related to Erdelyi's point that repression "knocks out declarative (conscious) memories, but other memory systems (e.g., procedural ones, as in symptoms) are not similarly affected and continue to reflect remembering" (sect. 4, para. 6). Erdelyi asks if memory stops expressing itself through less direct channels once recovered in the conscious channel, and there are clinical data that suggest this to be the case (Wickramasekera 1994).

Erdelyi moves the field both forward and backward at the same time. He moves it forward by clarifying that the conscious/unconscious distinction allegedly attributed to repression (i.e., that only evidence of an unconscious process counted - see also Gleaves 1996) is a "reconstructive memory distortion operating at the level of the field" (sect. 2, para. 5). As Erdelyi notes, when repression is defined to include conscious or intentional processes, there is no question that it exists, and he is obviously right about that. However, he moves the field backward by overstating the degree to which memory is distorted and subject to reconstruction. This commonly accepted position is not supported by either experimental or clinical data. Erdelyi refers to Wegner's (1989) research demonstrating a rebound effect of thinking about white bears. It is noteworthy that there was not a rebound of pink bears, black bears, or white rabbits. What returned was the same as was initially repressed. In terms of clinical data (not cited by Erdelyi), the only research on the accuracy of recovered versus continuous memories (i.e., those that were never repressed; Dalenberg 1996) suggests that they are equally accurate. Erdelyi also argues that it was also Freud's position that previously repressed material is invariably altered. However, in citing Freud's work, Erdelyi either provides quotes that are out of context (from Freud 1896a/1962), or cites his later work after Freud had abandoned his seduction theory in favour of his fantasy-based reformulation. Regarding the first issue (citing out of context), the quote that "Re-activated memories ... never re-emerge into consciousness unchanged" (sect. 5, para. 10) was from Freud's discussion of obsessional neuroses in which the reactivated (which is not the same as recovered) memory was conjectured to be transformed into the neurotic symptom. This was different from his theory of hysteria in which he did not doubt the accuracy of memories and, in fact, based the theory on the assumption that they were accurate. There is no question that Freud didn't trust unconscious memories in his reformulated theory, and this was allegedly one of his reasons for changing his theory. However, whether or not he was correct in doing so is a different issue (see Gleaves \& Hernandez 1999). Erdelyi simply describes Freud's initial theory as "erroneous" when there is far greater evidence that his reformulated theory was erroneous as well as tremendously harmful. Even supporters of the false memory movement (e.g., Powell \& Boer 1995) argue that Freud subsequently interpreted real memories of abuse as fantasies, and subsequent to that time, when women reported sexual trauma to psychoanalysts, it was interpreted as sexual fantasy.

All memory is subject to error, but according to Bartlett, mental schemas intrude on and distort memory. That being the 
case, intrusions/distortions are likely to be schema-consistent. No false memory research published to date would suggest otherwise. In the Deese paradigm, participants might misremember the word sleep after hearing "bed, rest, awake, tired, dream, wake, and snooze" (target article, sect. 5), but they don't misremember the words rape or incest (see also Freyd \& Gleaves 1996). Although this seems obvious, it doesn't stop some writers (including Erdelyi) from arguing that such data support the current false memory movement. When people mis-remember, what they do remember is likely to be consistent with their existing schemas (Pezdek et al. 1997), or perhaps with their actual experiences. Thus, there continues to be no evidence to support the commonly expressed false memory position that that people who come from non-traumatic family environments can be induced to mis-remember their childhoods as traumatic and abusive. Following Freud's mistakes, reports of abuse were interpreted as fantasy. Now they are routinely interpreted as false memory. The corrective programs (alluded to by Erdelyi) that are really needed are those that will undo such damage.

\section{On the continuing lack of scientific evidence for repression}

\author{
Harlene Hayne, ${ }^{a}$ Maryanne Garry, ${ }^{b}$ and Elizabeth F. Loftus ${ }^{c}$ \\ ${ }^{a}$ Department of Psychology, University of Otago, Box 56, Dunedin, New \\ Zealand; ${ }^{b}$ Victoria University of Wellington, School of Psychology, Box 600, \\ Wellington, New Zealand; ' ${ }^{\mathrm{C}}$ Department of Social Ecology, 2393 II, University \\ of California, Irvine, CA 92697-7085. \\ hayne@psy.otago.ac.nz http://psy.otago.ac.nz/staff/hayne.html \\ maryanne.garry@vuw.ac.nz \\ http://www.vuw.ac.nz/psyc/staff/maryanne-garry/index.aspx \\ eloftus@uci.edu http://www.seweb.uci.edu/faculty/loftus/
}

Abstract: The forgetting and remembering phenomena that Erdelyi outlines here have little to do with the concept of repression. None of the research that he describes shows that it is possible for people to repress (and then recover) memories for entire, significant, and potentially emotion-laden events. In the absence of scientific evidence, we continue to challenge the validity of the concept of repression.

Right off the bat, Erdelyi comes out swinging. "Repression," he declares, "has become an empirical fact that is at once obvious and problematic" (target article, Abstract). Is it an empirical fact in the same way that, say, gravity is an empirical fact? Well, no. We can demonstrate the effects of gravity simply, quickly, and whenever we want; the same is not true of repression. Is repression a widely accepted theory, in the same way that, say, the theory of evolution is? Well, no. Converging evidence from biology, biochemistry, and anthropology supports evolution. This is not true of repression. Is repression an idea that sounds interesting but turns out to be something that nobody can find any evidence for - like, say, cold fusion? Yes. That's more like what repression is: cold fusion. Repression has become the clinician's cold fusion, at once obvious and problematic.

Erdelyi draws on three research findings to argue that repressed and recovered memories are supported by science: We forget things, we remember things, and sometimes, if we try really hard, we remember more things over time. Take, for example, Erdelyi's appeal to studies by Bartlett and Ballard. Yes, subjects remembered more studied material if they attempted to recall it over and over again, but they never forgot that they had encountered the material in the first place. Likewise, patient "B." never forgot having a brother, and never forgot that he had died. These examples tell us a lot about our ability to forget and then remember specific details about a particular event, but they tell us nothing about the wholesale repression of an entire experience, particularly one we can later recall in great detail.
Erdelyi also uses research by Anderson and his colleagues to validate the notion of repression, but what do the Anderson studies actually show? In Anderson and Green (2001), subjects first learned a set of word pairs (such as ordeal, roach). Next, they saw one word from a pair (ordeal) and tried to recall its partner (roach), tried to suppress its partner, or did not see the words from a pair. Finally, subjects took a memory test: they were shown the first word of the pair, and they tried to recall the second word. The crucial question was whether subjects would be less likely to report words they had tried to suppress compared to words they had not studied in the second phase (baseline). Anderson and Green found that sometimes being asked to suppress the word impaired memory for it. They concluded that their results "support a suppression mechanism that pushes unwanted memories out of awareness, as posited by Freud" (Anderson \& Green 2001, p. 368).

But a closer look at these findings shows us that whatever mechanism was operating, it was not pushing many memories anywhere. Even after 16 suppression attempts, people still recalled the words about $75 \%$ of the time. Although this recall rate was below baseline (about 83\%), it wasn't overwhelmingly lower, and, more to the point, we assume that none of the subjects had forgotten that they had participated in the experiment in the first place. Once again, research on the forgetting of small bits of information tells us nothing about repression of entire events.

More recently, Anderson et al. (2004) used fMRI to measure subjects' brain activity during the phase when they were recalling or suppressing. Different patterns of brain activity occurred when subjects tried to recall words than when they tried to suppress them. For example, when subjects tried to remember words, they showed more activity in the hippocampus; when they tried to suppress words, they showed more activity in other regions. This is not support for "repression." Rather, brain activity in other regions undoubtedly reflects subjects' attempts to think about something else, shuttling cognitive resources elsewhere.

It is also worth noting that others have not replicated Anderson's findings, despite several careful attempts to do so (Bulevich et al., in press). Even if we assume the suppression effect does occur, it seems small and unreliable. Erdelyi argues that the architecture of repression is agnostic to content - that we must distinguish between the mechanism and the reason the mechanism is unleashed. Yet, if Freud himself had described a mechanism that interferes with recall only $8 \%$ of the time, he would have been a footnote in the history of science, and none of us would have lived through the memory wars.

Erdelyi describes decades' of research on memory distortions as supporting Freud's view about the reconstructive nature of memory; he tells us that Freud knew that memory was reconstructive. Yet Erdelyi omits the relatively recent work showing just how extreme this reconstructive process can be. In the past decade, nine published studies conducted with adults reveal a $33 \%$ reporting rate for rich false memories for madeup experiences ranging from spilling punch at a wedding, to being attacked by an animal, to riding in a hot air balloon (Strange et al. 2005); the false memory rate for children is even higher (Gross et al. 2006). Freud never anticipated this capability of the human mind, or the role of therapy in helping to construct such rich false memories (Loftus \& Bernstein 2005).

Ironically, for all Erdelyi's insistence that we could have spared ourselves a century of wrongful convictions and divided families if only we had listened to Freud, we find ourselves thinking the same thing about psychological science: If only people would listen. Many years ago, when scientists found that post-event information could alter memories for details, many skeptics viewed the findings as neat laboratory "tricks," but questioned their clinical or legal significance. When wrongfully convicted people were exonerated by DNA analysis, some skeptics stopped criticizing and started listening. Scientists tackling 
more recent problems have endured similar accusations (Loftus \& Guyer 2002a; 2002b; Roediger \& McDermott 1996). Eventually, when those scientists began to show that it was possible to implant memories for entirely false events, a few people in the clinical and legal community started listening. Every day brings a few more. Those who advocate a mechanism that represses and recovers memories should adhere to the same high standard. Listen to the science.

\section{Reduced autobiographical memory specificity, avoidance, and repression}

\section{Dirk Hermans, ${ }^{\text {a }}$ Filip Raes, ${ }^{\text {a }}$ Carlos Iberico, ${ }^{\text {a }}$ and J. Mark G. Williams ${ }^{b}$ \\ ${ }^{a}$ Department of Psychology, University of Leuven, 3000 Leuven, Belgium; ${ }^{\mathrm{b}}$ Department of Psychiatry, University of Oxford, Warneford Hospital, Oxford, OX3 7JX, United Kingdom. \\ dirk.hermans@psy.kuleuven.be http://ppw.kuleuven.be/leerpsy/dirk/ fillip.raes@psy.kuleuven.be carlos.iberico@psy.kuleuven.be \\ mark.williams@psych.ox.ac.uk}

Abstract: Recent empirical work indicates that reduced autobiographical memory specificity can act as an avoidant processing style. By truncating the memory search before specific elements of traumatic memories are accessed, one can ward off the affective impact of negative reminiscences. This avoidant processing style can be viewed as an instance of what Erdelyi describes as the "subtractive" class of repressive processes.

Erdelyi conceptualizes repression broadly as a class of operations that reduces the accessibility to consciousness of some target material. According to Erdelyi's "unified theory of repression," there are two ways in which this goal can be accomplished. The first consists of processes that add noise to the signal ("elaborative or additive processes"), examples of which are denial and rationalisation. The second are processes that lower the accessibility by degrading the signal ("inhibitory or subtractive processes"). Erdelyi discusses empirical work that supports both types of repression. However, an important series of studies, though missing from his overview, may be consistent with, and further elaborate, his conclusions. We refer here to recent work on reduced autobiographical memory specificity (for an overview, see Williams et al., in press), which provides a clear demonstration of a class of "subtractive processes."

Autobiographical memory specificity refers to the cognitive capacity of accessing memories of personally experienced past events in a specific way. This capacity is typically assessed using the Autobiographical Memory Test (AMT; Williams \& Broadbent 1986). The AMT consists of the presentation of a series of cue words (e.g., happy, sad) for which participants are asked to respond, typically within 30 seconds, with a personal event that the word reminds them of. The event retrieved should be specific; something that happened at a particular place and time and lasted for a day or less. For instance, a specific response to the cue word "happy" would be "Last Friday evening when my daughter phoned me to say that she won a prize at school."

The ability to be specific in the retrieval of autobiographical information is of crucial importance for our day-to-day functioning. For example, it allows us to benefit from previous experiences in the effective pursuit of current goals (e.g., Goddard et al. 1996). In the context of the present discussion, however, it is important to note that there is clear evidence that reduced autobiographical memory specificity (1) can be observed in persons who experienced a history of trauma, and (2) can be functional in diminishing the accessibility of unpleasant/aversive memories.
During the last two decades, memory specificity has been studied in various populations. It has become increasingly clear that reduced memory specificity is particularly characteristic of two groups of persons: those suffering from major depression, and those who report a history of abuse or other traumatic adversities (Williams et al., in press). In spite of repeated prompting, both groups respond with significantly fewer specific memories to the cue words of the AMT, and produce more "overgeneral" memories. The latter are most often memories that refer to categories of events (e.g., "I feel happy whenever I visit my parents"), rather than to specific occurrences. At present, more than a dozen studies have confirmed the association between trauma and reduced specificity (Hermans et al. 2004). The findings range from emotional abuse (e.g., de Decker et al. 2003) and physical abuse (e.g., Hermans et al. 2004), to sexual abuse (e.g., Henderson et al. 2002; Kuyken \& Brewin 1995).

An explanation for this association is that reduced memory specificity is a functional strategy that diminishes the accessibility to consciousness of trauma-related memories. By truncating the memory search before accessing specific representations, one can avoid the confrontation with sensory and perceptual fragments of an event that, if activated, might produce large and catastrophic increases in mood disturbance. As a result, a more "overgeneral" retrieval style might develop due to negative reinforcement (i.e., the non-confrontation with the emotionally arousing specificities of the trauma). This strategy will be adaptive only if it is not restricted to trauma-related memories. Because the associative pathways of human memory are often rather unpredictable, this avoidant strategy can be successful only if it is not selective and pertains to memory in general.

One type of evidence for the idea that reduced memory specificity can act as an avoidant coping style is correlational. Hermans et al. (2005) have demonstrated that the number of specific responses on the AMT correlates significantly with several measures of avoidant coping. The less specific, the more avoidant the participant. Similar results have been described by other authors (e.g., Kuyken \& Brewin 1995).

Other evidence stems from recent experimental work. In a study that compared high- and low-specific participants, Raes et al. (2003) found that the degree of mood disturbance following the experimental manipulation of stress was significantly less in low-specific participants. These results illustrate the possible pay-off for people with a less specific retrieval style: They are less emotionally aroused by a negative personal experience. This finding was recently replicated (Raes et al. 2006, Experiment 1).

The whole of these findings strongly suggests that reduced autobiographical memory specificity can act as way to reduce the accessibility to consciousness of negatively valenced or traumatic memory traces. In this sense it can be regarded as a form of "repression" as defined by Erdelyi. Against this background, it is important to note that Raes et al. (2006, Experiment 1) have shown that reduced memory specificity correlates significantly with Mendolia's index of repression (Mendolia 2002), clearly indicating that the more a repressive coping style is used, the less specific autobiographical memories are retrieved. Similar results were obtained by Blagov and Singer (2004), who found a correlation between repressive defensiveness and memory specificity, with higher defensiveness being associated with fewer specific memories recalled (also see Dickson \& Bates 2005).

In conclusion, we believe that the extensive literature on autobiographical memory specificity (at present more than 150 published manuscripts) is highly relevant to the discussion of the repression concept. In Erdelyi's distinction between additive and subtractive processes, reduced specificity can be placed within the latter category (in the sense of "eliminating thinking" about some material). Whether or not this form of avoidant coping is based on conscious processes, is a topic for further research. However, we do not exclude the possibility that it can be shaped by negative reinforcement without the need for conscious monitoring. 


\section{Repression: A unified theory of a will-o'-the- wisp}

John F. Kihlstrom

Department of Psychology, University of California, Berkeley, Berkeley, CA 94720-1650.

jfkihlstrom@berkeley.edu http://socrates.berkeley.edu/ kihlstrm

Abstract: By conflating Freudian repression with thought suppression and memory reconstruction, Erdelyi defines repression so broadly that the concept loses its meaning. Worse, perhaps, he fails to provide any evidence that repression actually happens, and ignores evidence that it does not.

Erdelyi's "unified theory of repression" is his latest attempt to resuscitate this most central concept within Freud's theory of psychoanalysis (Erdelyi 1985; 1990; 1993; 1996; Erdelyi \& Goldberg 1979). Unfortunately, the present effort is no more successful than the earlier ones.

Erdelyi gets off to a poor start by attempting to show that Freud defined repression broadly to include the conscious suppression of memories, meaning that the act of repression can itself be performed consciously, so long as the repressed mental contents themselves are denied access to consciousness. While it is true that "Freud used repression and suppression interchangeably" (target article, sect. 2, para. 5), Erdelyi's own tabulation (Erdelyi 1990, pp. 9-10) shows that this equation occurs primarily in Freud's earliest writings (see also Esterson 2003). It may well have been Anna Freud (1936/1937) who ultimately dictated that repression must always be unconscious. But apparently by the time psychoanalytic theory had matured, Sigmund Freud himself (Freud 1915a/1957; 1915b/1957) appears to have understood that repression must be unconscious - not just if the process is to have any chance of working, but also if the concept is to make any sense at all (for a thorough analysis of the vagaries of Freud's concept of repression, see Macmillan 1991/1997).

Erdelyi fixates on Freud's earliest, and least coherent, concept of repression because his argument fails without it. As he states, "If repression = suppression, then everybody believes in repression" (sect. 2, para. 7). Maybe, but not so fast. For example, Anderson and his colleagues (Anderson 2001; Anderson \& Green 2001; Levy \& Anderson 2002), among others (Conway 2001; Gleaves et al. 2004; Smith et al. 2003), have argued that deliberate retrieval inhibition, a variant on directed forgetting (Bjork 1978; Epstein 1972; Kihlstrom \& Barnhardt 1993), is a viable laboratory model for the clinical repression of traumatic memories. Erdelyi cites this work favorably, even though its relevance to repression has been vigorously disputed (Kihlstrom 2002; 2004; 2006; Schacter 2001; for a reply, see Anderson \& Levy 2002). Setting aside the issue of whether repression can indeed be conscious, the to-be-forgotten material in the retrieval inhibition experiments is not traumatic, conflict-laden, or anxiety-evoking. Even extensive practice with retrieval inhibition fails to produce anything even remotely resembling amnesia. And there is no evidence of the return of the repressed material in the form of implicit memories and other "symptoms." Put bluntly, the analogy is baseless - even more so, if repression does not equal suppression, and repression must be unconscious after all.

Erdelyi similarly overreaches when he offers his and others' own research on reminiscence effects and hypermnesia (e.g., Erdelyi 1996) as corroboration for "Freud's claim" (sect. 4, para. 7) that unconscious memories may be recovered with concentration and repeated effort. But we did not need Freud to tell us that people remember better when they try harder. In any event, none of the stimulus materials in the hypermnesia experiments even approached traumatic significance; and none of the forgetting from which the subjects recovered was motivated, either consciously or unconsciously, by considerations of defense. Like retrieval inhibition, hypermnesia as studied in the laboratory is simply irrelevant to the Freudian concept of repression.

For that matter, so is Bartlett's (1932) work on reconstructive processes in memory. While it is true that some of Freud's supplementary defense mechanisms, like rationalization and symbolization, have their cognates in Bartlett's list of memory distortions (target article, Table 1), any equation between the two strips both Bartlett's and Freud's concepts of all their meaning. Erdelyi writes, "The constructions and reconstructions of Freud and Bartlett are the same but for motive" (sect. 5, para. 2). But since motive is everything in Freud - whether the sexual and aggressive motives of the id or the anxiety-reducing motives of repression and the other defense mechanisms of the ego - they are not the same at all. From "The War of the Ghosts" to The Psychopathology of Everyday Life is a "chancy leap" (sect. 5, para. 3) indeed; let's just not go there.

Retrieval inhibition, elaborative reconstruction, and hypermnesia might serve as mechanisms for repression and the recovery of lost memories, but the target article ignores the most important question of all - which is, whether there is any empirical evidence for repression in the first place. On that score, all we get is the reassurance, in the Abstract, that repression is an "obvious" "empirical fact," plus two "clinical fragments" - B.'s conscious suppression (sect. 3.2.1) and N.'s paranoid delusion (sect. 3.3). Erdelyi does refer to "the contentious area of memory for trauma” (sect. 3.2.1.1, para. 2) - but without ever confronting the fact that dozens of formal studies have yielded not a single convincing case of repression in the entire literature on trauma (see, e.g., Kihlstrom 2006; McNally 2003; Pope et al. 1999). Apparently, most traumatized individuals remember their traumas all too well; and where trauma is forgotten, it appears to be by virtue of processes other than repression.

Erdelyi's first mistake, and it is a big one, is that he defines repression so broadly as to strip the concept of all the features that might make it interesting. As a result, the unification he achieves is entirely Procrustean: the only way the elements can all be fit together is to so severely distort each one of them that they become unrecognizable. His second mistake, and it is equally big, is to ignore the actual empirical evidence about trauma and memory. The result is a unified theory of nothing at all.

\section{Universal repression from consciousness versus abnormal dissociation from self- consciousness}

\section{Robert G. Kunzendorf}

Department of Psychology, University of Massachusetts Lowell, Lowell, MA 01854.

Robert_Kunzendorf@UML.edu

\begin{abstract}
Freud attributed uncovered incest, initially, to real abuse dissociated from self-consciousness, and later, to wishes repressed from consciousness. Dissociation is preferred on theoretical and empirical grounds. Whereas dissociation emerges from double-aspect materialism, repression implicates Cartesian dualism. Several studies suggest that abnormal individuals dissociate trauma from self-conscious sourcemonitoring, thereby convincing themselves that the trauma is imaginary rather than real, and re-experience the trauma as an unbidden image.
\end{abstract}

It is unfortunate that Erdelyi's effort to unify clinical and laboratory data ignores the century-old dispute between Freudian theorists who attribute clinical data to a universal dynamic of repression from consciousness and other psychodynamic theorists who attribute clinical data to a pathological process of dissociation from self-consciousness. The dissociative approach was originally developed by Freud's contemporaries - Janet (1907; 1910) and Prince $(1910 ; 1925)$ - and was philosophically grounded on Fechner's (1860/1966) double-aspect materialism. 
In opposition to the Cartesian conception of mind as a nonphysical homunculus that is "conscious of" both sensory material and itself, Fechner conceived the mind to be a bundle of conscious sensations - where particular sensations are subjective aspects of particular neural events, and where such events are not always above the limen or threshold for innervating the illusion of self-consciousness. Extending Fechner's framework to clinical theory, Prince (1910) articulated the conceptual basis for dissociation:

If it is true that dissociated brain systems can functionate ... and if it is true that they have psychical equivalents, then whether we are selfconscious of any given state of consciousness must depend ... upon whether the brain process, correlated with it, is synthesized in a particular way with the larger system of brain processes which is correlated at a given moment with the self-conscious personality. And in so far as a brain process can occur detached from the main system of brain processes, so far can consciousness occur without self-consciousness. (p. 94)

Prince (1925) further extended this theoretical framework to the interpretation of clinical data:

The introspective testimony of my dissociated subjects, who in [hypnosis] recalled vividly and precisely these subconscious experiences [of trauma], has been unanimous that these experiences were without self-consciousness... The conscious events were just sensations. (p. 182)

Freud's early approach to hypnotically uncovered traumas, like Prince's approach, presumed them to be real traumas that had become dissociated from self-consciousness. But later, after incestuous trauma was uncovered in his own therapy, Freud reattributed such uncoverings to universal wishes which, not only in patients but also in "normal" people, are repressed from consciousness (Davis 1990). And in order to accommodate the repression of universal wishes, Freud (1916/1922) abandoned double-aspect materialism for a new version of Cartesion dualism:

The unconscious system may therefore be compared to a large anteroom.... Adjoining this is a second, smaller apartment, a sort of reception-room, in which consciousness resides. But on the threshold between the two there stands a personage with the office of door-keeper, who examines the various mental excitations, censors them, and denies them admittance to the reception-room when he disapproves of them.... The excitations in the unconscious, in the ante-chamber, are not visible to consciousness, which is of course in the other room, so to begin with they remain unconscious. When they have pressed forward to the threshold and have been turned back by the door-keeper, they are "incapable of becoming conscious"; we call them then repressed. But even those excitations which are allowed over the threshold ... only become [conscious] if they succeed in attracting the eye of consciousness. (pp. 305-306)

What is new about Freud's version of substance dualism is that it posits two homunculi: the "door-keeper" and the "eye of consciousness."

Freud's (1900/1913) Interpretation of Dreams supposedly provided symbolic evidence for the universality of incestuous wishes "in the ante-chamber." However, laboratory studies have demonstrated that the second level of meaning in a "symbolic" dream arises from lingering emotions that consciously accompany the dream, not from unconscious wishes (Hartmann et al. 2002-2003; Kunzendorf \& Maurer 1988-1989; Kunzendorf \& Paroskie 2000-2001; Kunzendorf et al. 1999-2000). Thus, Hartmann (1998) describes rape victims who have complete memories of their traumas, without any signs of repression and who also have "symbolic" dreams of ocean waves that become overwhelming tidal waves, as their dream images begin to contextualize their consciously lingering feelings of being overwhelmed.

Recently, the non-Freudian notion of dissociation from selfconsciousness has been clinically revived by Spiegel (1986; 1990) and Bliss (1986; 1988), and has been theoretically refined by
Kihlstrom and Cantor (1984) and Kunzendorf (2000). According to Kunzendorf, one's illusory experience of a self-conscious subject "having" sensations is subjectively manifested as either the tacit knowledge that one is "imaging" sensations or the sensationless knowledge that one is "perceiving" them. Furthermore, the tacit knowledge that one is imaging is the subjective aspect of a neural mechanism monitoring the central innervation of imaged sensations, and the tacit knowledge that one is perceiving is the subjective quality of the same mechanism monitoring the peripheral excitation of perceived sensations.

On Kunzendorf's account, abnormal individuals attenuate self-conscious source-monitoring not only during sleep but also during trauma, thereby encoding the trauma as an imaginary or dreamlike experience, and ultimately remember the trauma not as a real happening but only as an unbidden image. In research confirming this source-monitoring account of dissociation, Kunzendorf and Karpen (1996-1997) demonstrated that the dissociative experience of amnesia is associated with attenuated source-monitoring and, employing Holmes' (1990) paradigm, Kunzendorf and Moran (1993-1994) further demonstrated that traumatic stress induces "dissociation from self-conscious source-monitoring" and source amnesia, rather than "repression from consciousness" and content amnesia. Moreover, the return of dissociated trauma - the unbidden image - is contingent on neither free association as posited by Freud nor hypermnesia as posited by Erdelyi, but on shifting one's recall criteria from "previously perceived" sensations to "familiar" (perceived or imaged) sensations (Kunzendorf 1989-1990; Kunzendorf \& Boisvert 1996).

Finally, Erdelyi's distinction between "repression from consciousness" and "distortion of consciousness" misses the important source-monitoring relationship between dissociation and hallucination. In persons whose wakeful source-monitoring is unstable and unreliable, dissociative symptoms tend to emerge when the realities of abuse are defensively inferred to be nightmarish imaginings instead of traumatic percepts, and psychotic symptoms tend to emerge when mental images of the taboo are defensively inferred to be percepts beamed into the head by the devil or the C.I.A. instead of self-generated images. Indeed, among persons subjected to extreme childhood abuse and diagnosed with dissociative identity disorder (Kluft 1987a), Kluft observed first-rank symptoms of delusion and hallucination in 30 out of 30 cases (Kluft 1987b). However, neither dissociative inferences about traumatic percepts nor psychotic inferences about taboo images are likely to arise in persons whose wakeful source-monitoring is robust: Kunzendorf et al. (1997-1998) observed dissociative symptoms primarily in those abused patients who scored high on Tellegen and Atkinson's (1974) test for Absorption in Fantasy - a test which Rader et al. (1996) found to be predictive of unstable source-monitoring.

\section{Repression and the unconscious}

\section{Robert Langnickel and Hans Markowitsch \\ Department of Physiological Psychology, University of Bielefeld, 33501 Bielefeld, Germany. \\ robert.langnickel@philosophie.uni-bielefeld.de \\ hjmarkowitsch@uni-bielefeld.de \\ http://www.uni-bielefeld.de/psychologie/ae/AE14/HOMEPAGE/ \\ Markowitsch_home.html}

Abstract: We argue that repression is primarily an unconscious process and that the position of Erdelyi is not coherent with Freud's views on the matter. Repression of ideas is a process that takes place without the knowledge of the subject. In this respect, it is essentially different from suppression, where ideas are acted upon by a conscious will.

Erdelyi rightly emphasizes that there is a "Babel of misunderstandings" (target article, Abstract) in psychology about the 
notion of repression. Therefore, we should welcome the attempt he makes in his "unified theory of repression" to clarify this notion and to integrate different experimental results into the theory. Nevertheless, Erdelyi's account has serious difficulties of its own, especially concerning the relationship between repression and the role of the unconscious.

In leveling out the difference between suppression and repression, Erdelyi neglects the fundamental distinction between conscious and unconscious processes, which is essential for repression. Freud wrote that "repression [...] cannot arise until a sharp cleavage has occurred between conscious and unconscious mental activity" (Freud 1915a/1957, p. 147) and added that there had to be a strong connection between repression and what is unconscious (Freud 1915a/1957, p. 148).

Freud assumed a hierarchy of defense ("Abwehr"), reaching from the most basic defense mechanisms - such as flight from external danger - up to highly sophisticated mechanisms such as judgments of condemnation. Repression stands between these extremes; the only difference is that it is not directed at an external, but at an internal source of danger - an instinctual impulse, whose satisfaction would be incompatible with other demands of the mental apparatus. Repression is an instinctual vicissitude (Freud 1915a/1957, p. 148); and instincts or instinctual representatives also constitute the core of the unconscious (Freud 1915b/1957, p. 185). This relationship is ignored by Erdelyi; the connection between repression and instinctual impulse remains unclear.

According to Freud, there are three stages of repression: primal repression, repression proper (or after-pressure), and the return of the repressed (Freud 1915a/1957, pp. 148, 154). In the first stage of repression, the instinctual representative is barred from entering into the conscious realm, and a fixation in the unconscious takes place. With primal repression, an attraction pole for further repressions is created, where neither the content of the repressed idea nor the attraction itself is conscious to the subject. Unfortunately, Erdelyi does not take this into account. The after-pressure - the second stage of repression - is connected in an associative manner with what was primarily repressed; the primal repression provides a path for the repression proper and precedes it logically (Freud 1915a/1957, p. $148 ; 1926 \mathrm{a} / 1959$, p. 94 ).

In his Metapsychology, Freud holds the view that an idea can only be repressed if it is already attracted by unconscious contents. In this way, he presupposes an unconscious, which - as the attracting force - cannot itself be something that has been attracted. The unconscious therefore seems to be the product as well as the precondition of individual repression. The construct of primal repression is supposed to dissolve this seeming contradiction in providing a starting point. In the third stage, a "return of the repressed"may happen. Repression is a precondition of symptom formation (Freud 1916-1917/1961, p. 294); the breach - "the return of the repressed" - occurs at the point where a fixation has taken place in the past. This is the reason why repression is also regarded as a main factor for the development of psychoneuroses (Freud 1905/1958, p. 101), dreams, and parapraxes.

But Freud not only specifies the result of repression as unconscious and describes the repressed idea as inapt for consciousness (Freud 1916-1917/1961, p. 296), he also characterizes the process of repression as something that happens without being noticed by the ego (Freud 1916-1917/1961, p. 294). Erdelyi neglects this aspect. In the second topic model of the mental apparatus, which Freud developed between 1920 and 1923, the unconscious is no longer presented as a closed system, but rather as a constituent of all three instances (id, ego, and superego), controlling one of the instances - the id - completely. This explains why the repressed is associated with the ego, but only with the unconscious part of it (Freud 1923/1961, p. 18, 24). The conceptual confusion which may be caused by talk of repression by the ego, can be dissolved if we notice that only the unconscious part of the ego is supposed to be doing the repressing
(Freud 1933/1964, p. 69), and that there is no conscious process of repression by the ego, contrary to what Erdelyi postulates.

The strength of the "unified theory of repression"depends on how exactly repression is defined. When repression is defined as an intentional suppression of an experience, then there is no controversy, and Erdelyi is right when he states, "If repression = suppression, then everybody believes in repression. The myth morphs into the obvious" (sect. 2). But when we construe repression as something that operates unconsciously, refers to instinctual representatives, and presupposes a primal repression attracting instinctual representatives, then Erdelyi has not presented an integrating account, but instead an account that excludes Freud's position.

The proposed unified theory of repression is not a theory of repression at all; the conception of the Freudian unconscious has no place in it, and neither has psychoanalysis, which is the science of the unconscious, according to Freud (cf. Freud $1926 \mathrm{~b} / 1959$, p. 265). The "unified theory of repression"leads in the last instance to the identification of the mental with the conscious - a view that has been firmly rejected by Freud (cf. Freud 1916-1917/1961, p. 22).

\section{Is Erdelyi's swan a goose?}

\section{Malcolm Macmillan}

\section{Department of Psychology, School of Behavioural Science, University of Melbourne, Melbourne, Victoria 3010, Australia. \\ malcolm.macmillan@unimelb.edu.au}

Abstract: Erdelyi's argument for integrating clinical data on repression, mainly Freudian, with laboratory findings suffers from logical and factual errors concerning Freudian repression and about experiments on forgetting.

Erdelyi's argument for integrating clinical data on repression, mainly Freudian, with laboratory work on forgetting is marred by a serious logical flaw: that is, by his virtually restricting consideration of Freudian repression to the initiating motive, by his avoiding primary and secondary repression, and by misunderstandings of the work on forgetting, especially that of Ebbinghaus and Bartlett.

First, regarding the logical problem: Erdelyi cites Freud's saying that the essence of repression lies in keeping something out of consciousness. But it does not follow that everything that is kept out of consciousness is kept out because of repression. It's the old problem of the white swan: Because all swans are white birds, and this bird is a swan, it certainly follows that this bird must be white, but it is fallacious to assert that any given white bird is a swan. The similarity between repression and forgetting proposed by Erdelyi is based on such a fallacy.

Second, regarding the restriction to motive: As Erdelyi says, Studies on Hysteria (Breuer \& Freud 1895/1955) contains many instances of Freud's patients telling him they had deliberately tried to keep some unpleasant memory or thought out of consciousness. But that is only the beginning of the story. In what Freud initially called defence hysteria - soon the only form of hysteria he recognised - an initial effort of will brought about a split in consciousness in which symptoms formed. The patient did not intend to cause the split and generate symptoms - his intention was different but unsuccessful (Freud 1894/ 1962 , pp. 46-47). Thus, an obsessional patient said, "I tried to put it away from me and tried not to think about it ... but then I got this other thing" (i.e. the symptom) (Freud 1894/1962, pp. 52-53). Freud said quite explicitly that he hoped his theory would fill the gap between the act of will and the emergence of the symptom. His central theoretical proposal was the separation of the sexual idea from its charge of affective energy. In hysteria, the affect was converted into motor or sensory symptoms; and in obsessions, it was attached to or 
displaced on to another idea. Repression thus came after the act of will. Freud emphasised that the processes coming after the act of will and before the appearance of the symptom "occur without consciousness" and can "only be presumed, but cannot be proved by any clinico-psychological analysis” (Freud 1894/1962, p. 53).

Freud had to, of course, make other inferences about repression. Why was it that only sexual ideas were repressed? The short answer is so dismissed by Erdelyi as an isolated and ambiguous titbit that one sees he has missed its significance: the pull into the unconscious from the repressed contents already there. Simplifying the longer answer, we can say Freud's conceptualisation passed through two stages. First he postulated that the pull in hysteria came from repressed early sexual experiences of an unpleasant kind, and from pleasant ones in obsessions. Later, as part of the extension of his theses on childhood sexuality, he had the pull coming from repressed fantasies based on the erogenous zone components of a perverse childhood sexual drive. Where does Erdelyi recognise this process?

Third, regarding the comparison of Erdelyi's conceptions of repression with laboratory work: The experiments that Erdelyi cites or refers to mainly concern the effects of actively trying to forget an idea, or of the effects of repeated recall. None of the material used in these experiments is especially "unacceptable" to the ego or even particularly affect-laden. Consequently, they are only slightly relevant to Erdelyi's "clinical repression" and not at all to Freud's concept. Further, Erdelyi's parallel between the rebound effect in the "White bear"-type experiments and Freud's "return of the repressed" is misleading because the main thing that returns is the compromise formation that is the symptom - not the original unacceptable idea on which the symptom is partly based.

Turning now to Ebbinghaus (1885/1964), we find that he says nothing about not thinking about the lists of syllables that were to be tested after the longer intervals. Ebbinghaus had not, as Erdelyi puts it, "intentionally excluded" those lists from consciousness between leaning and recall. Any similarities among the kinds of changes discernable in Ebbinghaus' data and those of Erdelyi and Kleinbard are thus of little significance for Erdelyi's unified concept.

The same point holds for Bartlett (1932), who found exactly the same kinds of distortions in tachistoscopic perception, in responses to inkblots, and in reproductions of simple pictures. Most significantly, Bartlett found the same distortions in both repeated and serial reproduction of drawings and passages of prose - significant because in serial reproduction, the reproductions were made by different individuals after having been passed to them by another subject. One cannot sensibly invoke individual repression-like processes in that kind of social transmission. All the distortions over the range of mental content Bartlett investigated were caused by the simple process of being fitted into an existing mental representation (roughly Bartlett's schema), and had nothing to do with repression.

Finally, regarding the "intersection" of Bartlett with Freud, the similarities are either not there or are misleading. Thus, Bartlett's (1932) condensation was simply a shortening (p. 184), but Freud's was a fusing of two or more images and their associated quotas of psychic energy; generally Bartlett's rationalisation (pp. 84-89) robbed reproductions of their puzzling elements, whereas for Freud it was a conscious cover for repressed motives; and nothing like Bartlett's double reversal ( $\mathrm{p}$. 168) - returning to the original - is found in Freud, even though his reversal sounds similar. Of the misleading comparisons, elaboration is the most striking: for Bartlett (1932, pp. 179-85) it was the subject's attempt to match material to an existing model, whereas for Freud it was the dreamer's attempt to overcome the dream's apparent absurdity.

Here we see a good example of what Wittgenstein called the charm of Freud's theorising, a charm deriving from the similarity of his concepts of unconscious processes to those of the conscious counterparts with which everyday life makes us familiar. Perhaps this charm explains the difference between Erdelyi's and Freud's accounts of Freud's "counterwill" patient. For Erdelyi, the patient was in conflict because of wishing and not wishing to feed her baby, whereas Freud's conceptualisation used only an opposition between the very abstract and less readily understandable notions of will and counterwill (Freud 1892-1893/1966). As I have shown (Macmillan 1997, pp. 87-88, 154-155), counterwill was a transitional mechanism, formulated, so to speak, on Freud's journey to a wishful one. Like his conceptualisation of repression, Erdelyi's wishes are here invested with more than a touch of charm.

Overall, it seems that rather than discovering the swan of Freudian repression, Erdelyi has found only a goose.

\section{Let Freud rest in peace}

Richard J. McNally
Department of Psychology, Harvard University, Cambridge, MA 02138.
rjm@wjh.harvard.edu

Abstract: Erdelyi's version of repression - trying successfully not to think about something - is no longer recognizably Freudian. Erdelyi fails to cite directed forgetting experiments involving psychiatric patients that indicate that the motivation to forget threatening material seldom translates into an ability to do so. The early Freud of the seduction theory of hysteria did inspire the recovered memory fiasco.

Erdelyi is among the dwindling minority of experimental psychologists who still take Freud's work seriously. Even those who study emotional processing in psychiatric patients seldom cite Freud. Indeed, the classic text in the field of cognition and emotion references 1,314 books, articles, and chapters, but only one publication by Freud (Williams et al. 1997). Clearly, the field has flourished without him.

Erdelyi, however, believes that contemporary research vindicates Freud, especially his concept of repression. But to render repression palatable to cognitive scientists, Erdelyi whittles it down to its bare bones essence: trying successfully not to think about something. By doing so, he deprives it of its distinctive psychoanalytic character (Crews 1995, p. 272). Freud did not earn his reputation as a bold and original thinker by blandly affirming that people sometimes try not to think about unpleasant things. He earned it by making all kinds of wild claims about what gets repressed, such as the desire of every little boy to murder his father and have sex with his mother. What distinguishes Freud's repression is its connection to other concepts in the system of psychoanalysis, and this system, as Macmillan (1997) has shown, is a conceptual and empirical mess. If repression, plucked from the morass of psychoanalytic theory, is to mean nothing more than trying not to think about something unpleasant, then why bother mentioning Freud at all? We can study motivated forgetting without dragging in the discredited conceptual baggage of psychoanalysis.

Erdelyi discusses research on motivated (directed) forgetting as relevant to Freud's repression. The studies he cites concern normal college students who try to forget neutral material devoid of personal emotional significance. Surprisingly, he does not mention similar studies involving psychiatric patients who endeavor to forget material related to threat or trauma (McNally 2005). Operating from Freudian premises, one might assume that the magnitude of forgetting would be much more pronounced for these individuals who are highly motivated to block this material out of awareness. That is, motivation to forget would seemingly increase one's ability to do so.

But research has shown that this is rarely the case. With two exceptions (Moulds \& Bryant 2002; 2005), psychologists have consistently failed to confirm this "repression" hypothesis. In these studies, psychiatric patients either fail to exhibit superior ability to forget threatening material, or they exhibit outright impairment 
in the ability to forget it. Disconfirming studies include ones on subjects with panic disorder (McNally et al. 1999), obsessive-compulsive disorder (Wilhelm et al. 1996), posttraumatic stress disorder associated with childhood sexual abuse (CSA; McNally 1998), depression (Power et al. 2000), and borderline personality disorder (Korfine \& Hooley 2000). Even adults who report histories of having "repressed and recovered" their memories of CSA fail to exhibit the predicted superiority for forgetting trauma-related material (Geraerts et al. 2006; McNally et al. $2001 ; 2004 ; 2005)$. Merely because individuals are highly motivated to forget something does not mean that they will be able to do so. Indeed, attempts to push unwanted thoughts out of awareness often backfire, enhancing their accessibility (e.g., Beck et al. 2006; Shipherd \& Beck 1999; Wegner 1994).

Erdelyi attempts to exonerate Freud from the charge of having fathered recovered memory therapy. His eagerness to do so is understandable; this therapeutic approach was the worst catastrophe to befall the mental health field since the lobotomy era. But in his plea for Freud's acquittal, Erdelyi misunderstands one key fact: It is not the later Freud of classical psychoanalysis who inspired the recovered memory fiasco; it is the early Freud (1896b/1962) of the seduction theory of hysteria who did so (Crews 1995, pp. 191-223). Nearly every feature of recovered memory therapy can be traced to Freud's early work. In his 1896 article on the "aetiology of hysteria," Freud asserted that repressed memories of childhood sexual abuse are the source of his patients' symptoms, and that these memories had to be recovered and emotionally processed for healing to occur. It is little wonder that the primary theorists of "massive repression" of incest memories (Herman \& Schatzow 1987, p. 12) described Freud's (1896b/1962) article as "brilliant” (Herman, 1992, p. 13). Finally, contra Erdelyi, Freud's (1896b/1962) patients did not "recollect" sexual abuse memories; Freud interpreted their symptoms as indicating repressed memories of molestation, and he relentlessly tried to foist this interpretation on his patients, as Esterson (1993; 1998; 2001; 2002a; 2002b) has convincingly shown.

Uncritical admiration for Freud abounds in 2006, the 150th anniversary of his birth. Not only have his occasional remarks about the brain earned him praise for envisioning cognitive neuroscience, even psychotherapeutic approaches developed in direct opposition to psychoanalysis, such as cognitive therapy, have somehow been traced to his genius. Informed observers realize that these accolades are seldom warranted (for a review, see Crews 2006). Research on motivated forgetting can best proceed unencumbered by Freud's problematic legacy. Freud is dead. Let him rest in peace.

\section{Learning from repression: Emotional memory and emotional numbing}

Nick Medford and Anthony S. David

Division of Psychological Medicine, Institute of Psychiatry, DeCrespigny Park, London SE5 8AF, United Kingdom.

n.medford@iop.kcl.ac.uk http://www.iop.kcl.ac.uk

a.david@iop.kcl.ac.uk

http://www.iop.kcl.ac.uk/iopweb/departments/home/

?locator $=911$ \&context $=$ main

Abstract: Erdelyi argues persuasively for his unified theory of repression. Beyond this, what can studying repression bring to our understanding of other aspects of emotional function? Here we consider ways in which work on repression might inform the study of, on one hand, emotional memory, and on the other, the emotional numbing seen in patients with chronic persistent depersonalization symptoms.

We commend Erdelyi on a lively and authoritative survey of the theoretical and experimental literature surrounding repression. His central thesis - that a single model of repression can encompass a range of effects that may appear contradictory when viewed through the prism of earlier models - is persuasively argued, and we will not seek to raise objections to it here. Rather we wish to explore two questions touched on by Erdelyi at different stages in his argument. The first concerns the interactions of emotion and memory - specifically, the effects of emotionality on memory for central and peripheral information (see target article, sect. 2). The second concerns the relevance of theories and studies of repression for the understanding of wider issues of normal and abnormal emotional function. In particular, we discuss abnormalities of emotional experience that characteristically occur in depersonalization disorder.

With regard to the effect of emotion on memory, Erdelyi states that "there is widespread consensus that emotionality enhances memory for central elements of stimuli but degrades memory for peripheral items" (sect. 3.2.1.1, para. 2). In fact, although the experimental psychology literature has invariably supported the first part of this formula (enhanced central item memory), there is a long history of conflicting results as regards the second part, with some studies finding that memory for peripheral, or contextual, material is also enhanced (for a review, see Burke et al. 1992). The key issue here is likely to be the degree of emotional arousal - as Erdelyi outlines, when central information is highly arousing, as in the case of "weapon focus," it is probable that attentional resources are captured to the point where processing of peripheral information suffers. However, if the central information is less overwhelmingly arousing, and thus less attention-capturing, the emotional enhancement of memory may involve contextual information, too. This is important, as it suggests that contextual information is handled differently depending on the degree of emotional arousal, and implies that different neural circuits are involved in the processing of central and peripheral material. In recent years a number of neuroimaging studies have attempted to address these issues at a neurocognitive level (see e.g., Maratos et al. 2001; Medford et al. 2005a; Smith et al. 2004) - although emphases and methods of these studies vary, it is notable that such studies have mostly examined the functional neuroanatomy of contextual recall that is either enhanced, or, alternatively, not significantly affected, by emotional context. In our laboratory, we have been able to repeatedly demonstrate enhanced contextual recall using a verbal memory paradigm, the effect being robust across various experimental conditions (Brierley et al., in press), while current neuroimaging data provide converging evidence for a specific role for left parahippocampal gyrus in the recall of contextual material encoded in an emotional setting (Medford et al. 2005a).

All this begs the question - what of repression? It seems likely that demonstration of a possible repression effect would require more arousing stimuli, such as that used in the Erdelyi and Appelbaum experiment (described in the target article, sect. 3.2.1.1) - however, as Erdelyi comments, diminished processing of peripheral material cannot be automatically presumed due to repression. Nevertheless, such a study would readily lend itself to adaptation for a functional neuroimaging setting, and could help provide a more complete picture of the functional neuroanatomy of memory-emotion interactions.

Our second point concerns the possibility of applying insights from the study of repression to clinical groups. We have a particular interest in depersonalization disorder (DPD), an unusual psychiatric condition that has been studied relatively little until recently (for an overview of the condition, see Baker et al. 2003; Medford et al. 2005b; and for two recent large case series, see Simeon et al. 2003). In this condition, patients report a pervasive, disturbing sense of unreality in their sense of themselves (depersonalization) and their surroundings (derealization). Very often this is accompanied by a loss or diminution of subjective emotional experience, particularly in emotional responses to other people or external events. A recent analysis of patient responses to a symptom rating scale identified 
emotional disturbance as a key symptom domain of the condition (Sierra et al. 2005). And an fMRI study in which patients with DPD viewed aversive images showed that patients lacked the normal neural response to emotional stimuli, while activation in right-sided ventral prefrontal cortex was seen in DPD patients viewing aversive images, but not in controls or patients with obsessive-compulsive disorder (Phillips et al. 2001). Interestingly, this same brain region has been identified as a key area in two studies in which normal controls were asked to suppress their emotional responses to aversive images (Ochsner et al. 2004; Ohira et al. 2006). If similar neural circuitry is involved in the dampening of emotional responses, irrespective of whether this process is voluntary or not, this provides an intriguing correlate to Erdelyi's suggestion that repression can be viewed as a single entity, regardless of whether it is conscious or unconscious. It is debatable whether a general reduction in emotional responsivity would fall under the heading of "repression," as there may be no specific memory or other material that is repressed, but it could nevertheless be argued that a general lessening of emotional experience represents a form of repression: a repression of experience rather than of knowledge. In any event, whatever terminology one chooses, it seems likely that the kind of "de-affectualization" seen in DPD has some connection with repression as discussed by Erdelyi, and might usefully be illuminated by the kind of theoretical analysis and experimental approaches outlined in his excellent article.

\section{The United States of Repression}

\author{
Sadia Najmi and Daniel M. Wegner \\ Department of Psychology, Harvard University, Cambridge, MA 02138. \\ najmi@fas.harvard.edu wegner@wjh.harvard.edu \\ http://www.wjh.harvard.edu/ wegner/
}

\begin{abstract}
Erdelyi's account of thought suppression, which he equates with the Freudian construct of repression, is that it is mostly successful, and that it undermines memory for the suppressed material. Erdelyi has neglected to consider evidence from two decades of research on suppression which renders both these claims invalid. Contrary to Erdelyi's thesis, suppression often enhances the accessibility of unwanted material.
\end{abstract}

As Freud's major interpreter to experimental psychology for the past several decades, Matthew Erdelyi has contributed a wealth of research on repression. He has certainly earned the right to suggest the shape of future theory and research on this topic, and in the target article, he makes a strong suggestion. His message in this unified theory of repression is that Freud often used the terms repression and suppression interchangeably (in that repression may be both conscious and unconscious), and that everyone is getting it all wrong by perpetuating a false distinction between these processes. Erdelyi may be right about Freud, but he is not right about the human mind. There are good reasons to retain the distinction, not the least of which is the well-established finding Erdelyi somehow fails to recognize - that suppression can often have ironic effects, enhancing the recurrence of unwanted thoughts rather than effectively eradicating them.

First off, trying to unite the terms suppression and repression makes little sense if there is a clear conceptual distinction between them (no matter what it was that Freud intended). The fact that these terms have now survived for years in the psychological lexicon suggests that they do capture a valuable distinction. After all, some mental processes occur unbidden, whereas others occur voluntarily; why shouldn't we celebrate this truth in the case of the process of not thinking? This partition was highlighted initially by studies that asked people to try consciously to suppress thoughts, and it is in the understanding and appreciation of this research that Erdelyi's unified theory fails most seriously.

Citing Wegner (1989), Erdelyi mentions the "white bear" findings - that people who try not to think about a white bear and are later allowed to think about it show a relative rebound of the thought. He intimates that the effect fails to replicate - by citing an unpublished dissertation by Gildston (1998) and a study by Anderson and Green (2001) that did not even test for the rebound effect. This review is so superficial as to be seriously misleading. No mention is made of the scores of studies showing the return of suppressed thoughts, both in post-suppression rebound and also during suppression under conditions of mental load (see reviews by Abramowitz et al. 2001; Beevers et al. 1999; Rassin 2005; Wegner 1989; Wenzlaff \& Wegner 2000).

Erdelyi also neglects to mention the critical factor protecting mental control from its ironic effects - the availability of mental capacity (Wegner 1994). When capacity is compromised by cognitive load, the control falls below a baseline level and produces the opposite of the intended effect. Thus, a thought one is trying to suppress becomes more accessible under mental load. Moreover, this is not unique to thoughts, but in fact is the same process at work when people are burdened by competing cognitive demands as they try to relax (e.g., Wegner et al. 1997), concentrate (e.g., Wegner et al. 1993), sleep (e.g., Ansfield et al. 1996), avoid being prejudiced (e.g., Macrae et al. 1994), or ignore pain (e.g., Cioffi \& Holloway 1993).

Erdelyi approvingly cites Freud's theory of the counter-will, apparently without realizing that it captures exactly these ironic effects. As Wegner (1994) notes in detail, the finding that unwanted thoughts surface when a person is under cognitive load follows nicely from Freud's (1892-1893/1966) description of counter-will: "The antithetic idea gains the upper hand as a result of general exhaustion" (p. 125). Whereas Freud goes on to attribute the depletion of mental capacity to sexual and aggressive conflicts, evidence from suppression research reveals that the mechanisms are less exotic (e.g., time pressures, stress, concurrent tasks, etc.).

Since suppressed thoughts return with relentless predictability, it may be tempting to attribute special psychological significance to them, perhaps even to see them, as Freud did, as the content of the deeper, disquieted self. However, what two decades of research have shown is that thoughts return relentlessly even when people are instructed to try not to think about the most mundane things such as white bears (Wegner et al. 1987), houses (Wegner \& Erber 1992), rabbits (Clark et al. 1991), and vehicles (Lavy \& van den Hout 1990).

Evidence for whether emotionally relevant thoughts are easier to suppress has been found in some studies (Kelly \& Kahn 1994; Wegner \& Gold 1995) but not in others (McNally \& Ricciardi 1996; Salkovskis \& Campbell 1994), and hence the research is inconclusive on this issue. However, in studies with clinical populations, evidence weighs heavily toward impaired suppression of emotionally relevant material. This directly contradicts Erdelyi's thesis. For example, Shipherd and Beck (1999) showed the inability to suppress rape-related thoughts in post-traumatic stress disorder (PTSD) patients, Harvey and Bryant (1998) showed the same effect for accident-related thoughts in survivors of motor vehicle accidents with acute stress disorder, and Conway et al. (1991) showed impaired suppression of negative thoughts in dysphoric individuals. Thus, Erdelyi's claim that suppression is mostly successful has not held up to empirical scrutiny.

One implication of Erdelyi's account of suppression is that it impairs memory for the suppressed material. Again, this claim has not been supported empirically. Several studies have shown instead that when suppression is relinquished or interrupted, instructions to suppress can lead to enhanced recall of the suppressed information. Because suppression enhances the accessibility of the target thought (e.g., Wegner \& Erber 1992), if anything, the enhanced attention would be expected to improve memory for the information. Indeed, some studies 
have found this to be the case. For example, the suppression of stereotype-congruent memories leads to enhanced recall and recognition of the material when suppression is discontinued (Macrae et al. 1997; Sherman et al. 1997).

A unified theory of repression may eventually be developed, but that theory cannot simply hold that all forms of not thinking are the same. Conscious suppression of thoughts often has the ironic effect of accentuating those thoughts, and a full theory of repression must both acknowledge and explain the occurrence of such effects.

\section{Social incoherence and the narrative construction of memory}

\section{Judith Pintar ${ }^{\mathrm{a}}$ and Steven Jay Lynn ${ }^{\mathrm{b}}$}

${ }^{a}$ Department of Sociology, University of Illinois at Urbana-Champaign, Urbana, IL 61820; ' Department of Psychology, State University of New York at Binghamton, Binghamton, NY 13902.

jpintar@uiuc.edu slynn@binghamton.edu

Abstract: By shifting the focus of analysis from forgetting and remembering to interpreting and making-meaning, Erdelyi allows theoretical consideration of repression to move beyond the heuristic assumption that personal memory is necessarily private memory. In this commentary, repression is considered to be a collective process in which memories are shaped by the need for coherence between individual and social narratives.

Erdelyi's integrative theory pivots on the idea that repression represents a broad class of consciousness-lowering processes. If repression is merely an umbrella term for the tandem operation of cognitive avoidance and transformations of target material, we find little to quibble with in this regard. Nor do we contend with Erdelyi's view - now widely accepted in the scientific community - that memory construction is a complicated, active, and meaningmaking process. Although we appreciate the breadth of the integrative theory, which encompasses developments in evolutionary theory, neuroscience, and experimental research in memory, Erdelyi's focus is still too narrow. Remembering and forgetting are also collective processes, and as such are subject to specific narrative pressures. On one hand, there are the multiple ways in which attention is directed to salient social events, including reinforcement ("Yes, that's what happened"), suggestion ("Do you remember when that happened?"), and rehearsal ("Tell everyone what happened"). On the other hand, there are narrative processes of directed inattention ("Don't tell, don't think about it, it didn't happen"), which occur within relational contexts marked by varying degrees of coercion and constraint.

Throughout Erdelyi's exposition, the question hovers regarding what the construct of "repression" adds to our understanding of human memory beyond ordinary forgetting. Yet, by shifting the focus of analysis from forgetting and remembering to interpreting and making-meaning, Erdelyi allows theoretical consideration of repression to move beyond the heuristic assumption that personal memory is necessarily private memory. If memory is understood to reside in the relational space between people who share direct and indirect experiences, then its dynamics take on considerable depth. We remember and forget not only within the parameters given to us by our social worlds, but as part of the mutual creation of the narratives that give form to our social worlds.

In broad sweep, this conceptualization of memory is not new. Sociologist Maurice Halbwachs (c.1877-1944) and psychologist Charles Blondel (c.1876-1939) argued that personal memories cannot be understood in isolation from the social frameworks that give them meaning. According to Halbwachs (1951/1992), the conceptual narratives that arise within a particular cultural or social group are internalized by individuals within that group, who must construct and reconstruct their autobiographical memories within the frames they know. He notes, however, that the frameworks individuals depend upon to remember their pasts are also constructions, themselves subject to a variety of pressures. Social memory is not necessarily more accurate than personal memory, because social groups frequently distort the past in the act of reconstructing it. Rather than ensuring accuracy, social frameworks as a mechanism promote consistency in memory between individuals and social groups. Blondel, whose research and writing were informed by clinical practice, was intrigued by the in consistencies. The most pathological conditions described by Blondel (1914) were suffered by individuals whose autobiographical selves were dramatically disconnected from collectively understood frames of meaning. He theorized that his patients who were unable to retrieve certain memories had never identified an appropriate social framework within which to comprehend them (see Wallon 1951/1984).

In recent decades, research interest in the social and cultural aspects of autobiographical memory, particularly as it relates to traumatic experience, has increased (Fivush \& Haden 2003; Lynn \& Pintar 1997; Lynn et al. 1997; McNally 2003). In our analysis of the dynamics of revictimization (Lynn et al. 2003), we introduced the term "social incoherence" to refer to intolerable incongruities between personal and social narratives. A child who is abused by her father, for example, finds her experience to be incoherent within the social contexts that she knows. Her father's actions contradict cultural narratives of what fathers are supposed to do and to be, and his interpretations likely will be irreconcilable with her experience as well; he may tell her that she asked for the abuse or that it didn't even happen at all. When a child inhibits her memories of specific events and enhances other aspects of her experience, she may be attempting to bring her personal narrative in line with the social narratives. Repression, in this view, is a method of reducing the distress of social incoherence.

The concept of social coherence recalls some features of Festinger's (1957) cognitive dissonance, and Bateson's doublebind (1972), though our focus is on incompatibilities between personal experiences and collective frameworks available for comprehending those experiences. Correspondingly, a social narrative approach predicts that traumatic experiences will be followed by less memory distortion and better outcomes, generally, for those who have access to social frameworks which help them narrate their experiences coherently. Research on maltreated children demonstrating that the presence of supportive social relationships moderates risk of depression provides suggestive evidence for this hypothesis (Kaufman et al. 2004).

A growing base of research supports the view that traumatic events can compromise the coherence of memory and narrative production. To be more specific: (1) rape narratives are more ambiguous than narratives related to non-rape events (Tromp et al. 1995); (2) the level of articulation of trauma narratives is inversely related to chronic post-traumatic stress disorder (PTSD) (Amir et al. 1998); and (3) reductions in traumarelated anxiety during therapy for PTSD are associated with a decrease in the fragmentation of rape narratives (Foa et al. 1995a). Moreover, emotional processing leads to the creation of organized, unfragmented, and more articulated narratives related to traumatic events (Amir et al. 1998; Foa \& Riggs 1993). Social coherence, however, cannot be achieved solely through the cognitive task of modifying personal narratives, in cases when it is the social world and its narratives that are inaccurate and require alteration (Pintar 2000).

Erdelyi recognizes that there are multiple interpretive contexts surrounding any event, and that individuals will weight these differently. We see weighting as an intrinsically social and relational process in which individuals are subject to direct and indirect pressures of reinforcement and constraint. Erdelyi is correct that we inhibit and enhance experiences to make sense of our reality. We would add that we are often compelled to make that reality coherent in relationship to larger social realities. 


\section{From repression and attention to culture and automaticity}

\section{Amir Raz ${ }^{\mathrm{a}}$ and Horacio Fabrega, $\mathrm{Jr}^{\mathrm{b}}$ \\ ${ }^{a}$ Vancouver Coastal Health Research Institute and University of British Columbia, VGH Research Pavilion, Vancouver, British Columbia, V6H 3Z6, Canada; ${ }^{b}$ University of Pittsburgh, Department of Psychiatry, Pittsburgh, PA 15216}

DrAmirRaz@gmail.com

\section{hfabregajr@adelphia.net}

\begin{abstract}
Erdelyi grants "repression" emotional and cognitive qualities that can modulate consciousness and probably overlap with what is typically attributed to "attention." Such a broad appellation of repression explains virtually all behavior and lacks specificity. Repression and attention elucidate behavior in different clinical, cognitive, and cultural contexts Refining these influences, we identify a few lacunae in Erdelyi's account.
\end{abstract}

Erdelyi competently threads through the clinical and experimental literature, expanding and redefining "repression" so as to uphold Freud and question post-Freudian psychoanalysis. Repression is placed on and straddles the conscious-nonconscious continuum; all "defense mechanisms" are subsumed as "elaborative" mechanisms. Erdelyi's repression presupposes several mental faculties (e.g., self awareness, reasoning, language, and memory) and engulfs too many behaviors; that is, it lacks specificity.

It is unclear why repression should be defined as consciousness-lowering; even Erdelyi's own analysis construes it as consciousness-feeding or consciousness-regulating. Similar to the operationalization of attention, repression implies that significant information (e.g., stimuli and memories) is selected and deployed to reach a goal in the context of motivational, emotional imperatives. Indeed, both attention - especially when construed as an organ system (Posner \& Fan, in press) - and repression subserve overlapping functions. And yet, they appear to differ in a specific context: Whereas repression is an ulterior psychopathological notion, researchers have unraveled a great deal about theories, mechanisms, and typologies of attention in healthy as well as pathological populations (Raz \& Buhle 2006).

Attention is a strong regulator of cognition, emotion, and action. In the present context, we speak of executive attention, which goes by many names including supervisory, selective, conflict resolution, and focused attention. This form of attention relates to self-regulation (i.e., the ability to manipulate one's own emotions, thoughts, or actions upon direction from the self or another person), emotional-regulation (i.e., the reduction, increase, or sustaining of an emotional response such as fear, anger, or pleasure based upon the actions of the self or others), ${ }^{1}$ effortful control (i.e., the ability to inhibit, activate, or sustain a response, which includes the capacity to inhibit a dominant response in order to perform a subdominant response), and inhibitory control (i.e., the reduction in the probability, speed, or vigor of the normal response to a stimulus based upon instruction from the self or others). Both repression and attention help explain similar cognitive phenomena. However, one caveat of Erdelyi's model is that it fails to address cognitive influences such as automaticity and culture. A unified theory of repression and attention should help explain not only psychopathology (e.g., defense against unconscious) but also phenomena such as trance, "dissociated" behavior, hypnosis, and other culturebound syndromes of psychiatric interest. Indeed, clinical psychiatry, medical anthropology, and the social history of medicine document that such phenomena represent human universals (Fabrega 1975; 1997). These accounts describe observable syndromes of behavior that are culturally constructed and appear standardized, if not largely automated, yet subject to different externally viewed interpretation (Simons \& Hughes 1985).

Cognitive psychologists generally agree that mental processes come in two forms, automatic and controlled, and that automatic processes are either innately automatic, or become automatized through extensive practice (Shiffrin \& Schneider 1977; Spelke et al. 1976). Erdelyi's variant of repression seems automatic in this sense. However, some individuals (e.g., highly suggestible people) can "un-ring the bell" and regain control over something that's been automatized (Raz et al. 2006). The literature offers little or no discussion of this issue, leaving the question unanswered and mostly unasked, although certain meditative disciplines describe achieving the "de-automatization" of thought and at least a few reports support such accounts (Alexander et al. 1989; Dillbeck 1982; Wenk-Sormaz 2005). Erdelyi provides but a glimpse into the relationship of repression to automaticity and stops short of contextualizing how gaining control over an automatic process may relate to repression. Such treatment is sorely missing from his account.

Culture is expectation-driven behavior, and products of "enculturation" reside in brain networks that influence processes such as awareness, memory, and action. As a pool of information, culture allows individuals to shape behavior and habit patterns and informs as to what is acceptable and normative. As it evolved, it set the stage on which individuals could jointly work out their circumstances in a personal and group-centered way (Fabrega 2002). Culture influences how attention and repression function in a context of competing impulses, values, and goals toward adaptive behavior (Raz et al. 2005). Culturally encoded information influences much of what psychiatric disorders produce in observable behavior (Fabrega 1975). Similar to individual and genetic variations (Fan et al. 2003; Mayr et al. 2005), culture is also wired into the brain, and the neural manifestations of disparate cultures likely correlate with different neural patterns. For example, bicultural individuals can assume different roles as a function of environmental cues, and bilingual persons can report feeling like a different person depending on the language they use. Thus, personality can change as a function of language and culture, including historical periods and mentalities, suggesting that personality is perhaps more malleable, and culture more influential, than is commonly held. Although powerful cultural signals can be offset by awareness and experience, the contextual nature of personality is of importance in a world that is increasingly multicultural and multilingual.

In the context of conflict resolution and conflict monitoring (Bush et al. 2000), one would expect attention, repression, or another suitable behavior-regulating mechanism to explain processing items of information over brief time. However, it should also help explain the larger whole producing extended syndromes of symbolically meaningful and observable behavior that include regulation and coordination of mental material and deployment of motor components (Garro 2001).

In unified context, repression, attention, and culture are useful to the extent that their mechanisms and effects are identifiable and measurable. Attention is well within this scope; repression and culture less so. However, the concept of repression seems to serve a heuristic purpose because researchers can use it to make predictions concerning new studies. Although it is unclear whether Erdelyi's theory is falsifiable in the Popperian sense, a brief glance into the history of science shows that multiple theories were not falsifiable initially, not because they were not sufficiently well operationalized in terms of measurable variables (e.g., in Freudian theories), but because they were not fully developed. We hope that Erdelyi's account will be extended and revised to permit more testable predictions as additional research is conducted using new methodologies.

NOTE

1. Emotion regulation can be a form of self-regulation but it could also be induced by the actions of others.

\section{Towards a post-Freudian theory of repression: Reflections on the role of inhibitory functions}

\author{
Ralph E. Schmidt and Martial Van der Linden \\ Department of Psychology, University of Geneva, Geneva, 1205, Switzerland. \\ Ralph.Schmidt@pse.unige.ch Martial.Vanderlinden@pse.unige.ch
}


Abstract: Although Freud's merits may be readily acknowledged in the year of his 150th birthday, recent findings on repression-related phenomena cannot be accommodated by his classic conception, on which Erdelyi's theory is built. This point is illustrated by discussing the role of inhibitory processes. The unified theory of repression should be elaborated to generate falsifiable predictions on the reported phenomena.

Although we welcome Erdelyi's endeavor to integrate insights from different clinical and laboratory traditions into a unified theory of repression, we consider that the framework he proposes should be elaborated in ways that lead beyond Freud's classic conception. Building on Freud's distinction between repression in the narrow sense and repression in the widest sense (Freud 1937/1964), Erdelyi proposes that repression is divided into two subclasses, inhibitory (or simple) repression and elaborative repression. He affirms that these two subclasses of "consciousness-lowering processes" are "extensively buttressed ... by the experimental literature.” Inhibition consists on the mental level in "cognitive avoidance (not-thinking)" or "subtract[ion of] attentional allocation" that results in "degrading the "signal" (sect. 3.1). For the underlying mechanisms, a parallel is drawn between inhibition and the functioning of inhibitory circuits in the brain.

Appealing as it may appear with its neural nimbus, the concept of inhibition is far from being unanimously accepted in cognitive psychology: in fact, it has been seriously challenged from different sides. The most fundamental challenge has come from authors who have gone so far as to question the "right of existence" of this concept; they propose that experimental effects generally attributed to inhibition are amenable to alternative explanations. MacLeod et al. (2003), for example, offer such "inhibition-free explanations" for results typically obtained with the directed forgetting (DF) and the retrieval-induced forgetting (RIF) paradigms. MacLeod et al. mention, among the candidate mechanisms that may replace inhibition, selective rehearsal (for DF) and retrieval strategy disruption (for RIF). Erdelyi invokes the results typically obtained with these paradigms as evidence in support of the notion of inhibitory repression; at the same time, he surmises that mechanisms such as selective rehearsal and selective search might also be tapped by these tasks. In this respect, his theory must be qualified as underdetermined: It specifies neither the conditions in which the different mechanisms are called upon, nor their possible interaction, nor the reasons that an inhibition-involving account is superior to an inhibition-free account.

In a similar vein, the literature on thought suppression (for a review, see Wenzlaff \& Wegner 2000) that Erdelyi briefly mentions suggests that mental control may be modeled without recourse to the concept of inhibition. The leading theoretical account in this field, Wegner's ironic process theory (Wegner 1994), posits an interaction between an effortful intentional operating process that seeks distracters (thoughts other than the tobe-suppressed target) and a less effortful ironic monitoring process that watches for intrusions of the target in order to alert the first process of the need to renew distractions. The postsuppression rebound of the target is explained by the fact that when the operating process is voluntarily relinquished or disrupted by cognitive demands (or resource depletion as during sleep; Schmidt \& Gendolla 2006; Wegner et al. 2004), the monitoring process continues its vigilance for unwanted thoughts, thereby enhancing their activation. By this view, suppression implies a mechanism of selective attention, but not necessarily one of inhibition (for a similar position, see Engle 2000).

Another challenge for inhibition has come from studies showing that this concept rests on a fragile empirical foundation. For example, Salthouse et al. (2006) have recently examined six tasks that are often interpreted in terms of inhibition-related memory control; among them were, again, DF and RIF tasks. Analysis of the relations between the variables derived from these tasks did not yield any significant sign of convergent validity for one or more memory-control constructs. Even though this finding may be ascribed to the poor reliability of the memorycontrol measures used, it constitutes a serious challenge to any inhibition-related repression account. Investigations of the neural substrates of inhibition have not produced unequivocal evidence for this mechanism either. For example, Collette et al. (2005) conducted a study using positron emission tomography to explore the cerebral areas associated with three executive functions: updating, shifting, and inhibition. Although some regional activation patterns were common to all three functions, only a weak inhibition-specific activation was found in the right inferior frontal region. This finding may again fuel doubts as to the validity of the inhibition construct.

Critical comments about the concept of inhibition have also been made by authors who generally adhere to it; they suggest that inhibition should be conceived of as a multidimensional construct rather than as a unitary one. Friedman and Miyake (2004), for example, examined the relations between three inhibition-related functions. They found that prepotent response inhibition and resistance to distracter interference were closely related and that both were unrelated to resistance to proactive interference. In a structural equation model, these investigators combined prepotent response inhibition and resistance to distracter interference into a single latent variable and observed that it was related to everyday cognitive failures; unwanted intrusive thoughts, on the other hand, were related to resistance to proactive interference. This result highlights the interest of establishing a taxonomy of inhibition-related functions - a theoretical refinement that lacks in the unified theory of repression. Erdelyi conjectures that repression "knocks out declarative (conscious) memories" and may affect nondeclarative representations (e.g., procedural ones) in a different way, but he does not take the step of distinguishing two or more different inhibitory functions. Whether unwanted intrusive thoughts or everyday cognitive failures (slips according to the Freudian terminology) are concerned, the inhibitory mechanism acting upon them is thus thought to be the same.

In light of the reported findings and theoretical accounts, we suggest that the unified theory of repression should be elaborated to be more specific about the implication of inhibitory processes. First, it should be made clear whether the term inhibition refers to a mental operation ("not-thinking of something") or to a cognitive mechanism that is supposed to explain behavior; this distinction is not always neatly drawn in Erdelyi's article. Second, if a cognitive mechanism of inhibition is postulated, arguments for the superiority of such an account over an inhibition-free account of repression should be provided. And third, the concept of cognitive inhibition should be broken down in terms of separable functions. It is our belief that these suggestions could lead to the generation of novel, testable, and thereby falsifiable hypotheses about repression.

\section{Repression and dreaming: An open empirical question}

\section{Michael Schredl \\ Sleep laboratory, Central Institute of Mental Health, J5, 68159 Mannheim, Germany. \\ Schredl@zi-mannheim.de www.dreamresearch.de}

Abstract: From the perspective of modern dream research, Freud's hypotheses regarding repression and dreaming are difficult to evaluate. Several studies indicate that it is possible to study these topics empirically, but it needs a lot more empirical evidence, at least in the area of dream research, before arriving at a unified theory of repression.

From the perspective of modern dream research, Freud's hypotheses regarding repression and dreaming are difficult to 
evaluate. Since Erdelyi makes several references to Freud's dream theory (e.g., dream work as example of elaborate repression processes), it seems important to discuss this topic.

Freud (1900/1987) hypothesized that dreams with unsuccessfully concealed wishes are repressed (not remembered) as a whole. This hypothesis, however, cannot be tested empirically since the repressed dream is not available and cannot be compared to recalled dreams. Indirect approaches linking trait repression to dream recall frequency have not been successful. most studies did not find a significant relationship (for an overview, see Schredl \& Montasser 1996-97).

Similarly, the processes of rationalization, symbolization, projection, reversal, and displacement, as a set of techniques by which the latent dream content is transformed into the manifest content, cannot be tested in a direct way, because the latent content is a theoretical construct and, therefore, not observable. Freud's clinical practice (i.e., starting from the manifest dream and working through to the hypothesized latent meaning) does not suffice modern scientific standards. It is important to complement this approach with empirical studies, an issue which is stressed by Erdelyi several times. Most dream content research has been carried out under the premise of the continuity hypothesis of dreaming (cf. Schredl 2003), which - in its general form - simply states that waking life is reflected in dreams. Factors that might be important in explaining the incorporation of waking-life events (or thoughts, emotions, concerns) into dreams, such as emotional involvement, have also been proposed (Schredl 2003). Schredl and Hofmann (2003), for example, reported that the amount of time driving a car is positively related to the frequency of driving dreams. Another finding is that depressive mood is correlated with negatively toned dreams and dream themes of death and aggression (Schredl \& Engelhardt 2001). These straightforward effects of waking life on subsequent dreams have also been acknowledged by Freud (1900/1987) as day residues. Although these kinds of paradigms are easily put into research practice and, hence, empirical evidence is accumulating, more sophisticated approaches to test Freudian notions have rarely been carried out. Cartwright et al. (1969) showed an erotic movie to 10 young men and found more male sex symbols (gun, knife, tool, golf club) and female sex symbols (box, tunnel, corridor) in the dreams after the film (but not more direct incorporations of the film), a finding that was interpreted as occurrence of symbolization within these dreams.

Wegner et al. (2004) applied a simple suppression strategy ("Do not think about this person") prior to bedtime and found a higher incorporation rate of the target person $(34.1 \%)$ than after the expression condition (thinking about the person the same amount of time prior to bedtime; 28.2\%). Although this procedure might not be called complete repression (the target person occurred on average about three times within the 5-minute stream-of-consciousness report), the authors clearly demonstrated that hypotheses concerning repression and dreaming can be studied empirically. It might be possible to study whether repressed thoughts in everyday life are also often incorporated in dreams.

Erdelyi mentions two studies (Pötzl 1917; Fisher 1956) regarding the effects of subliminal presented stimuli on dreams. Although there are studies clearly demonstrating effects of subliminal perception on the organism (e.g., fear reactions in patients with spider phobia) even if they were not able to recognize the pictures presented for a very short time (Öhman \& Soares 1994), the studies mentioned by Erdelyi have several methodological flaws (no control condition, a vague interpretation of coincidences between dream drawings and original picture, tachistoscopic presentation [ignoring the iconic memory]). A more sophisticated study (Schredl et al. 1999) using masking procedures for presentation the target pictures, a control condition, and precisely defined rating scales for the analysis of the pictures, also found an effect of subliminal stimuli on subsequent dreams (increased number of objects and concepts represented in the pictures). Because of the small sample size, further corroboration of these findings is necessary.

To summarize, Erdelyi's undertaking to present clear definitions of repression and repression processes is a fruitful one for future research; but it needs a lot more empirical evidence, at least in the area of dream research, before arriving at a unified theory of repression.

\section{The mnemic neglect model: Experimental demonstrations of inhibitory repression in normal adults}

\author{
Constantine Sedikides ${ }^{a}$ and Jeffrey D. Green ${ }^{b}$ \\ ${ }^{a}$ School of Psychology, University of Southampton, Southampton SO17 1BJ, \\ England, United Kingdom; ${ }^{\mathrm{b}}$ Department of Psychology, Virginia \\ Commonwealth University, Richmond, VA 23284-2018. \\ cs2@soton.ac.uk \\ http://www.soton.ac.uk/ crsi/constantineprofile \\ jdgreen@vcu.edu http://www.has.vcu.edu/psy/people/green.html
}

Abstract: Normal adults recall poorly social feedback that refers to them, is negative, and pertains to core self-aspects. This phenomenon, dubbed the mnemic neglect effect, is equivalent to inhibitory repression. It is instigated under conditions of high self-threat, it implicates notthinking during encoding, and it involves memories that are recoverable with such techniques as recognition accuracy.

In a laboratory program of research, Sedikides and Green (2000) have demonstrated what is in essence inhibitory repression in normal adults. Our starting point was the assumption that people receive mixed (i.e., positive and negative) feedback in their daily lives. For example, an employer may praise them for the completion of a project but point out that the outcome could have been more successful. A friend may remark that she values their dependability but not their fashion sense. A partner may exalt them as a lover but lament their hygiene habits. How do people process mixed feedback? Does it matter if the same feedback refers to the self as opposed to another person? Stated differently, do people process and remember self-referent feedback differently than other-referent feedback? More interestingly, how do people defend the self (compared to another person) from threatening information?

We introduced the mnemic neglect model (Sedikides et al. 2004) to address these issues. The model endorses the notion that people are motivated to defend, maintain, or increase the positivity of the self-concept (Sedikides \& Gregg 2003; Sedikides \& Strube 1997). In addition, the model draws a distinction between negative versus positive feedback, central (i.e., pertaining to relatively high certainty, descriptiveness, and importance self-aspects) versus peripheral (i.e., pertaining to relatively low certainty, descriptiveness, and importance self-aspects) feedback, and self- versus other-referent feedback (Sedikides 1993; 1995). Negative, central, and self-referent feedback is selfthreatening. The other categories of feedback are either low self-threat (i.e., positive central self-referent, positive central other-referent, negative central other-referent) or tangential to the self (i.e., positive peripheral self-referent, negative peripheral self-referent, positive peripheral other-referent, negative peripheral other-referent).

The model posits that people neglect disproportionately the processing of self-threatening feedback. Such feedback (e.g., "You would purposely hurt someone to benefit yourself") is inconsistent with one's self-view (e.g., kind), and one cannot imagine behaving in such a rude manner. Thus, self-threatening feedback is processed shallowly. Little, if any, elaboration (i.e., association with similar behaviors) occurs, resulting in a decreased number of retrieval routes and, hence, poor recall. In contrast, low self-threat feedback (e.g., "You would offer to 
care for a neighbor's child when the baby-sitter couldn't come") is consistent with one's self-view, and one can easily imagine behaving in such a kind manner. Thus, low self-threat feedback is processed deeply. A substantial degree of elaboration occurs, resulting in an increased number of retrieval routes and, hence, better recall.

In our typical experiment, participants are presented with 32 behaviors that are either positive or negative and exemplify either central (i.e., trustworthy, kind) or peripheral (i.e., modest, uncomplaining) self-aspects. Half of the participants are instructed to think of the description as based on actual knowledge that familiar others have of them, whereas the other half are told that the description refers to another person named Chris. The typical finding (Sedikides \& Green 2000 Experiment 2) is that participants remember relatively poorly self-threatening (i.e., negative central self-referent) behaviors. This finding has been replicated in a situation of relatively high mundane realism, in which the feedback was based on an ostensibly valid personality test (Sedikides \& Green 2000, Experiment 1 ). The finding has been labeled the mnemic neglect effect and is taken as evidence of self-defense. This effect is conceptually equivalent to inhibitory repression, which, according to Erdelyi, involves "cognitive avoidance (not-thinking)" of threatening material and "leads to loss of accessible memory" (sect. 1, para. 2).

Subsequent experiments have established that self-threat drives the mnemic neglect effect. Sedikides and Green (2004) have shown that feedback negativity (indicating self-threat), rather than feedback inconsistency, is responsible for mnemic neglect. Green and Sedikides (2004) have shown that the effect is obtained when the behaviors are diagnostic (indicating selfthreat) rather than non-diagnostic of the underlying trait. Finally, Green et al. (2005) have shown that the effect is present when the negative feedback is targeted toward unmodifiable self-aspects (indicating self-threat) rather than modifiable self-aspects.

Inhibitory repression, according to Erdelyi, involves cognitive avoidance, or not-thinking. Does the mnemic neglect effect also involve not-thinking? Sedikides and Green (2000, Experiment 3) manipulated behavior reading time by presenting behaviors one at a time on computer screens: Some participants were given ample time (i.e., 8 seconds per behavior); others, limited time (i.e., 2 seconds per behavior). The mnemic neglect effect was replicated in the former condition but was absent in the latter. When in a not-thinking (i.e., limited time) condition, participants neglect all behaviors, not just self-threatening ones. This pattern is consistent with Erdelyi's conceptualization of inhibitory repression.

What happens to neglected memories? Are they lost or available and recoverable? Erdelyi argues that memories can be recovered with retrieval effort or alternative techniques. We employed such a technique, recognition accuracy (Green, Sedikides \& Gregg 2006). Participants were provided with the usual 32 behaviors and asked to recall them. Next, they were provided with a new set of similar 32 behaviors and asked to recognize them (i.e., state whether each of the 64 behaviors was previously presented). The results were informative. The mnemic neglect effect was replicated in recall but not recognition. That is, recognition accuracy did not differ as a function of feedback self-threat. It appears that self-threatening information was available in memory and accessible through the technique of recognition. This pattern is congruent with Erdelyi's theorizing.

In summary, our laboratory research on normal adults validates Erdelyi's mechanism of inhibitory repression (i.e., mnemic neglect effect) and extends it. This mechanism occurs in normal adults, in reference to the self, and in relatively highthreat situations (i.e., diagnostic feedback targeted to unmodifiable central self-aspects). Importantly, though, even what we labeled "self-threatening" feedback does not appear to be terribly self-threatening. Although in one experiment it was ostensibly based on a computer-administered personality test, in all other experiments it was based on hypothetical feedback. Also, in all cases, it referred to a mere likelihood of the person performing an undesirable behavior. We conclude that normal adults are remarkably intolerant even to the potential of self-threat: They hastily deploy the mechanism of inhibitory repression or mnemic neglect to ward off relatively innocuous signs of self-threat.

\section{Repression, suppression, and oppression (in depression)}

\section{Golan Shahar \\ Department of Behavioral Sciences, Ben-Gurion University of the Negev, Beer-Sheva, 84105, Israel. \\ shaharg@bgu.ac.il}

\begin{abstract}
Erdelyi's two key tenets - that repression may be conscious ("suppression") and that it is context-sensitive - resonate well with findings on unipolar depression. Drawing from this field, I argue that (1) "oppression," namely, pressure from significant others to refrain from attending to certain mental contents, influences individuals' repression/suppression; and that, (2) individuals actively create the very contexts that facilitate their repression/suppression.
\end{abstract}

Following Erdelyi's example of integrating clinical and research material, I would like to resurrect a previously published clinical case (Shahar 2004). Ms. J., a 55-year old woman, was referred to psychotherapy following a severe major depressive episode. An ambitious public relations agent, she always emphasized selfcontrol, achievement, and self-sufficiency over issues of interpersonal relatedness. This she did as a response to tremendous pressure exerted from members of her family of origin, who continuously conveyed to her that being weak and needy was reprehensible. She left home at the age of 15 , successfully graduated from high school and college, and developed a fulfilling professional life.

What was repressed/suppressed by Ms. J. was an array of mental contents (thoughts, feelings, wishes), pertaining to dependency needs; specifically, her needs to feel nurtured, protected, and cared for. Such repression/suppression was strikingly evident in her marital relationship. She met her husband in college and married him shortly after they had begun dating. In treatment, she described him as a kind, gentle, and emotionally stable man, yet, claimed she had never loved him and was quite puzzled as to why she remained in the relationship. It took her some therapeutic working through (cf. Freud 1914a/ 1958), to realize that her (repressed/suppressed) dependency, meeting her husband's dependability, served as the driving force of their marriage. Consistent with Erdelyi's position whereby "repression" need not be unconscious, it is plausible that Ms. J. consciously and deliberately refrained from getting in contact with her dependency. For instance, she vividly recalled her vowing "not to trust anyone but herself" (Shahar 2004, p. 372) and alluded to repeated efforts on her part to disavow needing, longing, and belonging.

The benefit of Erdelyi's theoretical postulate is twofold. First, it links research and clinical discourse on defense mechanisms with conceptualization and research on automatic - but conscious - cognitive processing. Considering, for example, Tzelgov's (1997) position that "Automatic but conscious: That is how we act most of the time," and his distinction between intentional (i.e., goal-oriented) and autonomous automatic processing (pp. 218-219), neither of which have to be unconscious, it follows that repression/suppression, particularly when used as a defense mechanism, constitutes a powerful example of intentional automatic processing. 
Second, the intentional nature of defense mechanisms highlights their interpersonal nature. The decision to attend, or not to attend, to various mental contents is very much influenced by what significant others deem appropriate or not. This is obvious in Ms. J.'s case, given that her family members explicitly conveyed to her the reprehensibility of dependency. Two theoretical and empirical contributions, both of which aim to account for the fact that women are twice as prone as men are at being diagnosed as suffering from unipolar depression, indirectly allude to the interpersonal nature of repression/suppression. Nolen-Hoeksema (1990) theorized that men are likely to respond to emotional distress by distracting behavior that short-circuits depression, whereas women are likely to engage in self-focus ruminating attention, which ultimately exacerbates their depression. As acknowledged by Nolen-Hoeksema (1990, p. 276), the origins of these different response styles are likely to be social, constituted through stereotypes (i.e., women are passive and emotional) and amalgamated in the context of family relations (women are discouraged from being active and assertive). Similarly, Jack's (1999) self-silencing theory holds that depressed women tend to "silence" (in cognitive terms - not attend to) self-aspects that are perceived to compromise close relations. This, in turn, leads to an increase in women's hopelessness about "the possibility of genuine relationships or selfexpression” (Jack 1999, p. 226), ultimately leading to depression. Both theoretical positions point to the possibility that social norms, expectations, and rules, impact individuals' attention and memory, leading to lowering of consciousness toward important mental contents, and in turn leading to self-alienation and depression. Hopefully, this succinct presentation will convince the reader that, at least in the case of unipolar depression, interpersonal and political oppression, operating at the cognitive level, is implicated in repression and suppression.

Further intriguing is Erdelyi's postulate, whereby repression/ suppression is context sensitive. Espousing $\varepsilon \neq \varepsilon \mid[\varepsilon]$, where $\varepsilon|| I||$ is the manifest content of the stimulus, $[\varepsilon|| I||]$ is the context, and $\varepsilon|[\varepsilon]||I| \mid$ is its latent content, Erdelyi proposes that defense mechanisms - particularly denial - are predicted by the person's failure to construe a stimulus (e.g., an event) in its proper context of meaning. In acknowledgment that repressed/suppressed mental contents are frequently enacted in relations (i.e., Freud's return of the repressed occurs in the interpersonal arena), I would like to argue that (1) people fail to grasp the meaning of these mental contents because they are eluded by some features of the social context, features which (2) they themselves created. Consider Ms. J. once again: her strong, albeit repressed/suppressed, dependency needs were constantly enacted in relationships where she repeatedly sought support and reassurance. Yet, she remained unaware of this because of her attentiveness to the boredom (in her marital relationships) and acrimony (in her relationships with therapists) summoned by these interpersonal exchanges. Ironically, it was she who actively contributed to this boredom (for instance, by putting an emotional rift between her husband and herself) and acrimony (e.g., by constantly provoking and challenging friends and therapists; see Shahar 2004). Ms. J.'s behavior, highly consistent with action perspectives of psychopathology (Shahar 2001; 2006), suggests that she actively contributed, if unwittingly, to the very social context that consolidated her repression/suppression of dependency needs.

To conclude, in drawing from research and clinical accounts of unipolar depression, I observe that (1) repression/suppression are influenced by social pressures exerted by significant others (i.e., "oppression"), and (2) this and other defense mechanisms are maintained by people being eluded by interpersonal circumstances they themselves created. These observations are made in an attempt to add an interpersonal touch to Erdelyi's intriguing, but largely cognitive, "unified theory of repression," so as to further appreciate the complex associations between cognition, power, and psychopathology.

\section{Resolving repression}

\author{
Steven M. Smith \\ Department of Psychology, Texas A\&M University, College Station, Texas \\ 77843-4235.
}

stevesmith@tamu.edu http://people.tamu.edu/ stevesmith/

Abstract: The feuding factions of the memory wars, that is, those concerned with the validity of recovered memories versus those concerned with false memories, are unified by Erdelyi's theory of repression. Evidence shows suppression, inhibition, and retrieval blocking can have profound yet reversible effects on a memory's accessibility, and deserve as prominent a role in the recovered memory debate as evidence of false memories. Erdelyi's theory shows that both inhibitory and elaborative processes cooperate to keep unwanted memories out of consciousness.

Repression has long been the battleground in psychology's family feud. No other issue has seen such contentious and emotional brawling among psychologists as the question of repression. Once seen as merely an academic debate, the memory wars now rage with such intensity not only because of theoretical disagreements and empirical squabbles, but because there have been very real victims of the war - victims of true physical and sexual abuse, and victims unjustly accused of abuse that did not actually occur. In his treatment of this divisive question, Erdelyi negotiates a peace plan for the feuding factions by reviewing the disparate views and contentions, integrating the relevant ideas and findings, correcting persistent misunderstandings, and synthesizing his unified theory of repression.

The essence of Erdelyi's theory unifies the warring factions of the memory wars by accepting and synthesizing the two positions. Erdelyi states that "repression is divided into two subclasses: (1) inhibitory or subtractive processes (e.g., degrading the 'signal'), and (2) elaborative or additive processes (e.g., adding 'noise' to the signal)" (sect. 3.1, para. 3). Erdelyi envisions these two subclasses, avoidance and distortion, not as adversarial processes, as has been assumed by the false memory debate, but rather as processes that cooperate in the service of defensive emotion regulation. This synthesis makes a lot of sense.

Some of the misunderstandings have occurred because of verbal labels. Examples of problematic labels include distinctions among the terms "repression," "suppression," and "inhibition." Erdelyi indicates that if we drop the bogus requirement that repression must be caused by unconscious mechanisms, these terms are essentially synonymous. There may be reason, however, to distinguish suppression from inhibition, even if both are important mechanisms that serve to keep unwanted thoughts and memories out of consciousness. Whereas Wegner has used the term "suppression" to refer to a temporary means of keeping thoughts out of mind (e.g., Wegner 1989), Anderson and his colleagues have used the term "inhibition" to mean the resultant effect on a memory following repeated suppression of a memory retrieval (e.g., Anderson et al. 1994; Anderson \& Green 2001).

Some misunderstandings in the memory wars have been caused by conceptual disagreements. Erdelyi decouples the concepts of "defense" and "repression," indicating that repression, as a mechanism, might or might not be engaged for the purpose of defensive emotion regulation. Psychologists who focus on the mechanisms of repression can study those mechanisms independently of defensive coping purposes. This understanding is important not only for investigating the mechanisms that underlie forgetting, such as suppression, inhibition, and blocking, but also for investigations that give rise to elaborative repression, such as false memories and memory attribution errors.

Some misunderstandings have persisted because of limited empirical evidence, or more often, because some types of laboratory research have not been commonly associated with the repression debate. The relevance of false memory research done in the laboratory to the repression debate has been made abundantly and compellingly clear by such investigators as Loftus, Roediger, and many others. Empirical studies of forgetting and recovered 
memories, however, have been cited only rarely in this debate. Erdelyi appropriately points to a long history of laboratory research on mechanisms of forgetting, dating back to Ebbinghaus, and including more recent research on suppression, inhibition, and blocking. These relatively simple laboratory procedures can induce strikingly potent forgetting effects (e.g., Smith et al 2003). Equally important is research on memory recovery, which includes both reminiscence (e.g., Erdelyi \& Kleinbard 1978) and cue-dependent recovery (e.g., Smith et al. 2003).

One piece of the big picture that Erdelyi has neglected concerns the mechanisms and consequences of memory recovery. Although he points out that forgetting need not be due to decay (or "oblivescence"), he implies that reminiscence is caused simply by persistent efforts to retrieve. Reminiscence can be caused by the same type of restructuring that can give rise to insight in problem solving, that is, by breaking mental sets that initially block successful retrieval. For example, Smith and Vela (1991) found incubated reminiscence effects, similar to incubation in problem solving, and their evidence indicates that the increased reminiscence found after a delay is not a result of simply attempting to retrieve more. Furthermore, Smith et al. (2003) showed that appropriate cues can trigger powerful memory recovery effects. The affective consequences of memory recovery may also be important, particularly if recovery is accompanied either by disturbing realizations, or by relief when restructuring makes it clear that stressful memories can be more rationally or innocently reinterpreted. More research is needed to investigate the emotional outcomes of memory recovery.

Erdelyi's unified theory of repression provides a cogent and compelling framework for understanding several bodies of research, as well as the history of psychotherapy. His theory is also important because of the questions that must now be investigated to expand the utility of his theory. One important gap is research on implicit memory of reversibly forgotten material; that is, the "return of the repressed" in the form of non-declarative memories. Other insufficiently researched questions include what are the limits of forgetting (e.g., how and under what circumstances emotional and distinctive experiences are forgotten), the limits of false memories (e.g., what degree of schema consistency must be maintained for false memories to occur), and the limits of recovered memories (e.g., how do repressed memories degrade in detail and accuracy over time). More research that informs us on the question of distinguishing between false and valid recovered memories is also needed. Finally, we must learn more about methods for recovering memories, including how those methods can enhance the amount and the accuracy of recovered memories, and what the affective consequences of recovery are likely to be.

Erdelyi correctly points out how researchers have continued to misunderstand and misinterpret each other, how we dissociate ideas and research that should be related, and how partisans in the memory war have imposed unjustified distinctions that have blurred our understanding of repression. His theory takes important steps in terms of resolving the subject of repression, and, hopefully, demilitarizing the memory wars.

\section{Author's Response}

\section{The return of the repressed}

\section{Matthew Hugh Erdelyi \\ Department of Psychology, Brooklyn College and the Graduate School, City University of New York, Brooklyn, NY 11210-2889. iyledre@patmedia.net}

Abstract: Repression continues to be controversial. One insight crystallized by the commentaries is that there is a serious semantic problem, partly resulting from a long silence in psychology on repression. In this response, narrow views (e.g., that repression needs always be unconscious, must yield total amnesia) are challenged. Broader conceptions of repression, both biological and social, are considered, with a special stress on repression of meanings (denial). Several issues - generilizability, falsifiability, personality factors, the interaction of repression with cognitive channel (e.g., recall vs. dreams), and false-memory as repression - are discussed.

\section{R1. Introduction}

It is perhaps not surprising that, given the controversial nature of repression in modern psychology, reactions to "The Unified Theory of Repression" (TUTOR) vary widely in the commentaries, ranging from outright rejection of the reality of repression (e.g., Bonanno; Hayne, Garry, \& Loftus[Hayne et al.]; Kihlstrom; McNally) to urgings that the ambit of repression be extended further than I ventured in my target article (e.g., Freyd; Hermans, Raes, Iberico, \& Williams [Hermans et al.]; Pintar \& Lynn; Shahar; Smith). The commentaries, as a group, are extremely helpful in revealing the issues animating the repression controversy, and I am grateful for the commentators' efforts, regardless of their stances.

Several important, partly overlapping themes emerged in the commentaries, to which I respond here. First (see sect. R2), it is increasingly clear that the controversy surrounding repression is to an important degree semantic. Extremely narrow or stringent definitions of repression (e.g., Bonanno, Crews, McNally, Hayne et al.) tend indeed to render repression nonexistent, whereas more expansive conceptualizations (which I claim are consistent with the classical literatures) render it ubiquitous and obvious.

A related issue (sect. R3) is the striking hiatus in the scientific literature on repression for a generation, which I claim (contrary to McNally) is partly responsible for the depredations of the memory-recovery therapy movement. Also, this long silence on repression contributed to the semantic derailment of the concept. Thus, I oppose some of the commentators' apparent eagerness (e.g., Bonanno, McNally) to reestablish psychology's silence on repression. Critics of repressed memory (e.g., Bonanno, Hayne et al.; Kihlstrom, McNally) ignore or underplay the fact that false memories need not imply the absence of true memories, and, crucially, that recovered true memories are an established empirical phenomenon, as emphasized in the target article and by Gleaves and Smith. I note in section R4 that protracted therapies may be assumed to produce both recovery of true memories and "rich false memories" (Hayne et al.), which, however, may be negative false memories, that is, false memories that some event did not occur. Positing negative false-memory is, of course, another way of positing repression.

Another noteworthy trend (sect. R5), related probably to the current anti-Freudian climate in mainline psychology, is either to give Freud too much credit (e.g., make him responsible for the recovered therapy movement even though Freud withdrew his infantile seduction theory within ten years of its promulgation: Crews, McNally), or not to credit Freud enough (e.g., Crews, Kihlstrom) - as, for example, for his empirically based 
hypermnesic concentration technique and his prefiguring of experimental psychology's disproof of the effectiveness of hypnosis in producing hypermnesia.

Many of the commentaries focus on Freud - or on contemporary clinical framings of repression influenced by the recovered memory movement - and underplay or bypass TUTOR itself. Questions, however, are raised specifically about TUTOR: Is it too broad (Kihlstrom, Raz \& Fabrega)? Is it not broad enough (Freyd, Pintar \& Lynn)? Is it falsifiable (Raz \& Fabrega, Schmidt \& Van der Linden)? Is its incorporation of laboratory data justified and generalizable to the real world, especially when the laboratory stimuli are not emotional (Hayne et al.; Kihlstrom, McNally)? Because motive is crucial in defense, is it reasonable to separate the mechanism from the defense (Boden)? I try to address some of these concerns in section R6, realizing that ultimately the proof is in the pudding: Will TUTOR significantly enhance our understanding of repression, integrate dissociated areas of psychology, and lead to significant heuristic ramifications?

Questions are also raised about the two basic ways, according to TUTOR, that consciousness-lowering is effected: (1) through selective inhibition (avoidance, subtraction) of distressing material, or (2) through elaborative distortions (reconstructions) of such material. Both of these pillars of TUTOR are challenged - inhibition by Schmidt \& Van der Linden and constructive distortion by Esterson \& Ceci and Macmillan. These commentaries give me an opportunity (sect. R7) to develop in greater detail the profoundly constructivist nature of Freud's thinking, already obvious in - and perhaps a consequence of - his pre-psychoanalytic neuroscience work (e.g., his 1891 monograph, On aphasia [Freud 1891/ 1953]), and to suggest that a neurologically inspired constructivism, unlike Bartlett's, leads to a more nuanced position on memory, including the modern distinction between accessibility and availability. Macmillan, further, raises the question whether Ebbinghaus cognitively avoided the material on which he later tested himself. I try to show that this is a legitimate reading of Ebbinghaus, even though he never actually stated that he avoided thinking of the memory material. As to inhibition, I spell out my understanding of the concept and suggest that we need not question the viability of the inhibition construct in psychology.

Several commentators (Freyd, Pintar \& Lynn, Raz \& Fabrega, Shahar) - see section R8 - have enriched the discussion of repression by emphasizing interpersonal or social motives for subliminalizing troublesome realities through, for example, silence (Shahar) or the restructuring or reconstrual of distressing reality (e.g., Freyd, Smith). I suggest how these various social motives might be subsumed under TUTOR's two classes of repression. I also note that wider agreement would exist for repression (e.g., McNally 2003), if tendentious uninsightfulness, that is, denial, is incorporated into the concept, as I do in TUTOR and as do, ubiquitously, Freud and psychoanalytically oriented clinicians.

\section{R2. The semantic problem}

One conclusion that is supported by the commentaries is that much (though, of course, not all) of the controversy surrounding repression involves differences, mostly implicit, in the definition of repression. If, for example, defensive repression is defined as a process that is completely unconscious (e.g., Boag, Crews, Langnickel \& Markowitsch, Macmillan, Najmi \& Wegner); is not a "normal" memory phenomenon (e.g., Loftus \& Ketcham 1994; McNally 2003); is an operation that must yield complete amnesia (Hayne et al.); is restricted to episodic forgetting of the events surrounding external catastrophes, such as sexual abuse, torture, and war (e.g., Hayne et al.); or needs to involve sex (Macmillan) - then, probably, the concept will have been defined out of existence. If, on the other hand, as suggested in the target article, defensive repression is more or less (un)conscious and essentially the same as "suppression"; reveals itself differently in different cognitive media or states (as in jokes, dreams, daydreams); yields varying degrees of inaccessibility; includes tendentious uninsightfulness for latent contents, that is, denial (Freyd, Smith); depends both on types of stimuli and subjects (Boden, Freyd, Fujiwara \& Kinsbourne, Hermans et al.); varies over time (Fujiwara \& Kinsbourne); includes biological as well as social motives (Freyd, Pintar \& Lynn, Raz \& Fabrega, Shahar); and obeys the laws of normal memory (Anderson \& Levy, Gleaves, Medford \& David, Smith) - then repression may, after all, be an everyday fact.

Definitions should not, of course, be arbitrary, and they should follow traditional usage, especially the originator's conception of his idea, and be changed only because of explicitly justified scientific necessity. Thus, I cannot agree with Bonanno's suggestion that worrying too much about definitions is tantamount to engaging in "quibbles." No science is possible without some clearly understood agreement on what it is we are investigating - or debating. For example, Bonanno cites the (fine) work of Foa and colleagues showing that psychological techniques can actually reduce the upset of emotional events without eliminating accessibility to the facts of the events (Foa et al. 1995b). I specifically discuss in my target article (sect. 2, last para.) Freud's almost immediate realization (in 1894) that outright, complete inhibition of unbearable content was not the only technique employed by his patients, who were observed to deploy a variety of defensive transformations, including "isolation" or"intellectualization," in which factual and emotional components of ideas or memories are dissociated with, as Bonanno puts it, the "inhibition of their emotional component," resulting in memories that are "still accessible" but "less upsetting." Bonanno, however, is paraphrasing Freud, not disagreeing with him. Medford \& David's very interesting discussion of depersonalization disorder, in which "dampening of emotional responses" or "de-affectualization" is associated with neural circuits also involved in control subjects' suppression of emotional responses, may significantly advance our understanding of the "repression of experience rather than of knowledge." Moreover, other defensive transformations in addition to isolation - "de-affectualization" - for example, rationalization, projection, reversal, symbolization, denial - can take the sting out of intolerable reality while maintaining some connection to it (contra the assumption of some of the commentators - Hayne et al., Kihlstrom, McNally - that repression needs to produce complete amnesia). 
The "repression" in TUTOR essentially follows Freud's conception (in the wide sense), but formulates it in more explicit and fungible terms so as to link Freud's clinically derived ideas to the experimentally established psychological processes that underpin them.

Relevant to the semantic issue is an additional consideration: TUTOR is not only an elaboration of Freud's ideas but also a continuation of an interrupted integrative tradition in experimental psychology, most notably exemplified by Dollard and Miller's (1950) Personality and Psychotherapy, which developed a "liberalized" S-R psychology in which the forbidden "black box" of the radical behaviorists was reclaimed for psychology and repopulated with a variety of intervening covert (small-cap) s-r's assumed to follow the same laws as those established for the observable bracketing S-R's. In effect, these small s-r sequences, termed "cue-producing responses," were what we today call "cognition." Dollard and Miller experimentally probed overt approach and avoidance response gradients of rats to positively and negatively valenced stimuli (e.g., food, shock) and extracted from their findings an experimental psychology of approach-avoidance conflict which they applied to the central issue of conflict in psychoanalytic theory. Because free-associative fantasy - Freud's "microscope of mind" - shows that patients cognitively veer off from distressing ideas (at least sometimes), it was a goal of Dollard and Miller to demonstrate in the laboratory that the albino rat similarly could anticipate untoward consequences (e.g., shock) and try to escape the trajectory of his behavior before the ultimate shock stimulus (or anxiety) was actually experienced. The conditioning principle employed was stimulus generalization, in this case an anticipatory, temporal generalization; and so it was realized that the animal (rat or patient) did not actually have to avoid the nasty stimulus itself but - and perhaps without consciousness for the ultimate distressing event - inhibit early responses and veer off from progress in its direction. This gradient model of anticipatory avoidance can encompass the unconscious character that is often attributed to repression and which is reemphasized by some of the commentators (e.g., Boag, Crews, Langnickel \& Markowitsch, Macmillan, Najmi \& Wegner). Of course, by definition, gradients are not allor-none. Thus, two standard principles in experimental psychology, anticipatory avoidance and automatization (emphasized in the target article), militate against a categorical distinction between conscious-unconscious or suppression-repression. Kihlstrom asserts, however, drawing on Macmillan's authority, that "if the process is to have any chance of working" or "if the concept is to make any sense at all ... repression must always be unconscious." Why?

On the basis of their laboratory work and its extrapolation to the amnesias sometimes reported in the clinic or in war situations, Dollard and Miller's conclusion was that "there is no doubt about the reality of repression" (p. 201).

In a verbal learning variant of the Dollard and Miller analysis of repression, Glucksberg and King (1967) demonstrated that associatively linked verbal lists $(\mathrm{A} \rightarrow \mathrm{B} \rightarrow$ $\mathrm{C} \rightarrow \mathrm{D}$ ) in which $\mathrm{D}$ items were associated with shock, produced memory disruptions of $\mathrm{B}$ items because, presumably, subjects anticipatorily veer off from early stimuli (B) which are likely to lead eventually to the shock stimulus $(\mathrm{B} \rightarrow$ $\mathrm{C} \rightarrow \mathrm{D}$-shock). When a positive stimulus (money) was associated with D items, no such memory degradation was observed for B items. Glucksberg and King's study, "Motivated Forgetting Mediated by Implicit Verbal Chaining: A Laboratory Analog of Repression," confirmed their expectation "that forgetting does occur as a function of unpleasant associations" (p. 158). These authors don't worry about having failed to produce absolute amnesia; they are satisfied with statistically reliable forgetting effects. It is clear to me that Hayne et al. are imposing an arbitrary new criterion for repression which would have been puzzling to classic investigators - and one which is at variance with TUTOR's emphasis of "consciousness-lowering processes." From a theoretical standpoint, it may be wondered to what extent the anticipatory associative avoidance model of repression might be mapped onto the recent thought-inhibition studies of Anderson \& Levy and their colleagues. It certainly dovetails nicely with the experimental program of Sedikides \& Green on mnemic neglect and also with the truncation of memory search in the avoidant processing of autobiographical memory, discussed by Hermans et al. (see also the notion that response biases, $\beta$ 's, in sequential processing, can transmute into bona fide perception or memory effects as measured by $d^{\prime}$; Erdelyi 1974; Erdelyi et al. 1989).

\section{R3. The silence of experimental psychology}

The promising convergence of the experimental laboratory and the clinic came to an unexpected end in the late 1960s, ironically just at the point when the "cognitive revolution" was transforming psychology and yielding an intellectual climate that could readily assimilate the integrative program of Dollard and Miller. With occasional exceptions (such as the work of Holmes 1974), the field lapsed into an inexplicable silence on repression. In the definitive textbook on memory of the 1970s, Crowder (1976), the author's doctoral advisor at Yale and, later, friend, failed to include a single reference to repression in his index. Loftus (see Loftus \& Ketcham 1994), surveying the textbooks of the 1980s, observed a similar preternatural silence:

When I began to search through journal articles and textbooks for answers to these questions [about repression] I was confronted by an eerie silence. It was as if repression itself had gone to sleep in the nearly 100 years since Freud first proposed his theory of a defense mechanism that protects the conscious mind from painful feelings and experiences. (Loftus \& Ketcham 1994, p. 49)

The silence of scientific psychology on repression caused much harm, both outside and inside psychology. Just as Freud had not discovered the unconscious or repression, he also did not discover sex or false accusations of sexual abuse. Everyday people, confronted by crushing interpersonal tragedies in their lives, often entangled with sexual issues, turned to psychology and found that it had become an absentee landlord of its realm. Into this conceptual vacuum nonpsychologists (e.g., Bass \& Davis 1988), or scientifically untrained therapists, attempted to fill the breach and, despite their good intentions, garbled the science as well as the therapy. It is against this background of miscarried theory and practice that scientific psychologists, AWOL for a full generation, hurriedly marshaled a reaction. This reaction - perhaps overreaction - embraced a notion of 
repression that was not continuous or fully consistent with the classic conception. The contemporary semantic problem, in my view, partly resulted from this discontinuity in mainline psychology. The pre-silence "repression" was different from the post-silence "repression" of the deniers of repression (e.g., Bonanno, Hayne et al., Kihlstrom, McNally). TUTOR, essentially, attempts to put the classic tradition of repression back on contemporary scientific track.

\section{R4. The recovery of true and false memories}

Much of the contemporary debate about repressed memory is premised on the assumption (criticized by Gleaves) that recovered memories are invariably false. It is such an assumption that caused Loftus and Ketcham (1994), for example, to deduce that repressed memories were a "myth." If, however, we adopted signal detection theory (SDT) as a conceptual model for recovered memories, it would be evident that false alarms do not imply the absence of true hits. It is for this reason that in experimental psychology, for the past half century, sensitivity or memory $\left(d^{\prime}\right)$ is not indexed either by hit rate or false alarm rate but the isosensitive function (the "receiver operating characteristic" - ROC - curve) relating hit and false alarm rates, from which $d^{\prime}$ can be calculated.

Also, there has been a perennial neglect, only now beginning to be acknowledged by critics of repression - Hayne et al. - of the experimentally settled phenomenon of reminiscence (memory recovery) and hypermnesia (overall memory improvement) with repeated testing. Such hypermnesia effects with repeated testing raise questions about the apparent assumption of Bonanno, and also McNally (2003), that initially absent information that is produced in later interrogations indicates that the recovered information was merely withheld from earlier reports. The experimental and clinical literature (Erdelyi 1996; Gleaves, Smith) suggests that the recovered information may be, at least in part, recovered memory. Hayne et al. acknowledge that "Yes, subjects remembered more studied material if they attempted to recall it over and over again, but they never forgot that they had encountered the material in the first place." It is not necessary, however, to show complete amnesia - this is a false criterion - either in the laboratory or in the clinic (as in the Case of B.). Kihlstrom also seems to assume that amnesia needs to be complete and can be discounted in the case of partial amnesias. The critical point for TUTOR, as indicated in the target article, is "consciousness-lowering." Moreover, it should not be assumed that post-event implantations are the only story in protracted therapy. From the memory standpoint, why not view psychoanalytic therapy as a vast, multi-trial recall enterprise, lasting 50 minutes per "trial," repeated several times a week, for several years? It would be a worthy laboratory project - which could cut both ways - to determine the extent to which memories for complex experimentally presented stimuli (that are interesting and emotionally vital) could be recovered over such long periods of extended recall effort.

Although I have no doubt, along with Loftus and Freud, that conscious memory can be drastically distorted (and, incidentally, not just by experimental implantation but also by biologically prepared propensities of the subject), I do not think that Loftus herself has fully embraced the notion of "rich false memories," since that concept actually leads back to the phenomenon of repression for which Hayne et al. find a "continuing lack of scientific evidence." Memory distortions, after all, can be negative and not just positive. The existence of both positive and negative hallucinations is a standard notion in hypnosis and Pierre Janet (1889), in his famous case of Marie, claimed to have brought about a therapeutic resolution by suggestions that the distressing events from which Marie derived her symptoms had actually not taken place (or had been different). So, in effect, this was a false-memory implantation therapy in which the original event was reconstructed. The essential point is that a belief in memory implantation need not assume the sign of the memory: We may add false memories but also subtract-out true memories and thus repress them with or without the help of a therapist or experimenter. Hence, as Gleaves puts it, "if you believe in false memories, you apparently also believe in repression."

\section{R5. Too much or too little Freud}

Some commentators (Crews, Kihlstrom, McNally) emphasize that the harmful recovered-memory therapy movement (which began in the late 1980s) drew its inspiration and legitimacy from Freud's infantile seduction theory of hysteria (of around 1896). Freud, however, withdrew his theory within a decade, and throughout his career, emphasized his retraction of the error "that was almost fatal for the young science" (Freud 1914b/1958, p. 51). It seems to me neither reasonable nor fair to ignore or underplay this self-correction of Freud's and to shoulder him with the responsibility for the recoveredmemory therapy movement that unfolded some 80 years after his retraction. There is an attribution issue here, and I am inclined to place at least part of the responsibility on us experimental psychologists for allowing our discipline to fall silent on the issue and thus fail to inform - and to rein in - an untrammeled clinical movement. When it comes to blame, critics of Freud are too eager to lay responsibility on Freud. When it comes to palpable contributions, Freud's critics either ignore his contributions or disdain the contributions (Crews, Kihlstrom).

For example, Kihlstrom asserts in his commentary, "we did not need Freud to tell us that people remember better when they try harder." This sounds reasonable, only it fails to acknowledge how difficult it was for laboratory psychology to cinch this elementary point, which Ballard, independently of Freud, introduced into (English speaking) experimental psychology in 1913. For some half century, laboratory psychology kept getting both the concept and the stimulus wrong and concluded that the phenomenon was not reliable (see Erdelyi 1996). In this instance the experimental challenge was trivial, unlike in the case of defensive repression. It was only necessary to use effective stimuli, such as poetry or meaningful pictures instead of nonsense syllables. Thus, protracted failures of laboratory psychology to produce phenomena may reflect on laboratory psychology and not always on the phenomena. It was actually my experimental efforts to corroborate Freud's claim (in Breuer \& Freud 1895) that pressure or 
concentration could yield memory recovery - which it can - that brought back reminiscence and hypermnesia into mainline experimental psychology. Psychologists pervasively assumed for decades that memory inevitably declined with the passage of time, and so it was only natural for hypnosis researchers, for example, to assume that a second recall effort during (or after) hypnosis, which led to hypermnesia, meant that hypnosis was the effective agent (hence, hypnotic hypermnesia). With the laboratory demonstration of hypermnesia with just repeated recall, it was natural to wonder whether hypnosis played any part in hypnotic hypermnesia; perhaps concentration, pressure, or repeated effort was all that was required - as Freud (Breuer \& Freud 1895), drawing on his clinical observations, suggested, against prevailing wisdom. Modern experimental work in several laboratories (which I review in my 1996 book) has shown that Freud was right on both counts in this instance: (1) recall effort (concentration, repeated trials) yields hypermnesia, and (2) hypnosis adds nothing to this hypermnesia. Kihlstrom himself (Register \& Kihlstrom 1987), using my hypermnesia procedure based on Freud's, contributed to this experimental settling of the classic hypnotic hypermnesia problem.

\section{R6. The "theory" in TUTOR, falsifiability, and the bearing of laboratory data}

The most important theoretical feature of TUTOR is that it provides a framework for integrating the worlds of the clinic and the laboratory. From a more conventional standpoint - of theory providing a story that makes testable predictions - some commentators (Kihlstrom, Raz \& Fabrega) suggest that the theory is too broad and lacking in specificity. Kihlstrom, for example, suggests that I define repression "so broadly that the concept loses its meaning" or the "features that might make it interesting."

I devoted some effort in the historical section of my target article (sect. 2) to showing that throughout most of his psychological career, Freud used "repression" in a very wide sense which later (in 1926) he reformulated (not with the greatest consistency) as "defense mechanisms." In TUTOR I return to the wide conceptualization of repression and treat the narrow, inhibitory sense as one of the two basic subsets of general repression. I do not know how one might scientifically demonstrate that a theory is "interesting"; but repression in the wide sense, as in TUTOR, is typically considered interesting by students and psychologists - at least those who have not rejected the phenomenon. I agree with Boden that emotional motive is central to defensive repression, and motives - such as sex, aggression, grief, terror - invariably arise in clinical contexts (e.g., the Cases of B. and N.) and give defensive repression its wide interest value. (The logical separation between mechanism and motive in TUTOR does not imply a discounting of the importance of motive, though Boden correctly observes that it gets less play in TUTOR. The experimental laboratory is limited in the range and types of motives that it can study, and so the emphasis of TUTOR falls on the mechanisms - through which motives have their effects.)

Although TUTOR indeed has a broad sweep, the theory itself is rather simple. Its essential ingredient is the straightforward notion (articulated already in Erdelyi
1974; 1979) that bias begins at the beginning and only ends at the end of information processing. Thus, cognitive bias in the beginning can be readily instantiated by eyefixation behavior (e.g., Erdelyi 1974; Luborsky et al. 1990), as when, for example, a subject watching a horror movie with rapt attention averts his eyes or covers them when the material becomes too scary. At the other end of the information-processing continuum are response biases (response withholding, etc.), again easily demonstrated, since they are behaviors. The intervening cognitive mechanisms are more difficult to corroborate (e.g., encoding- and retrieval-inhibition), and were actually quite controversial for a period - indeed, the whole New Look debate, which presaged the repression debate of today, swirled around the less palpable cognitive biases. TUTOR, for the purpose of facilitating the integration of the clinic and the experimental laboratory, distinguishes between two classes of biased processing: inhibitory (avoidant, subtractive) processing and elaborative (additive, reconstructive) processing. The "theory" in TUTOR, as I set it out in section 3.1 of the target article, is not about some local mechanism, but, as Boden notes, about a pervasive theme of information processing: Distressing material will be subject to defensive consciousness-lowering through omissions and reconstructions across the information processing continuum.

Is this elementary proposal falsifiable (Raz \& Fabrega, Schmidt \& Van der Linden)? Although, at this broad level, the theory does not specify where, for a particular situation, particular stimulus, or particular individual, defensive bias is likely to manifest itself in the information-processing continuum (probably at several junctures) - just as one cannot specify which defense mechanisms (probably several) might be activated in a given case - TUTOR requires, at least in some region of the information processing continuum, that emotionality interact with consciousness (as Medford \& David suggest), and more specifically, that conscious accessibility or level be degraded - "liminalized" or "subliminalized" (Erdelyi \& Zizak 2004; Erdelyi \& Zizak, in preparation) to mitigate emotional distress.

Personality factors (Boden, Fujiwara \& Kinsbourne, Hermans et al.) may be conceived of as psychologically transducing the stimulus. The same objective magnitude of a given stimulus would be expected to yield different psychological magnitudes for different persons. A snake at a given distance will be more psychologically intense for a snake-phobic person than for a person who keeps snakes as pets. Obviously, mindless averaging of subjects' responses to an objectively set stimulus value, without concern for individual differences, may result in the canceling-out of otherwise powerful effects on consciousness.

There are other crucial - and, I think, fascinating - interactions to consider (Schredl points to one of these), which I purposely reined in from the discussion in my target article controversial enough in its simplest form. For example, the cognitive state or cognitive medium involved interacts with repression. What may be substantially subliminalized in recall, may be more accessible in jokes, dreams, or daydreams. Such interactions - between repression and the cognitive channel probed - leads, of course, to the heart of Freud's cognitive psychology.

Another important issue, raised in different ways by different commentators (e.g., McNally, Kihlstrom), is 
the question of generalizability. Thus, is the laboratory evidence for selective inhibition, reconstructive elaboration, and memory recovery irrelevant or peripheral because it does not apply to the real world (Kihlstrom)?

Generalability is, of course, always a central scientific question and inevitably, no matter how successful the theory, there is always a limit to it. I agree, therefore, with some of the commentators' concerns about generalability, noting, however, that the answer must be empirical: We must experimentally probe the extent to which the everyday mechanisms of inhibition and elaboration which, according to TUTOR, generate all the manifestations of repression classically reported in the clinic, can really account for responses to the hurly burly of the real world, from betrayal by our loved ones to torture and death in war and genocide. Before we generalize too little, however, and allow the confusing proliferation of ad hoc hypotheses to account for local phenomena, we should give every chance possible to the simple theories that are based on bedrock experimental foundations. For example - and this is only one - the propensity to distinguish between repression and dissociation (e.g., Kunzendorf, Raz \& Fabrega), and to give special status to mental "fragmentation" observed in trauma, is not necessary. These phenomena can be accommodated by the widespread observation (e.g., Freud 1891/1953; 1893/1966; 1917/1961/1963; Janet 1889; Pötzl 1917; Fisher 1956; 1988; Erdelyi 1996; Werner 1956) of the fragmentation of speech, perception, memory - and sleep consciousness (dreams) - that results from the exhaustion of mental resources (consequent to brain damage, tachistoscopic degradation, psychological state (fatigue, dream sleep), hereditary factors, and cognitive masking (though note should be taken of Gleaves' and Medford \& David's caution that the evidence for cognitive masking is more nuanced than I supposed).

For practical and ethical reasons, there are stringent limits to the extent to which we can probe the perimeters of our laboratory-based principles, and so it makes good sense to assess, as systematically as possible, the phenomena of the real world. It is a mistake, however, to expect the exquisite manipulations and controls possible in the laboratory to be implemented in the outside world and then to use such unavoidable imperfections to dismiss the viability of repression. It is not that the methodological scruples emphasized are not important. Amnesias resulting from brain traumas should not be confused with functional amnesias (however, see Markowitsch 1999), but it would be unrealistic to expect crisp neurological diagnoses on the battlefield; indeed, standard neurological workups are not irreproachable (or final). Perhaps a necessary step for combining the rigor of the laboratory with the richness of the real world is for the experimental laboratory to move to the clinic - the marriage-therapy office or the oncology clinic, for example - and exactingly instantiate and describe the course of defensive repression for different levels and kinds of stimuli (Gleaves, Freyd, Medford \& David) and different types of persons (Boden, Fujiwara \& Kinsbourne, Hermans et al.).

There is also the mind-brain connection to consider. Mental states produce organic changes, just as software produces changes in nuts-and-bolts hardware. Some of the commentators - Medford \& David, Fujiwara \& Kinsbourne, Anderson \& Levy - significantly enrich the target article by pointing to some of these neuroscience underpinnings (though we must be cautious, as McNally [2003] shows, about too enthusiastically embracing dramatic first-blush evidence of neurological substrates, as, for example, the empirical claim that traumatic stress leads to the shrinkage of the hippocampus see especially the MRI twin study of Gilbertson et al. [2002]). Markowitsch (1999), however, in his neuroimaging work on selective memory disturbances has gone as far as to suggest "the necessity to question the dichotomy between organic and psychological amnesia" (p. 568). (It is a pity Langnickel \& Markowitsch do not also question the dichotomies they impute to psychoanalysis. I do discuss in my target article some of the issues about which Langnickel \& Markowitsch make authoritative assertions, but regrettably they do not engage the relevant material, especially section 2 of TUTOR.)

\section{R7. Inhibition and distortion}

Several of the commentators challenge one or the other of the two pillars of TUTOR, either the inhibition/subtraction component (Schmidt \& Van der Linden) or the elaborative distortion part (Esterson \& Ceci, Macmillan). These challenges are both relevant and useful, since refutation of either component of TUTOR would yield a far less general theoretical framework, and refutation of both would, of course, lead to the outright collapse of the theory.

I will begin with the elaborative foundation of TUTOR, which is that accessible memory is pervasively distorted, and that Freud and Bartlett agree in this respect but simply emphasize a different motive for distortion - cognitive needs for Bartlett (logic, expectations, cultural habits) and emotional needs for Freud (wishes, defenses). The constructivist tradition, as William Brewer (1984) showed, was the prevalent view in Continental Europe which Bartlett (1932) succeeded in finally imposing on a recalcitrant Anglo-American laboratory psychology. Freud belongs to the earlier Continental European tradition. Except for the motive issue (and the depth issue - see below), I don't think that Freud's and Bartlett's positions are discriminable. Consider the following quotes:

(a) "The tendency of the human mind to see everything connectedly is so strong that in memory it unwittingly fills in any lack of coherence there may be...."

(b) "Thus it may easily happen that waking consciousness unwittingly makes interpolations in the memory of a dream: We persuade ourselves that we have dreamt all kinds of things that were not contained in the actual dreams."

(c) "Total forgetfulness is not serious; but partial forgetfulness is treacherous. For if we then proceed to give an account of what we have not forgotten, we are liable to fill in from our imagination the incoherent and disjointed fragments furnished by memory. ... We unwittingly become creative artists; and the tale, if it is repeated from time to time, imposes itself on its author's own belief, and he ends up by offering it in good faith as an authentic fact duly and legitimately established."

(d) "In our efforts at making an intelligible pattern of the sense-impressions that are offered to us, we often 
fall into the strangest errors or even falsify the truth about the material before us."

(e) The observer "fills up the gaps of his perception.... He may do this without being in the least aware that he is either supplementing or falsifying the data of perception."

All five quotes seem to be giving expression to the same basic constructivist theme and are listed in chronological order: (a), (b), and (c), respectively, are from 1855 (Jessen), 1877 (Strümpell), and 1895 (Egger), and are used by Freud (1900/1953, p. 46) in his review of constructivist notions about dream recall (or memory in general); (d) is Freud's statement (1900/1953, p. 499); and (e) is Bartlett's (1932, p. 14).

In one respect, perhaps because of his neuroscience background, Freud has a more nuanced position on memory than Bartlett, whose notion of memory is essentially one-dimensional: What you get in remembering is memory, period. For Freud, memory is multilayered; what is "inaccessible" may nevertheless be available. The distortions operate on accessible memory, without necessarily obviating the availability of more veridical information. Thus, by ignoring the possibility of unconscious memories, which may be more accurate than their conscious transforms, Bartlett, in one of his few references to Freud, misstates Freud's position: "we find Freud developing the view that memories form a static mass" (Bartlett 1932, p. 15). For the neurologist Freud (e.g., 1891/1953), concerned with aphasias and paraphasias (representational derailments in which, for example, a semantically related word replaces the original), it is obvious that brain structure and damage do not yield an isomorphic conscious experience. Indeed - and this is one of Freud's great constructivist insights (along with Charcot's) - hysterical symptoms are psychological reconstructions of neurological or "organic" reality. For example: "Hysteria is ignorant of the distribution of the nerves .... It takes the organs in the ordinary, popular sense of the names they bear: the leg is the leg ... (Freud 1893/1966, p. 169). Hysterical paralysis, therefore, is an "alteration" of the neurological truth by the narrative psychological truth - the "conception, the idea" (Freud $1893 / 1966$, p. 170) of, for example, "leg."

Macmillan suggests that my entries in Table 1 of the target article do not faithfully reflect homologies between Freudian and Bartlettian distortions. I am puzzled, since this is a straightforward matter, and to my best understanding Macmillan is misreading both Bartlett and Freud. I see no problem in the equivalence of condensation in the two systems. Both Freud and Bartlett emphasize the "shortening" resulting from condensation: By "CONDENSATION ... we mean to convey the fact that the content of the manifest dream is less rich than that of the latent thoughts.... [It is] a kind of abbreviated translation of the latter" (Freud 1917/1961/1963); condensation reflects a "tendency to compression... and therefore economy" (Freud 1905/1958). Quotes to this effect could be easily multiplied. Bartlett, for his part, describes many instances in which two elements of a stimulus - geometric pictures, "The War of the Ghosts" - are merged into a single element. For example, the idea that "He was dead" and "something black came out of his mouth" is rendered by one subject as "The black man is dead" (p. 72). I do not understand why elaborations or secondary revision constitute a seriously "misleading comparison." For Freud, for example, "the dream does not merely reproduce the stimulus, but elaborates it, plays upon it, fits it into a context, or replaces it by something else" (Freud 1917/ 1961/1963, p. 86). For Bartlett (1932) "accuracy of reproduction, in a literal sense, is the rare exception and not the rule.... In long-distance remembering, elaboration becomes rather more common ... and there may be increasing importation, or invention" (p. 93). I believe that Macmillan is mistaken here, as he is in claiming a logical problem on my part, because, as can be verified by checking my target article (e.g., sect. 3.1), I do not claim "that everything that is kept out of consciousness is kept out because of repression." Inaccessibility due to brain damage, for example, is not the same as inaccessibility due to repression, and defensive repression is only a subset of repression in TUTOR.

Macmillan also raises the question, which he thinks is crucial for Freud, "Why was it that only sexual ideas were repressed?" which he claims is dismissed by me "as an isolated and ambiguous titbit [sic?]." Sex, per se, is not an issue for TUTOR nor, arguably, for Freud with respect to repression: "There is therefore no connection with sexuality in the concept of 'repression'; please mark this very carefully. It denotes a purely psychological process" (Freud 1917/1961/1963, p. 299).

The second chapter of Bartlett's Remembering, is, as Macmillan notes, about perceptual constructivism. Indeed, it was this chapter that initially cued me to the overlap between Bartlett and Freud. The "New Look" in perception, which I mention in my target article, precisely concerned the interposition of internal structures, whether expectations or defenses, between the stimulus and the percept, and began as an elaboration of the Bartlett program. Most relevant, however, was the duplication of phenomena from clinical neurology in laboratory demonstrations of counterpart effects by Otto Pötzl, a professor of psychiatry and neurology at the University of Vienna and a member of Freud's psychoanalytic circle. Pötzl had observed some curious reconstructive phenomena with some of his brain-damaged patients, for example, one Obszut, who had managed to sustain an injury to his visual cortex in a duel. Obszut had lost central vision, though he had some spared peripheral vision. What intrigued Pötzl was that central stimuli, to which Obszut was blind, sometimes turned up later, in transformed versions, in ongoing peripheral percepts. Pötzl wondered whether he could experimentally produce such recoveries in dreams for stimuli that had been rendered tachistoscopically subliminal during the day. Pötzl succeeded in showing the effect with some dozen subjects and reported his findings to the Vienna Psychoanalytic Society and published them in 1917 (see Pötzl 1917).

Charles Fisher (e.g., 1956; 1988), a psychoanalyst, extensively replicated the Pötzl findings in dreams, daydreams, and free-associative fantasy. Although, as Schredl notes, the methodology was not unassailable, the basic effects can be easily replicated in standard, controlled laboratory studies (e.g., Haber \& Erdelyi 1967; for a review, see Erdelyi 1996). The Pötzl-Fisher effects are, in fact, sublimely reliable and could easily serve as classroom demonstrations of Freudian reconstructions. Fisher often refers to these transformations, which are indistinguishable from the ones that Bartlett 
emphasizes, including: displacements, fusions/condensations, fragmentation, symbolism, mirror images/reversals, multiplication of items (polyopia), and rotations and spatial translocations. Because these are the types of distortions and transformations observed not only in braindamaged people or tachistoscopic subjects but also in the dream-content of normal persons - as well as in jokes, fantasy, art, and psychosis - it may be concluded, along Freudian lines, that resource-depletion (a problem of mental "economics," in Freud's parlance) yields the same distortions, whether these are brought about by "gizmo" subliminalization (Erdelyi \& Zizak 2004; Erdelyi \& Zizak, in preparation) - due to brain damage or to a tachistoscope - or by psychological subliminalization resulting from forgetting (Ebbinghaus), elaborative distortions (Bartlett, Freud), "cognitive load" (Najmi \& Wegner), and so on. It is in the big picture, the gestalt, and not in the less remarkable parts (on which critics of repression often choose to focus) that the great contributions of Sigmund Freud to cognitive psychology can be found (Erdelyi 1999).

If the Bartlett-Freud connection is reasonably in the ballpark, then we no longer need to persevere, as Pintar \& Lynn note, in the one-track construal of repression as merely degradation of episodic memory for traumatic experiences. The classic literature, especially that of Freud and Dollard-Miller, never was remotely as narrow as deniers of repression would have it (e.g., Crews, Kihlstrom, Hayne et al., McNally, Macmillan), involving, instead, a wide-angle approach that included all of the defensive distortions under the heading of "repression" (in the wide sense).

As to the inhibition issue raised by Schmidt \& Van der Linden and the question of Macmillan's regarding whether Ebbinghaus really "intentionally excluded" his target memories from awareness, the short answer is that we are struggling here with semantics - although, as Smith cautions, these may subsume legitimate conceptual issues. In the case of Ebbinghaus (1885/1964), he memorized his nonsense syllables to a perfect criterion and then "left [the memorized items] to themselves" (pp. 62, 65) during the retention interval. I take this to mean that Ebbinghaus avoided thinking of the list, or excluded the list from consciousness. If this is not a reasonable construal (what would be a reasonable alternative?), we might carry out the following experimental study: One group memorizes the nonsense syllables and is then told that the items memorized are to be "left to themselves" (Ebbinghaus, p. 65), and the other group is treated identically but told "not to think" of the material. I suspect both instructions would yield the classic Ebbinghaus outcome: "All sorts of ideas, if left to themselves, are gradually forgotten” (p. 62). Also, Najmi \& Wegner need to confront explicitly the fact of Ebbinghausian forgetting. If instead of the sub-span "white bears," we asked subjects not to think of an Ebbinghaus list of nonsense syllables or, even, "The War of the Ghosts," the outcome would be reduced memory, not rebound.

With regard to "the existence of inhibitory processes," Boag suggests, as would most psychologists, that they are "incontrovertible." Schmidt \& Van der Linden, however, raise the question whether "inhibition" needs to be invoked, or even accepted as a valid construct. What really is the issue? Would "subtraction" be an acceptable substitute metaphor? Terms like "inhibition," "automatization," and "implicit" are often treated in cognitive psychology as formal scientific concepts when, in fact, they are "pretheoretic" (Erdelyi 2004a; 2004b), terms that largely carry their meaning through habit or intuition. How would we know whether a neurotransmitter, $x$, was excitatory or inhibitory, especially if $x$, as in many cases, could be both, depending on the locus of its operation? Would we not operationalize $x$ 's nature by its effects on the postsynaptic neurons? If the probability of their firing is elevated, $x$ is excitatory; if degraded, $x$ is inhibitory. Thus, as I understand it, inhibition is an operation which degrades (subtracts from) the output of the system/unit upon which it is operating. If avoidance of consciousness for some target material results in degraded accessibility for this material, then the avoidance corresponds to inhibition. By this view, "selective attention," to which Schmidt \& Van der Linden apparently subscribe, necessarily implies inhibition (as in dichotic listening) since the rejected channel rapidly becomes inaccessible.

\section{R8. Wider contexts, including social groups}

I was surprised by the number of commentators who sought to supplement TUTOR with an emphasis of "social motives," "cultural" effects, or "interpersonal factors" (Freyd, Pintar \& Lynn, Raz \& Fabrega, Shahar). Whereas some commentators criticized TUTOR for being too broad (e.g., Kihlstrom, Raz \& Fabrega), others noted that my "focus is still too narrow" (Pintar \& Lynn) or that I "neglected a crucial aspect of repression - the social psychological forces or motives" (Freyd). I agree with Pintar \& Lynn and Freyd. Although it might not be obvious on first blush, I took an exceedingly conservative approach in TUTOR, in which I stressed two highly worked-through and accepted principles in experimental psychology - selective inhibition and reconstructive distortion - as a foundation on which to build the future scientific edifice of repression. If it can be accepted that inhibition and reconstructive distortion can generate all the classic manifestations of repression, as I think they can, then we can move into the next, truly exciting phase of the project, to expand its reach and depth.

This future elaboration of repression almost surely will move, as some of the commentators have already adumbrated, in two opposite directions - one inwardly, toward biological underpinnings and motives (as in, for example, Fujiwara and Kinsbourne's temporal dynamics of hormone states), and the other, outwardly, toward the context of family, group, and society.

Some of the social motives can be subsumed under the inhibitory component of TUTOR, including "silencing theory" and interpersonal "oppression" emphasized by Shahar, and others under the elaborative distortion component, for example, the "narrative pressures" imposed by "social realities" discussed by Pintar \& Lynn. Freyd's betrayal trauma theory (BTT) provides a prototype of the biologically invested social contexts in which "social motives may even trump emotional motives." Freyd's Figure 2 dramatically illustrates how interpersonal relations may moderate the effects of abuse on consciousness-lowering, and her Figure 1 articulates explicitly my 
References/Erdelyi: The unified theory of repression

brief suggestion in the target article (based on her work) that emotional intensity is not a simple dimension.

Importantly, denial - conceived of as tendentious uninsightfulness - with which the social-motive commentators readily resonated (Freyd, Pintar \& Lynn, Shahar), blows the repression cat out of the bag, since even severe critics of repression, such as McNally (2003), acknowledge that, apart from episodic memory for past abuse, the construal of the relevant events may change over time. Such reconstrual - "insight," "restructuring" which may be thought of as the other side of denial (Erdelyi 1985), is modulated, as Smith notes, by "affective consequences": the extent to which insight/denial produces "disturbing realizations" or "relief." Laboratory psychology has typically ignored the interpretation issue in repression and focused excessively on the episodic details of past events; that is, it has focused on the manifest (surface) content of events at the expense of their latent (deep) semantic contents. It would be easy to disprove that there is motion in motion pictures, yet it is our "interpretation" - our illusion - that is the clinching reality, not the frame-by-frame auditing of stimulus sequences.

The "repression" that is characteristically encountered in case histories, Freud's for example - or revealed in relatively disinhibited (de-repressed) cognitive media, such as jokes, dreams, and daydreams - are typically about latent meanings and not about absolute amnesias overtaking a traumatized person. The repressions we encounter ubiquitously in everyday life involve the tendentious extraction (or failures of extraction) of meanings, as in Freyd's betrayal blindness - exactly the kind of "blindness" entailed in denial. For this reason, I have suggested in the past (e.g., Erdelyi \& Goldberg 1979) that love-relations might be the place to search for meaning-based repression and have pointed here to the clinical setting of marriage-therapy as a real-world realm to which the experimental laboratory might redeploy. When I discuss with students or colleagues the drastic distortions of reality that love-relations (and their rupture) instigate, I never get skeptical arguments but rather nodding recognition of the ravages of reality that "the malady of love" visits upon us.

\section{R9. Conclusion}

TUTOR apart, the present dialogue in $B B S$ is an important development for repression. The appearance of so many energetic views in one "sandwich" (as I have begun to think of it) forces us to confront disparate ideas and criticisms which, for some decades, mainline psychology has mostly avoided under the cover of silence, or evaded by euphemistic circumventions. The critics have some strong points, but their positions, as I have tried to show, also have serious weaknesses. Falsifiability, as some commentators have noted, is an important consideration in the scientific enterprise, but falsifiability applies not only to the much-falsified Freud, but to the critics of Freud as well. Perhaps the time has come - for all involved - to revise positions that are no longer viable. Scientific progress - and fascinating new ideas - will be the reward.

\section{References}

[The letters " $a$ " and " $r$ " before author's initials stand for target article and response references, respectively.]

Abramowitz, J. S., Tolin, D. F. \& Street, G. P. (2001) Paradoxical effects of thought suppression: A meta-analysis of controlled studies. Clinical Psychology Review 21:683-703. [SN]

Alexander, C. N., Langer, E. J., Newman, R. I., Chandler, H. M. \& Davies, J. L. (1989) Transcendental meditation, mindfulness, and longevity: An experimental study with the elderly. Journal of Personality and Social Psychology 57(6):950-64. [AR]

American Psychiatric Association (1987) Diagnostic and statistical manual of mental disorders, 3rd edition (revised). American Psychiatric Association. [aMHE] (2000) Diagnostic and statistical manual of mental disorders, 4th edition. (revised). American Psychiatric Association. [aMHE]

Amir, N., Stafford, J., Freshman, M. S. \& Foa, E. B. (1998) Relationship between trauma narratives and trauma pathology. Journal of Traumatic Stress 11:385-93. [JP]

Anderson, J. (1927/1962) The knower and the known. Proceedings of the Aristotelian Society 27:61-84. (1962 reprint in: Studies in empirical philosophy, pp. 27-40. Angus \& Robertson.) [SB]

(1930/1962) Realism and some of its critics. Australasian Journal of Psychology and Philosophy 8:113-134. (1962 reprint in: Studies in empirical philosophy, pp. 41-59. Angus \& Robertson.) [SB]

Anderson, M. C. (2001) Active forgetting: Evidence for functional inhibition as a source of memory failure. Journal of Aggression, Maltreatment, and Trauma 4:185-210. Also in: Trauma and cognitive science: A meeting of minds, science, and human experience, ed. J. F. Freyd \& A. P. DePrince, pp. 185-210. Howarth Press. [MCA, aMHE, JFK]

(2003) Rethinking interference theory: Executive control and the mechanisms of forgetting. Journal of Memory and Language 49:415-45. [MCA]

(2006) Repression: A cognitive neuroscience approach. In: Psychoanalysis and neuroscience, ed. M. Mancia. Springer. [MCA]

Anderson, M. C., Bjork, R. A. \& Bjork, E. L. (1994) Remembering can cause forgetting: Retrieval dynamics in long-term memory. Journal of Experimental Psychology: Learning, Memory, and Cognition 20:1063-87. [MCA, SMS]

Anderson, M. C. \& Green, C. (2001) Suppressing unwanted memories by executive control. Nature 410:366-69. [MCA, HH, JFK, SN, SMS, aMHE]

Anderson, M. C. \& Kuhl, B. A. (2004) Inhibitory control and the suppression of unpleasant events. Paper presented at the 45th Annual Meeting of the Psychonomic Society, Minneapolis, MN, November 18-21. [MCA]

Anderson, M. C. \& Levy, B. (2002) Repression can (and should) be studied empirically. Trends in Cognitive Sciences 6:502-503. [MCA, JFK]

Anderson, M. C., Ochsner, K. N., Kuhl, B., Cooper, J., Robertson, E., Gabrieli, S. W., Glover, G. H. \& Gabrieli, J. D. E. (2004) Neural systems underlying the suppression of unwanted memories. Science 303:232-35. [MCA, aMHE, HH]

Ansfield, M. E., Wegner, D. M. \& Bowser, R. (1996) Ironic effects of sleep urgency. Behaviour Research and Therapy 34:523-31. [SN]

Anspaugh, K. (1995) Repression or suppression? Freud's interpretation of the dreams of Irma's injection. Psychoanalytic Review 82:427-41. [SB]

Ashley, A. \& Holtgraves, T. (2003) Repressors and memory: Effects of self-deception, impression management, and mood. Journal of Research in Personality 37(4):284-96. [JMB]

Baars, B. J. (2002) The conscious access hypothesis: Origins and recent evidence. Trends in Cognitive Sciences 6(1):47-52. [JMB]

Badcock, C. (2000) Evolutionary psychology: A critical introduction. Polity Press/ Blackwell. [aMHE]

Baddeley, A. D. (1976) The psychology of memory. Basic Books. [aMHE]

Bahrick, H. P. \& Hall, L. K. (1991) Preventive and corrective maintenance of access to knowledge. Applied Cognitive Psychology 5:1-18. [aMHE]

(1993) Long intervals between tests can yield hypermnesia: Comments on Wheeler and Roediger. Psychological Science 4:206-207. [aMHE]

Baker, A. J. (1986) Australian realism. Cambridge University Press. [SB]

Baker, D., Hunter, E., Lawrence, E., Medford, N., Patel, M., Senior, C., Sierra, M., Lambert, M., Phillips, M. \& David, A. S. (2003) Depersonalisation disorder: Clinical features of 204 cases. British Journal of Psychiatry 182:428-33. [NM]

Ballard, P. B. (1913) Oblivescence and reminiscence. British Journal of Psychology (Monograph Supplements) 1(2):82. [aMHE]

Banks, W. P. \& Pezdek, K., eds. (1994/1995) The recovered memory/false memory syndrome debate. Holt, Rinehart \& Winston. Special issue of Consciousness and Cognition, vols. 3 and 4(3/4). [aMHE]

Bargh, J. A. \& Chartrand, T. L. (1999) The unbearable automaticity of being. American Psychologist 54:462-479. [aMHE]

Barnier, A. J., Conway, M. A., Mayoh, L., Speyer, J. \& Avizmil, O. (in press) Directed forgetting of autobiographical memories. Journal of Experimental Psychology: General. [MCA] 
Barnier, A. J., Hung, L. \& Conway, M. A. (2004) Retrieval-induced forgetting of emotional and unemotional autobiographical memories. Cognition and Emotion 18:457-77. [MCA]

Bartlett, F. C. (1932) Remembering: A study in experimental and social psychology Cambridge University Press. [JFK, MM, arMHE]

Bass, E. \& Davis, L. (1988) The courage to heal: A guide for woman survivors of child sexual abuse. Harper \& Row. [rMHE]

Bass, E. \& Davis, L. (1994) The courage to heal. HarperCollins. [aMHE]

Bateson, G. (1972) Steps to an ecology of mind: Collected essays in anthropology, psychiatry, evolution, and epistemology. University of Chicago Press. [JP]

Baumeister, R. F., Dale, K. \& Sommer, K. L. (1998) Freudian defense mechanisms and empirical findings in modern social psychology: Reaction formation, projection, displacement, undoing, isolation, sublimation, and denial. In: Defense mechanisms in contemporary personality research, ed. P. B. Cramer \& K. Davidson. Journal of Personality [Special Issue] 66:1081-124. [aMHE]

Bechara, A., Tranel, D., Damasio, H., Adolphs, R., Rockland, C. \& Damasio, A. R. (1995) Double dissociation of conditioning and declarative knowledge relative to the amygdala and hippocampus in humans. Science 269:1115-18. [aMHE]

Beck, J. G., Gudmundsdottir, B., Palyo, S. A., Miller, L. M. \& Grant, D. M. (2006) Rebound effects following deliberate thought suppression: Does PTSD make a difference? Behavior Therapy 37:170-80. [RJM]

Becker-Blease, K. A., Freyd, J. J. \& Pears, K. C. (2004) Preschoolers' memory for threatening information depends on trauma history and attentional context: Implications for the development of dissociation. Journal of Trauma and Dissociation 5:113-31. [JJF]

Beevers, C. G., Wenzlaff, R. M., Hayes, A. M. \& Scott, W. D. (1999) Depression and the ironic effects of thought suppression: Therapeutic strategies for improving mental control. Clinical Psychology: Science and Practice $6: 133-48 . \quad[\mathrm{SN}]$

Bergstein, J. (2006) Recognition hypermnesia. Unpublished doctoral dissertation, The City University of New York. [aMHE]

Bergstein, J. \& Erdelyi, M. H. (in preparation) Recognition hypermnesia: How to get it. [aMHE]

Billig, M. (1999) Freudian repression: Conversation creating the unconscious. Cambridge University Press. [aMHE]

Bjork, E. L. \& Bjork, R. A. (2003) Intentional forgetting can increase, not decrease, residual influences of to-be-forgotten information. Journal of Experimental Psychology: Learning, Memory, and Cognition 29:524-31. [MCA]

Bjork, E. L., Bjork, R. A. \& Anderson, M. C. (1998) Varieties of goal-directed forgetting. In: Intentional forgetting: Interdisciplinary approaches, ed. J. M. Golding \& C. M. MacLeod, pp. 103-137. Erlbaum. [MCA]

Bjork, R. A. (1978) The updating of human memory. In: The psychology of learning and motivation, ed. G. H. Bower, pp. 235-59. Academic Press. [JFK]

(1989) Retrieval inhibition as an adaptive mechanism in human memory. In: Varieties of memory and consciousness: Essays in honour of Endel Tulving, ed. H. L. Roediger \& F. I. M. Craik, pp. 309-30. Erlbaum. [aMHE]

Blagov, P. \& Singer, J. A. (2004) Four dimensions of self-defining memories (content, specificity, meaning and affect) and their relationship to self-restraint, distress and repressive defensiveness. Journal of Personality 72:437-59. [DH]

Bliss, E. L. (1986) Multiple personality, allied disorders, and hypnosis. Oxford University Press. [RGK]

(1988) A reexamination of Freud's basic concepts from studies of multiple personality disorder. Dissociation: Progress in the Dissociative Disorders $1(3): 36-40 . \quad$ [RGK]

Blondel, C. (1914) La conscience morbide: Essai de psychopathologie générale. Librairie F. Alcan. [JP]

Boag, S. (2006) Freudian repression, the common view, and pathological science. Review of General Psychology 10:74-86. [SB]

(in press) Realism, self-deception and the logical paradox of repression. Theory and Psychology. [SB]

Boden, J. M. \& Baumeister, R. F. (1997) Repressive coping: Distraction using pleasant thoughts and memories. Journal of Personality and Social Psychology 73(1):45-62. [JMB]

Boden, J. M. \& Dale, K. L. (2001) Cognitive and affective consequences of repressive coping. Current Psychology: Developmental, Learning, Personality, Social 20(2):122-37. [JMB]

Bonanno, G. A., Keltner, D., Holen, A. \& Horowitz, M. J. (1995) When avoiding unpleasant emotions might not be such a bad thing: Verbal-autonomic response dissociation and midlife conjugal bereavement. Journal of Personality and Social Psychology 69:975-89. [EF]

Bonanno, G. A., Keltner, D., Noll, J. G., Putnam, F. W., Trickett, P., LeJeune, J. \& Anderson, C. (2002) When the face reveals what words do not: Facial expressions of emotion, smiling, and the willingness to disclose childhood sexual abuse. Journal of Personality and Social Psychology 83:94-110. [GAB]
Bornstein, R. F. \& Pittman, T. S., eds. (1992) Perception without awareness: Cognitive, clinical, and social perspectives. Guilford Press. [aMHE] Brenner, C. B. (1973) An elementary textbook of psychoanalysis. Anchor. [aMHE]

Breuer, J. \& Freud, S. (1885) Studien über hysterie. Franz Deuticke. [FC] Breuer, J. \& Freud, S. (1893/1955) On the psychical mechanism of hysterical phenomena: Preliminary communication, trans. A. Strachey \& J. Strachey. In: The standard edition of the complete psychological works of Sigmund Freud, vol. 2, ed. J. Strachey. Hogarth Press. [aMHE]

Breuer, J. \& Freud, S. (1895/1955) Studies on hysteria. In: The standard edition of the complete psychological works of Sigmund Freud, vol. 2, ed. \& trans. J. Strachey, pp. 19-305. Hogarth Press. [MM, arMHE]

Brewer, W. F. (1984) The nature and function of schemas. In: Handbook of social cognition, vol. 1, ed. R. S. Wyer, Jr. \& T. K. Srull, pp. 119-60. Erlbaum. [rMHE]

Brewin, C. R. (2003) Post-traumatic stress disorder: Malady or myth. Yale University Press. [aMHE]

Brierley, B., Medford, N. \& David, A. S. (in press) Emotional memory: Separating content and context. Cognition and Emotion. [NM]

Brosschot, J. F., De Ruiter, C. \& Kindt, M. (1999) Recall and recognition of threatening, pleasant, and neutral words in repressors. European Journal of Personality 13(1):1-14. [JMB]

Brown, D., Scheflin, A. W. \& Hammond, D. C. (1998) Memory, trauma treatment, and the law. Norton. [aMHE]

Brown, D., Scheflin, A. W. \& Whitefield, C. L. (1999) Recovered memories: The current weight of the evidence in science and the courts. Journal of Psychiatry and Law 27:5-156. [aMHE]

Bruck, M., Ceci, S. J. \& Hembrooke, H. (2002) The nature of children's true and false narratives. Developmental Review 22:520-54. [AE]

Bulevich, J. B., Roediger, H. L. III, Balota, D. A. \& Butler, A. B. (in press) Failures to find suppression of episodic memories in the think/no-think paradigm. Memory and Cognition. [HH]

Burke, A., Heuer, F. \& Reisburg, D. (1992) Remembering emotional events. Memory and Cognition 20:277-90. [NM]

Bush, G., Luu, P. \& Posner, M. I. (2000) Cognitive and emotional influences in anterior cingulate cortex. Trends in Cognitive Sciences 4(6):215-22. [AR]

Buxton, C. E. (1943) The status of research in reminiscence. Psychological Bulletin 40:313-40. [aMHE]

Byrne, D. (1961) The repression-sensitization scale. Rationale, reliability, and validity. Journal of Personality 29:334-49. [aMHE]

Calvo, M. G. \& Eysenck, M. W. (2000) Early vigilance and late avoidance of threat processing: Repressive coping versus low/high anxiety. Cognition and Emotion 14:763-87. [aMHE]

Campbell, S. (2003) Relational remembering: Rethinking the memory wars. Rowman \& Littlefield. [JJF]

Cardinal, R. N., Parkinson, J. A., Hall, J. \& Everitt, B. J. (2002) Emotion and motivation: The role of the amygdala, ventral striatum, and prefrontal cortex. Neuroscience and Biobehavioral Reviews 26:321-52. [SB]

Cartwright, R. D., Bernick, N., Borowitz, G. \& Kling, A. (1969) Effect of an erotic on the sleep and dreams of young men. Archives of General Psychiatry 20:262-71. [MS]

Ceci, S., Huffman, M. L. C., Smith, E. \& Loftus, E. (1996) Repeatedly thinking about a non-event: Source misattributions among preschoolers. In: The recovered memory/ false memory debate, ed. K. Pezdek \& W. P. Banks, pp. 225-44. Academic Press. [aMHE]

Chu, J. A. \& Dill, D. L. (1990) Dissociative symptoms in relation to childhood physical and sexual abuse. American Journal of Psychiatry 147:887-92. [JJF]

Cioffi, D. \& Holloway, J. (1993) Delayed costs of suppressed pain. Journal of Personality and Social Psychology 64:274-82. [SN]

Cioffi, F. (1974/1998) Was Freud a liar? The Listener 91:172-74. (Reprinted in: F. Cioffi, Freud and the question of pseudoscience, pp. 199-204. Open Court, 1998.) [AE]

Claparède, E. (1911) Recognition et moiité. Archives de Psychologie Genève 11:79-80. [aMHE]

Clark, D. M., Ball, S. \& Pape, D. (1991) An experimental investigation of thought suppression. Behaviour Research and Therapy 29:253-57. [SN]

Cohen, N. J. \& Squire, R. L. (1980) Preserved learning and retention of patternanalyzing skill in amnesia: Dissociation of "knowing how" and "knowing that." Science 250:207-10. [aMHE]

Collette, F., van der Linden, M., Laureys, S., Delfiore, G., Degueldre, C. Luxen, A., \& Salmon, E. (2005) Exploring the unity and diversity of the neural substrates of executive functioning. Human Brain Mapping 25:409-23. [RES]

Conway, M. A., ed. (1997a) Recovered memories and false memories. Oxford University Press. [aMHE]

(1997b) Past and present: Recovered memories and false memories. In: Recovered memories and false memories, ed. M. A. Conway. Oxford University Press. [aMHE] 
2001) Repression revisited. Nature 410:319-20. [JFK]

Conway, M., Howell, A. \& Giannopoulos, C. (1991) Dysphoria and thought suppression. Cognitive Therapy and Research 15:153-66. [SN]

Corkin, S. (1968) Acquisition of motor skill after bilateral medial temporal lobe excision. Neuropsychologia 6:255-65. [aMHE]

Cramer, P. (1998) Coping and defense mechanisms: What's the difference? In: Defense mechanisms in contemporary personality research, ed. P. B. Cramer \& K. Davidson. Journal of Personality [Special Issue] 66:919-46. [aMHE]

(2000) Defense mechanisms in psychology today: Further processes for adaptation. American Psychologist 55:637-46. [aMHE, SB]

Crews, F. (1995) The memory wars: Freud's legacy in dispute. A New York Review Book. [aMHE, RJM]

(2006) Follies of the wise: Dissenting essays. Shoemaker Hoard. [RJM]

Crowder, R. G. (1976) Principles of learning and memory. Erlbaum. [arMHE]

Dalenberg, C. J. (1996) Accuracy, timing and circumstances of disclosure in therapy of recovered and continuous memories of abuse. Journal of Psychiatry and Law 24:229-76. [DHG]

Damasio, A. R. (1994) Descartes' Error: Emotion, reason, and the human brain. Avon Books. [aMHE]

Davidson, K. \& MacGregor, M. W. (1998) A critical appraisal of self-report defense mechanism measures. In: Defense mechanisms in contemporary personality research, ed. P. B. Cramer \& K. Davidson. Journal of Personality [Special Issue] 66:965-92. [aMHE]

Davis, D. (1990) Writing Freud. Faculty research talk at Haverford College. Available at: www.haverford.edu/psych/ddavis/f_writing.html [RGK]

Davis, P. J. \& Schwartz, G. E. (1987) Repression and the inaccessibility of affective memories. Journal of Personality and Social Psychology 52(1):155-62. [JMB]

de Decker, A., Hermans, D., Raes, F. \& Eelen, P. (2003) Autobiographical memory specificity and trauma in inpatient adolescents. Journal of Clinical Child and Adolescent Psychology 32:23-32. [DH]

Deese, J. (1959) On the prediction of occurrence of particular verbal intrusions in immediate recall. Journal of Experimental Psychology 58:17-22. [aMHE]

DePrince, A. P. (2005) Social cognition and revictimization risk. Journal of Trauma and Dissociation 6:125-41. [JJF]

DePrince, A. P. \& Freyd, J. J. (2004) Forgetting trauma stimuli. Psychological Science 15:488-92. [JJF]

Depue, B. E., Banich, M. T. \& Curran, T. (2006) Suppression of emotional and non-emotional content in memory: Effects of repetition on cognitive control. Psychological Science 17:441-47. [MCA]

Derakshan, N. \& Eysenck, M. W. (1997) Repression and repressors: Theoretical and experimental approaches. European Psychologist 2(3):235-46. [JMB]

Dickson, J. M. \& Bates, G. W. (2005) Influence of repression on autobiographical memories and expectations of the future. Australian Journal of Psychology 57:20-27. [DH]

Dillbeck, M. C. (1982) Meditation and flexibility of visual perception and verbal problem solving. Memory and Cognition 10(3):207-15. [AR]

Dollard, J. \& Miller, N. E. (1950) Personality and psychotherapy: An analysis in terms of learning, thinking, and culture. McGraw-Hill. [rMHE]

Duchenne de Bologne, G. B. (1862) The mechanism of human facial expression, trans. R. A. Cuthbertson. Cambridge University Press. [GAB]

Easterbrook, J. A. (1959) The effect of emotion on cue utilization and the organization of behavior. Psychological Review 66:183-201. [aMHE]

Ebbinghaus, H. (1885/1964) Memory: A contribution to experimental psychology, trans. H. A. Ruger \& C. E. Bussenius. Dover. [arMHE, MM]

Edwards, V. J., Fivush, R., Robert, F., Anda, R. F., Felitti, V. J. \& Nordenberg, D. F. (2001) Autobiographical memory disturbances in childhood abuse survivors. Journal of Aggression, Maltreatment, and Trauma 4:247-64. [JJF]

Eissler, K. R. (2001) Freud and the seduction theory: A brief love affair. International Universities Press. [AE]

Ekman, P. \& Friesen, W. V. (1982) Felt, false, and miserable smiles. Journal of Nonverbal Behavior 6:238-52. [GAB]

Elliott, D. M. (1997) Traumatic events: Prevalence and delayed recall in the general population. Journal of Consulting and Clinical Practice 65:811-20. [JJF]

Engle, R. W. (2000) What is working memory capacity? In: The nature of remembering: Essays in honor of Robert G. Crowder, ed. H. L. Roediger, J. S. Nairne, I. Neath \& A. M. Suprenant, pp. 297-314. American Psychological Association. [RES]

Epstein, W. (1972) Mechanisms of directed forgetting. In: The psychology of learning and motivation, vol. 6, ed. G. Bower, pp. 147-91. Academic Press. [aMHE, JFK]

Erdelyi, M. H. (1974) A new look at the New Look: Perceptual defense and vigilance. Psychological Review 81:1-25. [arMHE]

Erdelyi, M. H. (1985) Psychoanalysis: Freud's cognitive psychology. Freeman. [arMHE, JFK]

(1986) Experimental indeterminacies in the dissociation paradigm of subliminal perception. Behavioral and Brain Sciences 9:3-31. [aMHE]
(1990) Repression, reconstruction, and defense: History and integration of the psychoanalytic and experimental frameworks. In: Repression and dissociation: Implications for personality theory, psychopathology, and health, ed. J. L. Singer, pp. 1-31. Chicago University Press. [aMHE, JFK]

(1993) Repression: The mechanism and the defense. In: Handbook of mental control, ed. D. M. Wegner \& J. W. Pennebaker, pp. 126-48. Prentice Hall. [aMHE, JFK]

(1996) The recovery of unconscious memories: Hypermnesia and reminiscence. University of Chicago Press. [arMHE, JFK]

(1999) The unconscious, art, and psychoanalysis. In: Psychoanalysis and art at the millennium, ed. T. Ettinger. Psychoanalysis and Contemporary Thought [Special Issue] 22:609-26. [arMHE]

(2001a) Defense processes can be conscious or unconscious (Comment). American Psychologist 56:761-62. [aMHE]

(2001b) Studies in historicism: Archeological digs will not resolve the scientific questions of validity and reliability in free-association and interpretation. Psychological Inquiry 12:133-35. [aMHE]

(2004a) Comments on commentaries: Kihlstrom, Bachmann, Reingold, Snodgrass. Consciousness and Cognition 13:430-33. [arMHE]

(2004b) Subliminal perception and its cognates: Theory, indeterminacy, and time. Consciousness and Cognition 13:73-91. [arMHE]

Erdelyi, M. H. \& Appelbaum, A. (1973) Cognitive masking: The disruptive effect of an emotional stimulus upon the perception of contiguous neutral items. Bulletin of the Psychonomic Society 1:59-61. [aMHE]

Erdelyi, M. H. \& Blumenthal, D. (1973) Cognitive masking in rapid sequential processing: The effect of an emotional picture on preceding and succeeding pictures. Memory and Cognition 1:201-204. [aMHE]

Erdelyi, M. H., Finks, J. \& Feigin-Pfau, M. B. (1989) The effect of response bias on recall performance, with some observations on processing bias. Journal of Experimental Psychology: General 118:245-54. [rMHE]

Erdelyi, M. H. \& Goldberg, B. (1979) Let's not sweep repression under the rug: Toward a cognitive psychology of repression. In: Functional disorders of memory, ed. J. F. Kihlstrom \& F. J. Evans, pp. 355-402. Erlbaum. [arMHE, JFK]

Erdelyi, M. H. \& Kleinbard, J. (1978) Has Ebbinghaus decayed with time? The growth of recall (hypermnesia) over days. Journal of Experimental Psychology: Human Learning and Memory 4:275-89. [aMHE, SMS]

Erdelyi, M. H. \& Zizak, D. M. (2004) Beyond gizmo subliminality: In: Blurring the lines: The psychology of entertainment media, ed. L. J. Shrum, pp. 13-43. Erlbaum. [rMHE]

(in preparation) Beyond gizmo subliminality: The phenomena of the laboratory, the clinic, and the real world. [rMHE]

Eriksen, C. W. \& Pierce, J. (1968) Defense mechanisms. In: Handbook of personality theory and research, ed. E. Borgatta \& W. Lambert. Rand McNally. [aMHE]

Esterson, A. (1993) Seductive mirage: An exploration of the work of Sigmund Freud. Open Court. [AE, RJM]

(1998) Jeffrey Masson and Freud's seduction theory: A new fable based on old myths. History of the Human Sciences 11(1):1-21. Available at: http://human-nature.com/esterson/ [AE, RJM]

(2001) The mythologizing of psychoanalytic history: Deception and selfdeception in Freud's accounts of the seduction theory episode. History of Psychiatry 12:329-52. Available at: http://www.esterson.org/ Mythologizing_psychoanalytic_history.html [FC, AE, RJM]

(2002a) The myth of Freud's ostracism by the medical community in 1896-1905: Jeffrey Masson's assault on truth. History of Psychology 5:115-34. [RJM]

(2002b) Freud's seduction theory: A reply to Gleaves and Hernandez. History of Psychology 5:85-91. [RJM]

(2003) Freud's theories of repression and memory: A critique of Freud and false memory syndrome by Phil Mollon. The Scientific Review of Mental Health Practice (Fall-Winter) 2(2):147-62. Available at: http://www.srmhp.org/ 0202/review-01.html [AE, JFK]

(2006) A seductive story. Available at: http://www.butterfliesandwheels.com/ articleprint.php?num $=195 \quad[\mathrm{AE}]$

Everson, M. D. \& Boat, B. W. (1989) False allegations of sexual abuse by children and adolescents. Journal of the American Academy of Child and Adolescent Psychiatry 28:230-35. [GAB]

Fabrega, H., Jr. (1975) The need for an ethnomedical science. Science 189:969-75. [AR]

(1997) Evolution of sickness and healing. University of California Press. [AR] (2002) Origins of psychopathology: The phylogenetic and cultural basis of mental illness. Rutgers University Press. [AR]

Fan, J., Fossella, J., Sommer, T., Wu, Y. \& Posner, M. I. (2003) Mapping the genetic variation of executive attention onto brain activity. Proceedings of the National Academy of Science USA 100(12):7406-11. [AR]

Fechner, G. T. (1860/1966) Elements of psychophysics, trans. H. E. Adler. Holt, Rinehart \& Winston. [RGK]

Femina, D. D., Yeager, C. A. \& Lewis, D. O. (1990) Child abuse: Adolescent records vs. adult recall. Child Abuse and Neglect 14:227-31. [GAB] 
Festinger, L. A. (1957) A theory of cognitive dissonance. Stanford University Press. [JP]

Fisher, C. (1956) Dreams, images, and perception: A study of unconsciouspreconscious relationships. Journal of the American Psychoanalytic Association 4:380-445. [arMHE, MS]

(1988) Further observations on the Pötzl phenomenon: The effects of subliminal visual stimulation on dreams, images, and hallucinations. Psychoanalysis and Contemporary Thought 11:3-56. [rMHE]

Fivush, R. \& Haden, C. (2003) Autobiographical memory and the construction of a narrative self: Developmental and cultural perspectives. Erlbaum. [JP]

Fivush, R., M.-E. Pipe, M., Murachver, T. \& Reese, E. (1997) Events spoken and unspoken: Implications of language and memory development for the recovered memory debate. In: Recovered memories and false memories, ed. M. Conway, pp. 34-62. Oxford University Press. [JJF]

Foa, E. B., Molnar, C. \& Cashman, L. (1995a) Changes in rape narratives during exposure therapy for posttraumatic stress disorder. Journal of Traumatic Stress 8:675-90. [JP]

Foa, E. B. \& Riggs, D. S. (1993) Post-traumatic stress disorder and rape. In: American Psychiatric Press Review of Psychiatry, vol. 12, ed. J. Oldham, M. B. Riba \& A. Tasman, pp. 273-303. American Psychiatric Press. [JP]

Foa, E. B., Riggs, D. S., Massie, E. D. \& Yarczower, M. (1995b) The impact of fear activation and anger on the efficacy of exposure treatment for PTSD. Behavior Therapy 26:487-99. [GAB, rMHE]

Foa, E. B. \& Rothbaum, B. O. (1998) Treating the trauma of rape. Guilford. [GAB]

Fox, N. A., Henderson, H. A., Marshall, P. J., Nichols, K. E. \& Ghera, M. M. (2005) Behavioral inhibition: Linking biology and behaviour within a developmental framework. Annual Review of Psychology 56:235-62. [SB]

Foynes, M. M, Freyd, J. J. \& DePrince, A. P. (2006) Child Abuse, Betrayal, and Disclosure. Poster presented at the 22nd Annual Meeting of the International Society for Traumatic Stress Studies, Hollywood, CA, November 4-7, 2006. [JJF]

Frank, M., Ekman, P. \& Friesen, W. V. (1993) Behavioral markers and recognizability of the smile of enjoyment. Journal of Personality and Social Psychology 64:83-93. [GAB]

Freud, A. (1936/1937) The ego and the mechanisms of defense, trans. C. Baines. International Universities Press. [aMHE, JFK]

Freud, S. (1891/1953) On aphasia: A critical study, trans. E. Stengel. International Universities Press. [rMHE]

(1892-1893/1966) A case of successful treatment by hypnotism: With some remarks on the origin of hysterical symptoms through "counter-will." In: The standard edition of the complete psychological works of Sigmund Freud, vol. 1, ed. and trans. J. Strachey, pp. 117-28. Hogarth Press. [aMHE, MM, SN]

(1893/1966) Some points for a comparative study of organic and hysterical motor paralyses. In: The standard edition of the complete psychological works of Sigmund Freud, vol. 1, ed. \& trans. J. Strachey. Hogarth Press. [rMHE]

(1894/1962) The neuro-psychoses of defence, trans. J. Rickman. In: The standard edition of the complete psychological works of Sigmund Freud, vol. 3, ed. J. Strachey, pp. 41-61. Hogarth Press. [aMHE, MM]

(1896a/1962) Further remarks on the neuro psychoses of defence, trans. J. Rickman. In: The standard edition of the complete psychological works of Sigmund Freud, vol. 3, ed. J. Strachey, pp. 157-85. Hogarth Press. [aMHE, AE, DHG]

(1896b/1962) The aetiology of hysteria. In: The standard edition of the complete works of Sigmund Freud, vol. 3, ed. J. Strachey, pp.191-221. Hogarth Press. [RJM]

(1899/1962) Screen memories. In: The standard edition of the complete psychological works of Sigmund Freud, vol. 3, ed. J. Strachey, pp. 299-322. Hogarth Press. [AE, aMHE]

(1900/1987) Die Traumdeutung. Fischer Taschenbuch. [MS]

(1900/1913) The interpretation of dreams, trans. A. A. Brill. Allen and Unwin. [RGK]

(1900/1953) The interpretation of dreams, trans. J. Strachey. In: The standard edition of the complete psychological works of Sigmund Freud, vols. 4-5, ed. J. Strachey, pp. i-38 and pp. iii-627. Hogarth Press. [AE, arMHE]

(1901/1960) The psychopathology of everyday life, trans. A. Tyson. In: The standard edition of the complete psychological works of Sigmund Freud, vol. 6 , ed. J. Strachey, pp vii-310. Hogarth Press. [AE, aMHE]

(1905/1958) Jokes and their relation to the unconscious. In: The standard edition of the complete psychological works of Sigmund Freud, vol. 8, ed. and trans. J. Strachey, pp. 1-258. Hogarth Press. [arMHE, RL]

(1906a/1953) My views on the part played by sexuality in the aetiology of the neuroses. In: The standard edition of the complete psychological works of Sigmund Freud, vol. 7, ed. and trans. J. Strachey, pp. 269-79. Hogarth Press. [aMHE, AE]

(1906b/1959) Psycho-analysis and the establishment of the facts in legal proceedings, trans. E. B. M. Herford. In: The standard edition of the complete psychological works of Sigmund Freud, vol. 9, ed. J. Strachey, pp. 99-114. Hogarth Press. [aMHE]
(1909/1955) Notes upon a case of obsessional neurosis, trans. A. Strachey \& J. Strachey. In: The standard edition of the complete psychological works of Sigmund Freud, vol. 10, ed. J. Strachey, pp. 151-249. Hogarth Press. [aMHE]

(1910a/1957) Five lectures on Psycho-analysis. In: The standard edition of the complete psychological works of Sigmund Freud, vol. 11, ed. J. Strachey. Hogarth Press. [SB]

(1910b/1957) "Wild" psycho-analysis, trans. J. Riviere. In: The standard edition of the complete psychological works of Sigmund Freud, vol. 11, ed. J. Strachey,. Hogarth Press. [SB]

(1911/1958) Psycho-analytic notes on an autobiographical account of a case of paranoia (dementia paranoides), trans. A. Strachey \& J. Strachey. In: The standard edition of the complete psychological works of Sigmund Freud, vol. 12, ed. J. Strachey, pp. 1-82. Hogarth Press. [aMHE]

(1912/1958) A note on the unconscious in psycho-analysis. In: The standard edition of the complete psychological works of Sigmund Freud, vol. 12, ed. J. Strachey. Hogarth Press. [SB]

(1914a/1958) Remembering, repeating and working through, trans. J. Riviere. In: The standard edition of the complete psychological works of Sigmund Freud, vol. 12, ed. J. Strachey, pp. 145-56. Hogarth Press. [aMHE, GS]

(1914b/1958) The history of the psycho-analytic movement, trans. J. Riviere. In: The standard edition of the complete psychological works of Sigmund Freud, vol. 14, ed. J. Strachey, pp. 3-66. Hogarth Press. [arMHE]

(1915a/1957/1963) Repression, trans. C. M. Baines \& J. Strachey. In: The standard edition of the complete psychological works of Sigmund Freud, vol. 14, ed. J. Strachey, pp. 146-58. Hogarth Press, 1957. [SB, AE, aMHE, JFK, RL]. Also in: Freud: General psychological theory, ed. P. Rieff, trans. C. M. Baines, pp. 104-115. Collier, 1963. [MCA, JMB, aMHE]

(1915b/1957/1963) The unconscious, trans. C. M. Baines \& J. Strachey. In: The standard edition of the complete psychological works of Sigmund Freud, vol. 14, ed. J. Strachey, pp. 159-204. Hogarth Press, 1957. Also in: Freud: General psychological theory, trans. C. M. Baines, ed. P. Rieff, pp. 116-150. Collier, 1963. [aMHE, JFK, RL]

(1916/1922) A general introduction to psychoanalysis, trans. J. Riviere. Allen and Unwin. [RGK]

(1916-1917/1961) Introductory lectures on psychoanalysis. In: The standard edition of the complete psychological works of Sigmund Freud, vols. 15-16, ed. J. Strachey. Hogarth Press. [RL]

(1917/1961/1963) A general introduction to psychoanalysis, trans. J. Riviere. Liveright, 1963. (Also published as: Introductory lectures on psychoanalysis. In: The standard edition of the complete psychological works of Sigmund Freud, vols. 15-16, ed. J. Strachey, p. iii-239; p. iii-496. Hogarth Press, 1961 and 1963). [arMHE]

(1920/1955) Beyond the pleasure principle. In: The standard edition of the complete psychological works of Sigmund Freud, vol. 18, ed. and trans. J. Strachey, pp. 1-64. Hogarth Press. [aMHE]

(1923/1961) The ego and the id, trans. J. Riviere. In: The standard edition of the complete psychological works of Sigmund Freud, vol. 19, ed. J. Strachey, pp. 1-66. Hogarth Press. [aMHE, SB, RL]

(1926a/1959) Inhibition, symptoms and anxiety, trans. A. Strachey \& J. Strachey. In: The standard edition of the complete psychological works of Sigmund Freud, vol. 20, ed. J. Strachey, pp. 75-175. Hogarth Press. [aMHE, SB, RL] (1926b/1959) Psycho-analysis, trans. A. Strachey \& J. Strachey. In: The standard edition of the complete psychological works of Sigmund Freud, vol. 20, ed. J. Strachey. Hogarth Press. [RL]

(1933/1964) New introductory lectures on psycho-analysis. In: The standard edition of the complete psychological works of Sigmund Freud, vol. 22, trans. and ed. J. Strachey, pp. 1-158. Hogarth Press. [aMHE, AE, RL]

(1936/1960) A disturbance of memory on the Acropolis. In: The standard edition of the complete psychological works of Sigmund Freud, vol. 22, trans. and ed. J. Strachey. Hogarth Press. [SB]

(1937/1964) Constructions in analysis. In: The standard edition of the complete psychological works of Sigmund Freud, vol. 23, ed. and trans. J. Strachey, pp. 255-69. Hogarth Press. [aMHE, RES]

(1938/1964) Some elementary lessons in psycho-analysis. In: The standard edition of the complete psychological works of Sigmund Freud, vol. 23, trans. and ed. J. Strachey, pp. 279-86. Hogarth Press. [aMHE]

(1939/1964) Moses and monotheism: Three essays. The standard edition of the complete psychological works of Sigmund Freud, vol. 23, trans. and ed. J. Strachey. Hogarth Press. [SB]

(1940/1964) An outline of psycho-analysis. In: The standard edition of the complete psychological works of Sigmund Freud, vol. 23, trans. and ed. J. Strachey, pp. 139-207. Hogarth Press. [aMHE]

Freyd, J. J. (1996) Betrayal trauma: The logic of forgetting childhood abuse. Harvard University Press. [aMHE]

(1983) Shareability: The social psychology of epistemology. Cognitive Science 7:191-210. [JJF]

(1990) Natural selection or shareability? Behavioral and Brain Sciences 13:732-34. [JJF] 
(1994) Betrayal-trauma: Traumatic amnesia as an adaptive response to childhood abuse. Ethics and Behavior 4:307-29. [JJF]

(1996) Betrayal trauma: The logic of forgetting childhood abuse. Harvard University Press. [JJF]

(1999) Blind to betrayal: New perspectives on memory for trauma. The Harvard Mental Health Letter 15(12):4-6. [JJF]

(2001) Memory and dimensions of trauma: Terror may be "all-too-well remembered" and betrayal buried. In: Critical issues in child sexual abuse: Historical, legal, and psychological perspectives, ed. J. R. Conte, 139-73. Sage. [JJF]

(2003) Memory for abuse: What can we learn from a prosecution sample? Journal of Child Sexual Abuse 12:97-103. [JJF]

Freyd, J. J., DePrince, A. P. \& Zurbriggen, E. L. (2001) Self-reported memory for abuse depends upon victim-perpetrator relationship. Journal of Trauma and Dissociation 2:5-17. [JJF]

Freyd, J. J. \& Gleaves, D. H. (1996) Remembering words not presented in lists: Relevance to the current recovered/false memory controversy. Journal of Experimental Psychology: Learning, Memory, and Cognition 22:811-13. [DHG]

Freyd, J. I., Putnam, F. W., Lyon, T. D., Becker-Blease, K. A., Cheit, R. E., Siegel, N. B. \& Pezdek, K. (2005) The science of child sexual abuse. Science 308:501. [JJF]

Friedman, N. P. \& Miyake, A. (2004) The relations among inhibition and interference control functions: A latent-variable analysis. Journal of Experimental Psychology: General 133:101-35. [RES]

Garro, L. C. (2001) The remembered past in a culturally meaningful life: Remembering as cultural, social, and cognitive process. In: The psychology of cultural experience, ed. C. C. Moore \& H. F. Matthews, pp. 105-47. Cambridge University Press. [AR]

Gazzaniga, M. S. (1985) The social brain: Discovering the networks of the mind. BasicBooks. [aMHE]

Geiselman, R. E., Bjork, R. A. \& Fishman, D. L. (1983) Disrupted retrieval in directed forgetting: A link with posthypnotic amnesia. Journal of Experimental Psychology: General 112:58-72. [aMHE]

Geraerts, E., Smeets, E., Jelicic, M., Merckelbach, H. \& van Heerden, J. (2006) Retrieval inhibition of trauma-related words in women reporting repressed and recovered memories of childhood sexual abuse. Behaviour Research and Therapy 44:1129-36. [RJM]

Gilbert, D. T. (1991) How mental systems believe. American Psychologist 46:107-19. [aMHE]

Gilbertson, M. W., Shenton, M. E., Ciszewiski, A., Kasai, K., Lasko, N. B., Orr, S. O. \& Pitman, R. K. (2002) Smaller hippocampal volume predicts pathologic vulnerability to psychological trauma. Nature Neuroscience 5:1242-47. [rMHE]

Gildston, P. (1998) Nonreplicating white bears and other irrepressible cautions. Unpublished doctoral dissertation. City University of New York. [aMHE, SN]

Gleaves, D. H. (1996) The evidence for "repression": An examination of Holmes (1990) and the implications for the recovered memory controversy. Journal of Child Sexual Abuse 5:1-19. [DHG]

Gleaves, D. H. \& Hernandez, E. (1999) Recent reformulations of Freud's alleged discoveries of childhood sexual abuse: Historical/scientific clarification or a continued assault on truth? History of Psychology 2:1-31. [DHG]

Gleaves, D. H., Smith, S. M., Butler, L. D. \& Spiegel, D. (2004) False and recovered memories in the laboratory and clinic: A review of experimental and clinical evidence. Clinical Psychology: Science and Practice 11:3-28. [DHG, JFK]

Glucksberg, S. \& King, L. J. (1967) Motivated forgetting mediated by implicit verbal chaining: A laboratory analog of repression. Science 27:517-19. [rMHE]

Goddard, L., Dritschel, B. \& Burton, A. (1996) Role of autobiographical memory in social problem solving and depression. Journal of Abnormal Psychology 105:609-16. [DH]

Green, J. D., Pinter, B. T. \& Sedikides, C. (2005) Mnemic neglect and self-threat: Trait modifiability moderates self-protection. European Journal of Social Psychology 35:225-35. [CS]

Green, J. D. \& Sedikides, C. (2004) Retrieval selectivity in the processing of selfreferent information: Testing the boundaries of self-protection. Self and Identity 3:69-80. [CS]

Green, J. D., Sedikides, C. \& Gregg, A. P. (2006) Forgotten but not gone: The recall and recognition of self-threatening memories. Unpublished manuscript. Virginia Commonwealth University. [CS]

Greenwald, A. G. (1980) The totalitarian ego: Fabrication and revision of personality history. American Psychologist 5:603-18. [aMHE]

Grinker, R. R. \& Spiegel, J. P. (1945) War neuroses. Blakiston. [JJF]

Gross, J., Hayne, H. \& Poole, A. (2006) The use of drawing in interviews with children: A potential pitfall. In: Focus on child psychology research, ed. J. R. Marrow, pp. 119-44 Nova Publishers. [HH]

Haber, R. N. \& Erdelyi, M. H. (1967) Emergence and recovery of initially unavailable perceptual material. Journal of Verbal Learning and Verbal Behavior
References/Erdelyi: The unified theory of repression

6:618-28. (Also in: Contemporary theory and research in visual perception, ed. R. N. Haber. Holt, Rinehart \& Winston, 1986). [rMHE]

Halbwachs, M. (1951/1992) On collective memory, trans. L. A. Coser. University of Chicago Press. (Original work published 1951). [JP]

Hartmann, E. (1998) Dreams and nightmares: The new theory on the origin and meaning of dreams. Plenum Press. [RGK]

Hartmann, E., Kunzendorf, R. G., Baddour, A., Chapwick, M., Eddins, M., Krueger, C., Latraverse, T. \& Shannon, R. (2002-2003) Emotion makes daydreams more dreamlike, more symbolic. Imagination, Cognition, and Personality 22:255-74. [RGK]

Harvey, A. G. \& Bryant, R. A. (1998) The effect of attempted thought suppression in acute stress disorder. Behavior Research and Therapy 36:583-90. [SN]

Harvey, M. R. \& Herman, J. L. (1994) Amnesia, partial amnesia and delayed recall among adult survivors of childhood trauma. Consciousness and Cognition 3:295-306. [MCA]

Henderson, D., Hargreaves, I., Gregory, S. \& Williams, J. M. G. (2002) Autobiographical memory and emotion in a non-clinical sample of women with and without a reported history of childhood sexual abuse. British Journal of Clinical Psychology 41:129-41. [DH]

Herbart, J. F. (1824-1825) Psychologie als Wissenschaft neu gegründet auf Erfahrung, Metaphysik und Mathematik, vols. 1 and 2. Unzer. [aMHE]

Herman, J. H. (1992) Trauma and recovery. BasicBooks. [RJM]

Herman, J. H. \& Schatzow, E. (1987) Recovery and verification of memories of childhood sexual trauma. Psychoanalytic Psychology 4:1-14. [RJM]

Hermans, D., Defranc, A., Raes, F., Williams, J. M. G. \& Eelen, P. (2005) Reduced autobiographical memory specificity as an avoidant coping style. British Journal of Clinical Psychology 44:583-89. [DH]

Hermans, D., van den Broeck, K., Belis, G., Raes, F., Pieters, G. \& Eelen, P. (2004) Trauma and autobiographical memory specificity in depressed inpatients. Behaviour Research and Therapy 42:775-89. [DH]

Herrmann, D. J., Buschke, H. \& Gall, M. B. (1987) Improving retrieval. Applied Cognitive Psychology 1:27-33. [aMHE]

Het, S., Ramlow, G. \& Wolf, O. T. (2005) A meta-analytic review of the effects of acute cortisol administration on human memory. Psychoneuroendocrinology 30:771-84. [EF]

Holender, D. (1986) Semantic activation without conscious identification in dichotic listening, parafoveal vision, and visual masking: A survey and appraisal. Behavioral and Brain Sciences 9:1-66. [aMHE]

Holmes, D. (1990) The evidence for repression: An examination of sixty years of research. In: Repression and dissociation: Implications for personality theory, psychopathology, and health, ed. J. Singer, pp. 85-102. University of Chicago Press. [FC]

Holmes, D. S. (1974) Investigations of repression: Differentiated recall of material experimentally or naturally associated with ego threat. Psychological Bulletin 81:632-53. [arMHE, GAB]

(1990) The evidence for repression: An examination of sixty years of research. In: Repression and dissociation: Defense mechanisms and personality styles, ed. J. L. Singer. Chicago University Press. [aMHE]

(1990) The evidence for repression: An examination of sixty years of research. In: Repression and dissociation: Implications for personality theory, psychopathology, and health, ed. J. L. Singer, pp. 85-102. University of Chicago Press. [RGK]

Holtgraves, T. \& Hall, R. (1995) Repressors: What do they repress and how do they repress it. Journal of Research in Personality 29(3):306-17. [JMB]

Horowitz, M. J. (1986) Stress response syndrome, 2nd edition. Aronson. [aMHE]

Ionescu, M. D. \& Erdelyi, M. H. (1992) The direct recovery of subliminal stimuli. In: Perception without awareness, ed. R. F. Bornstein \& T. S. Pittman. Guilford Press. [aMHE]

Israëls, H. \& Schatzman, M. (1993) The seduction theory. History of Psychiatry 4:23-59. [FC, AE]

Jack, D. C. (1999) Silencing the self: Inner dialogue and outer realities. In: The interactional nature of depression, ed. T. Joiner \& J. C. Coyne, pp. 221-46. American Psychological Association Press. [GS]

Jacoby, L. L., Kelley, C. M., Brown, J. \& Jasechko (1989a) Becoming famous overnight: Limits on the ability to avoid unconscious influence of the past. Journal of Personality and Social Psychology 56:326-38. [aMHE]

Jacoby, L. L., Kelley, C. M. \& Dywan, J. (1989b) Memory attributions. In: Varieties of memory and consciousness: Essays in Honour of Endel Tulving, ed. H. L. Roediger \& F. I. M. Craik, pp. 391-422. Erlbaum. [aMHE]

Jacobs, W. J. \& Nadel, L. (1998) Neurobiology of reconstructed memory. Psychology: Public Policy and Law 4:1110-34. [DHG]

Jacoby, L. L. \& Witherspoon, D. (1982) Remembering without awareness. Canadian Journal of Psychology 32:300-24. [aMHE]

Janet, P. (1889) L'Automatisme psychologique. Alcan. [arMHE] (1907) The major symptoms of hysteria. Macmillan. [RGK]

(1910) Chapter four. In: Subconscious phenomena, ed. H. Münsterberg, T. Ribot, P. Janet, J. Mastrow, B. Hart \& M. Prince, pp. 53-70. Gorham Press. [RGK]

Jenkins, J. J. \& Dallenbach, K. M. (1924) Oblivescence during sleep and waking. American Journal of Psychology 35:605-12. [aMHE] 
Joorman, J., Hertel, P. T., Brozovich, F. \& Gotlib, I. H. (2005) Remembering the good, forgetting the bad: Intentional forgetting of emotional material in depression. Journal of Abnormal Psychology 114:640-48. [MCA]

Joslyn, S. L. \& Oakes, M. A. (2005) Directed forgetting of autobiographical events. Memory and Cognition 33:577-87. [MCA]

Juni, S. (1997) Conceptualizing defense mechanisms from drive theory and object relations perspectives. The American Journal of Psychoanalysis 57:149-66. [SB]

Kaufman, J., Yang, B., Douglas-Palumberi, H., Houshyar, S., Lipschitz, D., Krystal, J. \& Gelernter, J. (2004) Social supports and serotonin transporter gene moderate depression in maltreated children. Proceedings of the National Academy of Sciences USA 101(49):17316-21. [JP]

Kawaguchi, J., Hotta, C. \& Takei, S. (2006) Implicit memory for intentionally suppressed information. Poster presented at the 18th Annual Meeting of the Association for Psychological Science, New York, NY, May 25-28. [MCA]

Kelly, A. E. \& Kahn, J. H. (1994) Effects of suppression of personal intrusive thoughts. Journal of Personality and Social Psychology 66:998-1006. [SN]

Keltner, D. \& Buswell, B. N. (1997) Embarrassment: Its distinct form and appeasement functions. Psychological Bulletin 122:250-70. [GAB]

Kihlstrom, J. F. (1997) Suffering from reminiscences: Exhumed memory, implicit memory, and the return of the repressed. In: Recovered memories and false memories, ed. M. A. Conway, pp. 100-117. Oxford University Press. [aMHE]

(2002) No need for repression. Trends in Cognitive Sciences 6:502. [JFK]

(2004) An unbalanced balancing act: Blocked, recovered, and false memories in the laboratory and the clinic. Clinical Psychology: Science and Practice 11:34-41. [JFK]

(2006) Trauma and memory revisited. In: Memory and Emotions: Interdisciplinary Perspectives, ed. B. Uttl, N. Ohta \& A. L. Siegenthaler, pp. 259-91. Blackwell. [aMHE, JFK]

Kihlstrom, J. F. \& Barnhardt, T. M. (1993) The self-regulation of memory: For better and for worse, with and without hypnosis. In: Handbook of mental control, ed. D. M. Wegner \& J. W. Pennebaker, pp. 88-125. Prentice Hall. [JFK]

Kihlstrom, J. F. \& Cantor, N. (1984) Mental representations of the self. In: Advances in experimental social psychology, vol. 17, ed. L. Berkowitz, pp. 1-47. Academic Press. [RGK]

Kluft, R. P. (1987a) An update on multiple personality disorder. Hospital and Community Psychiatry 38:363-73. [RGK]

(1987b) First-rank symptoms as a diagnostic clue to multiple personality disorder. American Journal of Psychiatry 144:293-98. [RGK]

Korfine, L. \& Hooley, J. M. (2000) Directed forgetting of emotional material in borderline personality disorder. Journal of Abnormal Psychology 109:214-21. [RJM]

Kris, E. (1956/1975) The personal myth: A problem in psychoanalytic technique. In: The selected papers of Ernst Kris, ed. L. M. Newman. Yale University Press. (Original paper published in 1956). [aMHE]

Kuhlmann, S., Piel, M. \& Wolf, O. T. (2005) Impaired memory retrieval after psychosocial stress in healthy young men. Journal of Neuroscience $25: 2977-82 . \quad[\mathrm{EF}]$

Kunzendorf, R. G. (1989-1990) Posthypnotic amnesia: Dissociation of self-concept or self-consciousness? Imagination, Cognition, and Personality 9:321-34. [RGK]

(2000) Individual differences in self-conscious source monitoring: Theoretical, experimental, and clinical considerations. In: Individual differences in conscious experience, ed. R. G. Kunzendorf \& B. Wallace, pp. 375-90. John Benjamins. [RGK]

Kunzendorf, R. G. \& Boisvert, P. (1996) Presence vs. absence of a "hidden observer" during total deafness: The hypnotic illusion of subconsciousness vs. the imaginal attenuation of brainstem auditory evoked potentials. In: Hypnosis and imagination, ed. R. G. Kunzendorf, N. P. Spanos \& B. Wallace, pp. 223-34. Baywood. [RGK]

Kunzendorf, R. G., Hartmann, E., Thomas, L., Berensen, L. (1999-2000) Emotionally directing visual sensations: I. Generating images that contextualize emotion and become "symbolic." Imagination, Cognition, and Personality 19:269-78. [RGK]

Kunzendorf, R. G., Hulihan, D. M., Simpson, W., Pritykina, N. \& Williams, K. (1997-1998) Is absorption a diathesis for dissociation in sexually and physically abused patients? Imagination, Cognition, and Personality 17:277-82. [RGK]

Kunzendorf, R. G. \& Karpen, J. (1996-1997) Dissociative experiences and realitytesting deficits in college students. Imagination, Cognition, and Personality 16:227-38. [RGK]

Kunzendorf, R. G. \& Maurer, J. (1988-1989) Hypnotic attenuation of the "boundaries" between emotional, visual, and auditory sensations. Imagination, Cognition, and Personality 8:225-34. [RGK]

Kunzendorf, R. G. \& Moran, C. (1993-1994) Repression: Active censorship of stressful memories vs. source amnesia for self-consciously dissociated memories. Imagination, Cognition, and Personality 13:291-302. [RGK]
Kunzendorf, R. G. \& Paroskie, J. (2000-2001) Emotionally directing visual sensations: II. Lowering the threshold for tinted percepts. Imagination, Cognition, and Personality 20:179-87. [RGK]

Kuyken, W. \& Brewin, C. R. (1995) Autobiographical memory functioning in depression and reports of early abuse. Journal of Abnormal Psychology 104:585-91. [DH]

Lavy, E. H. \& van den Hout, M. A. (1990) Thought suppression induces intrusions. Behavioural Psychotherapy 18:251-58. [SN]

LeDoux, J. E. (1996) The emotional brain: The mysterious underpinnings of emotional life. Simon \& Schuster. [aMHE]

Levy, B. J. \& Anderson, M. C. (2002) Inhibitory processes and the control of memory retrieval. Trends in Cognitive Sciences 6:299-305. [MCA, aMHE, JFK]

Loftus, E. F. (1979) Eyewitness testimony. Harvard University Press. [aMHE] (1993) The reality of repressed memories. American Psychologist 48:518-37. [MCA]

Loftus, E. F. \& Bernstein, D. M. (2005) Rich false memories. In: Experimental cognitive psychology and its applications, ed. A. F. Healy, pp. 103-13. APA Press. [HH]

Loftus, E. F. \& Burns, T. E. (1982) Mental shock can produce retrograde amnesia. Memory and Cognition 10:318-23. [aMHE]

Loftus, E. F., Garry, M. \& Feldman, J. (1994) Forgetting sexual trauma: What does it mean when 38\% forget? Journal of Consulting and Clinical Psychology 62:1177-81. [GAB]

Loftus, E. F. \& Guyer, M. J. (2002a) Who abused Jane Doe? The hazards of the single case history: Part I. Skeptical Enquirer 26:24-32. [HH]

(2002b) Who abused Jane Doe? Part II. Skeptical Inquirer 26:37-40, 44. [HH]

Loftus, E. F. \& Ketcham, K. (1994) The myth of repressed memory: False memories and allegations of sexual abuse. St. Martin's Press. [MCA, arMHE]

Loftus, E. F. \& Palmer, J. C. (1974) Reconstruction of automobile destruction: An example of the interaction between language and memory. Journal of Verbal Learning and Verbal Behavior 13:585-89. [aMHE]

Loftus, E. F. \& Pickrell, J. E. (1995) The formation of false memories. Psychiatric Annals 25:720-25. [MCA]

Loftus, E. F., Miller, D. G. \& Burns, H. J. (1978) Semantic integration of verbal information into a visual memory. Journal of Experimental Psychology: Human Learning and Memory 4:19-31. [MCA, aMHE]

Luborsky, L., Crits-Christoph, P. \& Alexander, K. J. (1990) Repressive style and relational patterns - Three samples inspected. In: Repression and dissociation: Implications for personality, theory, psychopathology, and health, ed. J. L. Singer, pp. 275-98. University of Chicago Press. [rMHE]

Lynn, S. J. \& Pintar J. (1997) A social narrative model of dissociative identity disorder. Australian Journal of Clinical and Experimental Hypnosis 25:17. [JP]

Lynn, S. J., Pintar, J. \& Rhue, J. (1997) Fantasy proneness, dissociation, and narrative construction. In: Broken images, broken selves: Dissociative narratives in clinical practice, ed. S. Powers \& S. Krippner, pp. 274-304. Brunner/Mazel. [JP]

Lynn, S. J., Pintar, J., Sandberg, D., Fite, D., Ecklund, K. \& Stafford, J. (2003) Towards a social narrative model of revictimization. In: From child sexual assault to adult sexual risk: Trauma, revictimization, and intervention, ed. L. Koenig, A. O’Leary, L. Doll \& W. Pequenat. American Psychological Association. [JP]

MacLeod, C. M., Dodd, M. D., Sheard, E. D., Wilson, D. E. \& Bibi, U. (2003) In opposition to inhibition. The Psychology of Learning and Motivation 43:163-214. [RES]

Macmillan, M. (1997) Freud evaluated: The completed arc. MIT Press. [JFK, RJM, MM]

(2001) Limitations to free-associations and interpretation. Psychological Inquiry 12:113-75. [aMHE]

MacLeod, M. D. \& Macrae, C. N. (2001) Gone but not forgotten: The transient nature of retrieval-induced forgetting. Psychological Science 12:148-52. [MCA]

Macmillan, N. A. (1986) The psychophysics of subliminal perception. Behavioral and Brain Sciences 9:38-39. [aMHE]

Macmillan, N. A. \& Creelman, C. D. (2004) Detection theory: A user's guide. Erlbaum. [aMHE]

Macrae, C. N., Bodenhausen, G. V., Milne, A. B. \& Ford, R. L. (1997) On regulation of recollection: Intentional forgetting of stereotypical memories. Journal of Personality and Social Psychology 72:709-19. [SN]

Macrae, C. N., Bodenhausen, G. V., Milne, A. B. \& Jetten, J. (1994) Out of mind but back in sight: Stereotypes on the rebound. Journal of Personality and Social Psychology 67:808-17. [SN]

Madison, P. (1961) Freud's concept of repression and defense: Its theoretical and observational language. University of Minnesota Press. [SB]

Maratos, E. J., Dolan, R. J., Morris, J. S., Henson, R. N. A. \& Rugg, M. D. (2001) Neural activity associated with episodic memory for emotional context. Neuropsychologia 39:910-20. [NM]

Marcel, A. J. (1983a) Conscious and unconscious perception: An approach to the relation between phenomenal experience and cognitive processes. Cognitive Psychology 15:197-237. [aMHE] 
(1983b) Conscious and unconscious perception: Experiments on visual masking and word recognition. Cognitive Psychology 15:197-237. [aMHE]

Markowitsch, H. J. (1999) Functional neuroimaging correlates of functional amnesia. Memory 7:561-83. [rMHE]

Markus, H. \& Nurius, P. (1986) Possible selves. American Psychologist 41:954-69. [aMHE]

Mayr, U., Awh, E. \& Keele, S. W. (2005) Developing individuality in the human brain: A tribute to Michael I. Posner. American Psychological Association. [AR]

Maze, J. R. (1983) The meaning of behaviour. Allen and Unwin. [SB]

Maze, J. R. \& Henry, R. M. (1996) Problems in the concept of repression and proposals for their resolution. International Journal of Psycho-Analysis $77: 1085-1100 . \quad[\mathrm{SB}]$

Mazzoni, G. \& Loftus, E. F. (1998) Dreaming, believing, and remembering. In: Believing-in imaginings: The narrative construction of reality, ed. J. de Rivera \& T. R. Sarbin, pp. 145-56. American Psychological Association Press. [MCA]

Mazzoni, G. A. L., Loftus, E. F. \& Kirsch, I. (2001) Changing beliefs about implausible autobiographical events: A little plausibility goes a long way. Journal of Experimental Psychology: Applied 7:51-59. [MCA]

McAdams, D. P. (1998) The role of defense in the life story. In: Defense mechanisms in contemporary personality research, ed. P. B. Cramer \& K. Davidson Journal of Personality [Special Issue] 66:1125-46. [aMHE]

McGeoch, J. A. (1932) Forgetting and the law of disuse. Psychological Review 39:352-70. [aMHE]

McNally, R. J. (2003) Remembering trauma. Belknap Press/Harvard University Press. [GAB, arMHE, JFK, JP]

(2005) Directed forgetting tasks in clinical research. In: Cognitive methods and their application to clinical research, ed. A. Wenzel \& D. C. Rubin, pp. 197-212. American Psychological Association Press. [RJM]

McNally, R. J., Clancy, S. A., Barrett, H. M. \& Parker, H. A. (2004) Inhibiting retrieval of trauma cues in adults reporting histories of childhood sexual abuse. Cognition and Emotion 18:479-93. [RJM]

McNally, R. J., Clancy, S. A. \& Schacter, D. L. (2001) Directed forgetting of trauma cues in adults reporting repressed or recovered memories of childhood sexual abuse. Journal of Abnormal Psychology 110:151-56. [RJM]

McNally, R. J., Metzger, L. J., Lasko, N. B., Clancy, S. A. \& Pitman, R. K. (1998) Directed forgetting of trauma cues in adult survivors of childhood sexual abuse with and without posttraumatic stress disorder. Journal of Abnormal Psychology 107:596-601. [RJM]

McNally, R. J., Otto, M. W., Yap, L., Pollack, M. W. \& Hornig, C. D. (1999) Is panic disorder linked to cognitive avoidance of threatening information? Journal of Anxiety Disorders 13:335-48. [RJM]

McNally, R. J. \& Ricciardi, J. N. (1996) Suppression of negative and neutral thoughts. Behavioural and Cognitive Psychotherapy 24:17-25. [SN]

McNally, R. J., Ristuccia, C. S. \& Perlman, C. A. (2005) Forgetting of trauma cues in adults reporting continuous or recovered memories of childhood sexual abuse. Psychological Science 16:336-40. [RJM]

Medford, N., Phillips, M. L., Brierley, B., Brammer, M., Bullmore, E. T. \& David, A. S. (2005a) Emotional memory: Separating content and context. Psychiatry Research Neuroimaging 138:247-58. [NM]

Medford, N., Sierra, M., Baker, D. \& David, A. S. (2005b) Understanding and treating depersonalisation disorder. Advances in Psychiatric Treatment $11: 92-100 . \quad[\mathrm{NM}]$

Mendolia, M. (2002) An index of self-regulation of emotion and the study of repression in social contexts that threaten self-concept. Emotion 2:215-32. [DH]

Merikle, P. M. \& Reingold, E. M. (1991) Comparing direct (explicit) and indirect (implicit) measures to study unconscious memory. Journal of Experimental Psychology: Learning, Memory, and Cognition 17:224-33. [aMHE]

Michell, J. (1988) Maze's direct realism and the character of cognition. Australian Journal of Psychology 40:227-49. [SB]

Migueles, M. \& Garcia-Bajos, E. (submitted) Partial retrieval and induced forgetting in eyewitness memory. [MCA]

Mogg, K. \& Bradley, B. P. (1998) A cognitive-motivational analysis of anxiety. Behavioral Research and Therapy 36:809-48. [aMHE]

Moore, E. (2004) Suppression and rebound: The inhibited fairy-tale. Unpublished master's thesis. Brooklyn College, City University of New York. [aMHE]

Moulds, M. L. \& Bryant, R. A. (2002) Directed forgetting in acute stress disorder Journal of Abnormal Psychology 111:175-79. [RJM]

(2005) An investigation of retrieval inhibition in acute stress disorder. Journal of Traumatic Stress 18:233-36. [RJM]

Myers, L. B. \& Derakshan, N. (2004) To forget or not to forget: What do repressors forget and when do they forget? Cognition and Emotion 18:495-511. [EF]

Neisser, U., ed. (1982) Memory observed: Remembering in natural contexts. W. H. Freeman. [aMHE]

Nigg, J. T. (2000) On inhibition/disinhibition in developmental psychopathology: Views from a cognitive and personality psychology and a working inhibition taxonomy. Psychological Bulletin 126:220-46. [SB]
Nishioka, K. \& Kozbelt, A. (in preparation) Humor comprehension, humor production, and insight. [aMHE]

Nolen-Hoeksema, S. (1990) Sex differences in unipolar depression: Evidence and theory. Psychological Bulletin, 101:259-82. [GS]

Obholzer, K. (1982) The Wolf-Man sixty years later: Conversations with Freud's controversial patient, trans. M. Shaw. Routlege. $[\mathrm{FC}]$

Ochsner, K. N., Ray, R. D., Cooper, J. C., Robertson, E. R., Chopra, S., Gabrieli, J. D. \& Gross, J. J. (2004) For better or for worse: Neural systems supporting the cognitive down- and up-regulation of negative emotion. Neuroimage 23:483-99. [EF, NM]

Ohira, H., Nomura, M., Ichikawa, N., Isowa, T., Iidaka, T., Sato, A., Fukuyama, S., Nakajima, T. \& Yamada, J. (2006) Association of neural and physiological responses during voluntary emotion suppression. Neuroimage 29:721-33. [NM]

Öhman, A. \& Soares, J. J. (1994) "Unconscious anxiety”: Phobic responses to masked stimuli. Journal of Abnormal Psychology 103:231-40. [MS]

Olio, K. (1994) Truth in memory. American Psychologist 49:442-43. [MCA]

Parkin, A. J. (1987) Memory and amnesia: An introduction. Blackwell. [aMHE]

Pasnau, R. (1997) Theories of cognition in the later Middle Ages. Cambridge University Press. [SB]

Paulhus, D. L. \& John, O. P. (1998) Egoistic and moralistic biases in self perception: The interplay of self-deceptive styles with basic traits and motives. In: Defense mechanisms in contemporary personality research, ed. P. B. Cramer \& K. Davidson. Journal of Personality [Special Issue] 66:1025-60. [aMHE]

Pavlov, I. P. (1927) Conditioned reflexes: An investigation of the physiological activation of the cerebral cortex. Oxford University Press. [aMHE]

Payne, D. G. (1987) Hypermnesia and reminiscence in recall: A historical and empirical review. Psychological Bulletin 101:5-27. [aMHE]

Perloff, L. S. \& Fetzer, B. K. (1986) Self-other judgments and perceived vulnerability to victimization. Journal of Personality and Social Psychology 50:502-10. [aMHE]

Petocz, A. (1999) Freud, psychoanalysis, and symbolism. Cambridge University Press. [SB]

Pezdek, K., Finger, K. \& Hodge, D. (1997) Planting false childhood memories: The role of event plausibility. Psychological Science 8:437-41. [MCA, DHG]

Phillips, M. L., Medford, N., Senior, C., Bullmore, E. T., Suckling, J., Brammer, M. J., Andrew, C., Sierra, M., Williams, S. C. R. \& David, A. S. (2001) Depersonalization disorder: Thinking without feeling. Psychiatry Research: Neuroimaging 108:145-60. [NM]

Pintar, J. (2000) Anticipating consequences: What Bosnia has taught us about healing the wounds of war. Human Rights Review 1(2):56-66. [JP]

Plattner, B., Silvermann, M. A., Redlich, A. D., Carrion, V. G., Feucht, M., Friedrich, M. H. \& Steiner, H. (2003) Pathways to dissociation: Intrafamilial versus extrafamilial trauma in juvenile delinquents. The Journal of Nervous and Mental Disease 191:781-88. [JJF]

Pope, H. G., Jr., Hudson, J. I., Bodkin, J. A. \& Oliva, P. (1998) Questionable validity of "dissociative amnesia" in trauma victims: Evidence from prospective studies. British Journal of Psychiatry 172:210-15. [aMHE]

Pope, H. G., Oliva, P. S. \& Hudson, J. I. (1999) Repressed memories: The scientific status. In: Modern scientific evidence: The law and science of expert testimony, vol. 1, ed. D. L. Faigman, D. H. Kaye, M. J. Saks \& J. Sanders, pp. 115-15. West. [FC, JFK]

Posner, M. I. \& Fan, J. (in press) Attention as an organ system. In: Topics in integrative neuroscience: From cells to cognition, ed. J. R. Pomerantz. Cambridge University Press. [AR]

Pötzl, O. (1917) The relationship between experimentally induced dream images and indirect vision. In: Psychological Issues 2, No. 3, ed. \& trans. J. Wolff, D. Rapaport \& S. H. Annin, Monograph 7:41-120, translation of Experimentell erregte Traumbilder in ihren Beziehungen zum indirekten Sehen. Zeitschrift für die gesamte Neurologie und Psychiatrie 37:278-349. [arMHE, MS]

Powell, R. \& Boer, D. P. (1995) Did Freud misinterpret reported memories of sexual abuse as fantasies? Psychological Reports 77:563-70. [DHG]

Power, M. J., Dalgleish, T., Claudio, V., Tata, P. \& Kentish, J. (2000) The directed forgetting of emotional material. Journal of Affective Disorders $57: 147-57 . \quad$ [RJM]

Prince, M. (1910) Chapter Five. In: Subconscious phenomena, ed. H. Münsterberg, T. Ribot, P. Janet, J. Mastrow, B. Hart \& M. Prince, pp. 71-101. Gorham Press. [RGK]

(1925) Awareness, consciousness, co-consciousness and animal intelligence from the point of view of the data of abnormal psychology: A biological theory of consciousness. Pedagogical Seminary 32:166-88. [RGK]

Pyszczynski, T., Greenberg, J. \& Salomon, S. (1999) A dual-process model of defense against conscious and unconscious death-related thoughts: An extension of terror management theory. Psychological Review 106:835-45. [aMHE]

Pyszczynski, T., Greenberg, J. \& Salomon, S. (2000) Proximal and distal defense: A new perspective on unconscious motivation. Current Directions in Psychological Science 9:156-59. [aMHE] 
Rader, C. M., Kunzendorf, R. G. \& Carrabino, C. (1996) The relation of imagery vividness, absorption, reality boundaries and synesthesia to hypnotic states and traits. In: Hypnosis and imagination, ed. R. G. Kunzendorf, N. P. Spanos \& B. Wallace, pp. 99-121. Baywood. [RGK]

Raes, F., Hermans, D., de Decker, A., Eelen, P. \& Williams, J. M. G. (2003) Autobiographical memory specificity and affect-regulation: An experimental approach. Emotion 3:201-206. [DH]

Raes, F., Hermans, D., Williams, J. M. G. \& Eelen, P. (2006) Reduced autobiographical memory specificity and affect regulation. Cognition and Emotion 20:402-29. [DH]

Rassin, E. (2005) Thought suppression. Elsevier. [SN]

Raz, A. \& Buhle, J. (2006) Typologies of attentional networks. Nature Reviews. Neuroscience 7(5):367-79. [AR]

Raz, A., Fan, J. \& Posner, M. I. (2005) Hypnotic suggestion reduces conflict in the human brain. Proceedings of the National Academy of Science USA 102(28):9978-83. [AR]

Raz, A., Moreno-Iniguez, M., Martin, L. \& Zhu, H. (2006) Suggestion overrides the Stroop effect in highly hypnotizable individuals. Consciousness and Cognition. Available at: http://www.ncbi.nlm.nih.gov/entrez/query.fcgi? cmd=Retrieve\&db=Pub Med\&dopt=Citation\&_uids=16765063 [AR ]

Register, P. A. \& Kihlstrom, J. F. (1987) Hypnotic effects on hypermnesia. International Journal of Clinical and Experimental Hypnosis 35:155-70. [rMHE]

Ridderinkhof, K. R., van den Wildenberg, W. P. M., Segalowitz, S. J. \& Carter, C. S. (2004) Neurocognitive mechanisms of cognitive control: The role of the prefrontal cortex in action selection, response inhibition, performance monitoring, and reward-based learning. Brain and Cognition 56:129-40. [SB]

Roediger, H. L. \& Crowder, R. G. (1972) Instructed forgetting: Rehearsal control or retrieval inhibition (repression)? Cognitive Psychology 3:255-67. [aMHE]

Roediger, H. L. \& McDermott, K. B. (1995) Creating false memories: Remembering words not presented in lists. Journal of Experimental Psychology: Learning, Memory, and Cognition 21:803-14. [aMHE]

(1996) False perceptions of false memories. Journal of Experimental Psychology: Learning, Memory, and Cognition 22(3):814-16. [HH]

Roediger, H. L. \& Thorpe, L. A. (1978) The role of recall time in producing hypermnesia. Memory and Cognition 6:296-305. [aMHE]

Roediger, H. L., McDermott, K. B. \& Goff, L. M. (1997) Recovery of true and false memories: Paradoxical effects of repeated testing. In: Recovered memories and false memories, ed. M. A. Conway, pp. 118-149. Oxford University Press. [aMHE]

Salkovskis, P. M. \& Campbell, P. (1994) Thought suppression induces intrusion in naturally occurring negative intrusive thoughts. Behaviour Research and Therapy 32:1-8. [SN]

Salthouse, T. A., Siedlecki, K. L. \& Krueger, L. E. (2006) An individual differences analysis of memory control. Journal of Memory and Language 55:102-25. [RES]

Sand, R. (1988) Early nineteenth century anticipation of Freudian theory. International Review of Psycho-Analysis 15:465-79. [aMHE]

Sandler, J. \& Freud, A. (1985) The analysis of defense: The ego and the mechanisms of defense revisited. International Universities Press. [aMHE]

Sargant, W. \& Slater, E. (1941) Amnestic syndromes of war. Proceeding of the Royal Society of Medicine 34:757-64. [JJF]

Saunders, J. \& MacLeod, M. D. (2002) New evidence on the suggestibility of memory: The role of retrieval-induced forgetting in misinformation effects. Journal of Experimental Psychology: Applied 8:127-42. [MCA]

Schacter, D. L. (1996) Searching for memory: The brain, the mind, and the past. BasicBooks. [aMHE]

(1999) The seven sins of memory: Insights from psychology and cognitive neuroscience. American Psychologist 54:182-203. [aMHE]

(2001) Suppression of unwanted memories: Repression revisited? Lancet 357:1724-25. [JFK]

Scharnberg, M. (1993) The non-authentic nature of Freud's observations, vol. I: The seduction theory. Uppsala Studies in Education 47. Almqvist \& Wiksell International. [AE]

Schimek, J. (1987) Fact and fantasy in the seduction theory: A historical review. Journal of the American Psychoanalytic Association 35:937-65. [FC, AE]

Schimmack, U. \& Hartmann, K. (1997) Individual differences in the memory representation of emotional episodes: Exploring the cognitive processes in repression. Journal of Personality and Social Psychology 73(5):1064-79. [JMB]

Schmidt, R. E. \& Gendolla, G. H. E. (2006) Hypnagogic upheaval: The return of a suppressed thought at sleep-onset. Paper presented at the symposium, When thoughts run out of control: The vicissitudes of falling asleep (R. E. Schmidt, Chair). Conducted at the 18th Annual Convention of the Association for Psychological Science, New York, May 2006. [RES]

Schooler, J. W. \& Eich, E. (2000) Memory for emotional events. In: The Oxford Handbook of Memory, ed. E. Tulving \& F. I. M. Craik. Oxford University Press. [aMHE]
Schredl, M. (2003) Continuity between waking and dreaming: A proposal for a mathematical model. Sleep and Hypnosis 5:38-52. [MS]

Schredl, M., Dombrowe, C., Bozzer, A. \& Morlock, M. (1999) Do subliminal stimul affect dream content? Methodological issues and empirical data. Sleep and Hypnosis 1:181-85. [MS]

Schredl, M. \& Engelhardt, H. (2001) Dreaming and psychopathology: Dream recall and dream content of psychiatric inpatients. Sleep and Hypnosis 3:44-54. [MS]

Schredl, M. \& Hofmann, F. (2003) Continuity between waking activities and dream activities. Consciousness and Cognition 12:298-308. [MS]

Schredl, M. \& Montasser, A. (1996-97) Dream recall: State or trait variable? - Part I: Model, theories, methodology and trait factors; Part II: State factors, investigations and final conclusions. Imagination, Cognition and Personality 16:181-210, 239-61. [MS]

Schultz, T. M., Passmore, J. \& Yoder, C. Y. (2003) Emotional closeness with perpetrators and amnesia for child sexual abuse. Journal of Child Sexual Abuse 12:67-88. [JJF]

Sedikides, C. (1993) Assessment, enhancement, and verification determinants of the self-evaluation process. Journal of Personality and Social Psychology, 65:317-38. [CS]

(1995) Central and peripheral self-conceptions are differentially influenced by mood: Tests of the differential sensitivity hypothesis. Journal of Personality and Social Psychology 69:759-77. [CS]

Sedikides, C. \& Green, J. D. (2000) On the self-protective nature of inconsistencynegativity management: Using the person memory paradigm to examine self-referent memory. Journal of Personality and Social Psychology 79:906-22. [CS]

(2004) What I don't recall can't hurt me: Negativity versus information inconsistency as determinants of memorial self-defense. Social Cognition 22:4-29. [CS]

Sedikides, C., Green, J. D. \& Pinter, B. T. (2004) Self-protective memory. In: The self and memory, ed. D. Beike, J. Lampinen \& D. Behrend, pp. 161-79. Psychology Press. [CS]

Sedikides, C. \& Gregg, A. P. (2003) Portraits of the self. In: Sage handbook of social psychology, ed. M. A. Hogg \& J. Cooper, pp. 110-38. Sage. [CS]

Sedikides, C. \& Strube, M. J. (1997) Self-evaluation: To thine own self be good, to thine own self be sure, to thine own self be true, and to thine own self be better. In: Advances in experimental social psychology, vol. 29, ed. M. P. Zanna, pp. 209-69. Academic Press. [CS]

Shahar, G. (2001) Personality, shame, and the breakdown of social ties: The voice of quantitative depression research. Psychiatry: Interpersonal and Biological Processes 64:229-38. [GS]

(2004) Transference-countertransference: Where the (political) action is. Journal of Psychotherapy Integration 14:371-96. [GS]

(2006) Clinical action: Introduction to the special section on the action perspective in clinical psychology. Journal of Clinical Psychology 62:1053-1064. [GS]

Shane, M. S. \& Peterson, J. B. (2004a) Defensive copers show a deficit in passive avoidance learning on Newman's Go/No-go task: Implications for self-deception and socialization. Journal of Personality 72:939-65. [aMHE] (2004b) Self-induced memory distortions and the allocation of processing resources at encoding and retrieval. Cognition and Emotion 18(4):533-58. [JMB]

Shay, J. (1994) Achilles in Vietnam: Combat trauma and the undoing of character. Atheneum. [JJF]

Sheiman, J. A. (1999) Sexual abuse history with and without self-report of memory loss: Differences in psychopathology, personality, and dissociation. In: Trauma and memory, ed. L. M. Williams \& V. L. Banyard, pp. 139-48. Sage. $[\mathrm{JJF}]$

Sherman, J. W., Stroessner, S. J., Loftus, S. T. \& Deguzman, G. (1997) Stereotype suppression and recognition memory for stereotypical and nonstereotypical information. Social Cognition 15:205-15. [SN]

Shiffrin, R. M. \& Schneider, W. (1977) Controlled and automatic human information-processing. 2. Perceptual learning, automatic attending, and a general theory. Psychological Review 84 (2):127-90. [AR]

Shipherd, J. C. \& Beck, J. G. (1999) The effects of suppressing trauma-related thoughts on women with rape-related posttraumatic stress disorder. Behaviour Research and Therapy 37:99-112. [RJM, SN]

Sierra, M., Baker, D., Medford, N. \& David, A. S. (2005) Unpacking the depersonalization syndrome: An exploratory factor analysis on the Cambridge Depersonalization Scale. Psychological Medicine 35:1523-32. [NM]

Simeon, D., Knutelska, M., Nelson, D. \& Guralnik, O. (2003) Feeling unreal: A depersonalization disorder update of 117 cases. Journal of Clinical Psychiatry 64:990-97. [NM]

Simon, L., Greenberg, J. \& Brehm, J. (1995) Trivialization: The forgotten mode of dissonance reduction. Journal of Personality and Social Psychology 68:247-60. [aMHE] 
Simons, R. C. \& Hughes, C. C., eds. (1985) The culture-bound syndromes: Folk illnesses of psychiatric and anthropological interest. D. Reidel. [AR]

Sivers, H., Schooler, J. \& Freyd, J. J. (2002) Recovered memories. In: Encyclopedia of the Human Brain, vol. 4, ed. V. S. Ramachandran, pp. 169-84. Academic Press. [JJF]

Smith, A. P. R., Henson, R. N. A., Dolan, R. J. \& Rugg, M. D. (2004) fMRI correlates of the episodic retrieval of emotional contexts. Neuroimage 22:868-78. [NM]

Smith, S. M., Gleaves, D. H., Pierce, B. H., Williams, T. L., Gilliland, T. R. \& Gerkens, D. R. (2003) Eliciting and comparing false and recovered memories: An experimental approach. Applied Cognitive Psychology 17:251-79. [aMHE, JFK, SMS]

Smith, S. M. \& Vela, E. (1991) Incubated reminiscence effects. Memory and Cognition 19(2):168-76. [SMS]

Spelke, E., Hirst, W. \& Neisser, U. (1976) Skills of divided attention. Cognition $4: 215-30 . \quad[\mathrm{AR}]$

Spiegel, D. (1986) Dissociating damage. American Journal of Clinical Hypnosis 29:123-31. [RGK]

(1990) Hypnosis, dissociation, and trauma: Hidden and overt observers. In: Repression and dissociation: Implications for personality theory, psychopathology, and health, ed. J. L. Singer, pp. 471-96. University of Chicago Press. [RGK]

Stoler, L. R. (2001) Recovered and continuous memories of childhood sexual abuse: A quantitative and qualitative analysis. Doctoral Dissertation, University of Rhode Island. Dissertation Abstracts International 61(10-B), No.5582. [JJF]

Storm, B., Bjork, E. L., Bjork, R. A. \& Nestojko, J. (in press) Is retrieval success a necessary condition for retrieval-induced forgetting? Psychonomic Bulletin and Review. [MCA]

Strange, D., Gerrie, M. P. \& Garry, M. (2005) A few seemingly harmless routes to a false memory. Cognitive Processing 6:237-42. [HH]

Swets, J. A., ed. (1964) Signal detection and recognition in human observers. Wiley. [aMHE]

Tangney, J. P. (1999) The self-conscious emotions: Shame, guilt, embarrassment, and pride. In: Handbook of cognition and emotion, ed. T. Dalgleish \& M. J. Power, pp. 541-68. Wiley. [GAB]

Taylor, S. E. (1989) Positive illusions: Creative self-deception and the healthy mind. BasicBooks. [aMHE]

Taylor, S. E. \& Brown, J. (1988) Illusion and well-being: A scocial psychological perspective on mental health. Psychological Bulletin 103:193-210. [aMHE]

Tellegen, A. \& Atkinson, G. (1974) Openness to absorbing and self-altering experiences ("absorption"), a trait related to hypnotic susceptibility. Journal of Abnormal Psychology 83:268-77. [RGK]

Terr, L. (1993) Unchained memories. BasicBooks. [aMHE]

Terry, W. \& Burns, J. S. (2001) Anxiety and repression in attention and retention. Journal of General Psychology 128(4):422-32. [JMB]

Thom, D. A. \& Fenton, N. (1920) Amnesia in war cases. American Journal of Insanity 76:437-48. [JJF]

Timpanaro, S. (1976) The Freudian slip: Psychoanalysis and textual criticism, trans. K. Soper. New Left Books. [AE]

Tromp, S., Koss, M., Figueredo, A. J. \& Tharan, M. (1995) Are rape memories different? A comparison of rape, other unpleasant, and pleasant memories among employed women. Journal of Traumatic Stress 4:607-27. [JP]

Tulving, E. (1983) Elements of episodic memory. Oxford University Press. [aMHE]

(1987) Multiple memory systems and consciousness. Human Neurobiology 6:67-80. [aMHE]

Tulving, E. \& Pearlstone, Z. (1966) Availability versus accessibility of information in memory for words. Journal of Verbal Learning and Verbal Behavior 6:175-84. [aMHE]

Tulving, E. \& Schacter, D. L. (1990) Priming and human memory. Science 247:301-306. [aMHE]

Tzelgov, J. (1997) Automatic but conscious: That is how we act most of the time. In: The automaticity of everyday life, ed. R. S. Wyer Jr., pp. 217-30. Erlbaum. [GS]

Vaillant, G. E. (1998) Where do we go from here? In: Defense mechanisms in contemporary personality research, ed. P. B. Cramer \& K. Davidson. Journal of Personality [Special Issue] 66:1147-57. [aMHE]

van der Kolk, B. A. (1994) The body keeps the score: Memory and the psychobiology of posttraumatic stress. Harvard Review of Psychiatry 1:253-65. [aMHE]

van der Kolk, B. A., McFarlane, A. \& Weisaeth, L. (1996) Traumatic stress: The effects of overwhelming experiences on mind, body, and society. Guilford. [aMHE]

Veldhuis, C. B. \& Freyd, J. J. (1999) Groomed for silence, groomed for betrayal. In: Fragment by fragment: Feminist perspectives on memory and child sexual abuse, ed. M. Rivera, pp. 253-82. Gynergy Books. [JJF]
Wallon, H. (1951/1984) The psychological and sociological study of the child. In: The world of Henri Wallon, trans. D. Nicholson-Smith. J. Aaronson. (Original work published 1951). [JP]

Webster, R. (1995) Why Freud was wrong: Sin, science and psychoanalysis. HarperCollins. [AE]

Wegner, D. M. (1989) White bears and other unwanted thoughts: Suppression, obsession, and the psychology of mental control. Viking/Penguin. [aMHE, DHG, SN, SMS]

(1994) Ironic processes of mental control. Psychological Review 101:34-52. [RJM, SN, RES]

(2002) The illusion of conscious will. Bradford Books. [aMHE]

Wegner, D. M., Broome, A. \& Blumberg, S. J. (1997) Ironic effects of trying to relax under stress. Behaviour Research and Therapy 35:11-21. [SN]

Wegner, D. M. \& Erber, R. E. (1992) The hyperaccessibility of suppressed thoughts. Journal of Personality and Social Psychology 63:903-12. [SN]

Wegner, D. M., Erber, R. E. \& Zanakos, S. (1993) Ironic processes in the mental control of mood and mood-related thought. Journal of Personality and Social Psychology 65:1093-1104. [SN]

Wegner, D. M. \& Gold, D. B. (1995) Fanning old flames: Emotional and cognitive effects of suppressing thoughts of a past relationship. Journal of Personality and Social Psychology 68:782-92. [SN]

Wegner, D. M., Schneider, D. J., Carter, S. III. \& White, L. (1987) Paradoxical effects of thought suppression. Journal of Personality and Social Psychology 53:5-13. [SN]

Wegner, D. M., Wenzlaff, R. M. \& Kozak, M. (2004) Dream rebound: The return of suppressed thoughts in dreams. Psychological Science 15:232-36. [MS, RES]

Weinberger, D. A. (1990) The construct validity of the repressive coping style. In: Repression and dissociation: Implications for personality theory, psychopathology, and health, ed. J. L. Singer, pp. 337-86. University of Chicago Press. [JMB, aMHE]

Weinberger, D. G., Schwartz, G. \& Davidson, R. (1979) Low-anxious, high-anxious and repressive coping styles: Psychometric patterns and behavioral and physiological responses to stress. Journal of Abnormal Psychology 88(4):369-80. [JMB, aMHE]

Weiner, B. (1968) Motivated forgetting and the study of repression. Journal of Personality 36:213-34. [aMHE]

Weiskrantz, L., Warrington, E. K., Sanders, M. D. \& Marshall, J. (1974) Visual capacity in the hemianopic field following a restricted occipital ablation. Brain 97:709-28. [aMHE]

Wenk-Sormaz, H. (2005) Meditation can reduce habitual responding. Alternative Therapies in Health and Medicine 11(2):42-58. [AR]

Wenzlaff, R. M. \& Wegner, D. M. (2000) Thought suppression. Annual Review of Psychology 51:59-91. [SN, RES]

Wenzlaff, R. M. \& Wegner, D. M. (2000) Thought suppression. In: Annual review of psychology, vol. 51, ed. S. T. Fiske, pp. 59-91. Annual Reviews. [SN]

Werner, H. (1956) Microgenesis and aphasia. Journal of Abnormal and Social Psychology 52:347-53. [arMHE]

Wessel, I. \& Hauer, B. J. A. (2006) Retrieval-induced forgetting of autobiographical memory details. Cognition and Emotion 20:430-47. [MCA]

Wickramasekera, I. (1994) Somatic to psychological symptoms and information transfer from implicit to explicit memory: A controlled case study with predictions from the high risk model of threat perception. Dissociation 7:153-66. [DHG]

Widiger, T. \& Sankis, L. (2000) Adult psychopathology: Issues and controversies. Annual Review of Psychology 51:377-404. [DHG]

Wilhelm, S., McNally, R. J., Baer, L. \& Florin, I. (1996) Directed forgetting in obsessive-compulsive disorder. Behaviour Research and Therapy 34:633-41. [RJM]

Williams, J. M. G. \& Broadbent, K. (1986) Autobiographical memory in suicide attempters. Journal of Abnormal Psychology 95:144-49. [DH]

Williams, J. M. G., Barnhofer, T., Crane, C., Hermans, D., Raes, F., Watkins, E. \& Dalgleish, T. (in press) Autobiographical memory specificity and emotional disorder. Psychological Bulletin. [DH]

Williams, J. M. G., Watts, F. N., MacLeod, C. \& Mathews, A. (1997) Cognitive psychology and emotional disorders, 2nd edition. Wiley. [RJM]

Williams, L. M. (1994) Recall of childhood trauma: A prospective study of women's memories of child abuse. Journal of Consulting and Clinical Psychology 62:1167-76. [GAB]

(1995) Recovered memories of abuse in women with documented child sexual victimization histories. Journal of Traumatic Stress 8:649-74. [JJF]

Wright, R. (1994) The moral animal: The new science of evolutionary psychology. Pantheon. [aMHE]

Zurbriggen, E. L. (2005) Lies in a time of threat: Betrayal blindness and the 2004 U.S. presidential election. Analyses of Social Issues and Public Policy 5(1):189-96. [JJF] 